\title{
On level-set approach to motion of manifolds of arbitrary codimension
}

\author{
DEJAN SLEPČEV ${ }^{\dagger}$ \\ Department of Mathematics, University of Toronto, \\ Toronto, ON M5S 3G3, Canada
}

[Received 31 August 2002 and in revised form 22 April 2003]

\begin{abstract}
Ambrosio and Soner [1] have shown that level-set methods can be used to characterize classical, and define generalized, evolution by mean curvature of manifolds of arbitrary codimension in $\mathbb{R}^{n}$. We investigate for what (other) normal velocities, and how, the level-set methods can be used to treat motion of manifolds of arbitrary codimension by the given velocity. Two variants of the level-set approach are studied. One uses the properties of the distance function to describe the motion. In the other one, the moving manifolds are represented as a zero-level set of a solution to a parabolic differential equation. Necessary conditions and sufficient conditions for these approaches to be applicable are given. The motion of curves in $\mathbb{R}^{n}$, by a velocity that is parallel to the normal vector, is studied in greater detail and the velocities to which the level-set methods apply are partially classified. We also compare the level-set approach with the use of minimal barriers of De Giorgi, and show that for many velocities the two approaches are essentially equivalent. That in turn provides new information on applicability of barriers of De Giorgi.
\end{abstract}

\section{Introduction}

The focus of this paper is on the motion by normal velocity of manifolds of codimension greater than one embedded in $\mathbb{R}^{n}$. Such motions are encountered in models of superconductivity, medical imaging and motion of vortex filaments in fluids. Our aim is to investigate which of the motions can be treated via the level-set approach. In a sense, we investigate what motions can be described via a single parabolic partial differential equation.

The inspiration for this investigation came from the work of Ambrosio and Soner [1] on the level-set approach. Following an idea by De Giorgi, they showed that evolution by the mean curvature vector of manifolds of arbitrary codimension in $\mathbb{R}^{n}$ can be characterized by level-set methods. Geometrically, the idea is to describe the evolution of codimension $n-k>1$ manifolds using the evolution of their tubular neighborhoods. To be more precise, let $\left\{\Gamma_{t}\right\}_{t \in[0, T]}$ be a smooth family of $k$-dimensional manifolds in $\mathbb{R}^{n}$, and let $V_{k}$ be a codimension 1 velocity equal to (minus) the sum of the $k$ smallest principal curvatures at a point on a hypersurface. Ambrosio and Soner proved that the manifolds $\Gamma_{t}$ evolve by the mean curvature vector if and only if the tubes of points of fixed distance $\varepsilon$ to $\Gamma_{t}$ evolve with (outward normal) velocity less than $V_{k}$, for all $\varepsilon$ small enough. The latter property is equivalent to the distance function being a viscosity supersolution of a parabolic partial differential equation (the level-set equation that corresponds to the codimension 1 motion by velocity $V_{k}$ ). They also showed that manifold evolving by mean curvature is the zero-level set of a nonnegative function, $u$, whose level sets (for positive levels) evolve by velocity $V_{k}$, in a generalized sense. In this case $u$ is the viscosity solution of the level-set equation that corresponds

†Email: slepcev@math.toronto.edu 
to velocity $V_{k}$. The important point is that the evolution of manifolds of arbitrary codimension by the mean curvature vector can be recast in terms of the level-set approach to codimension 1 motion.

The motion (propagation, evolution, flow) of manifolds (fronts, interfaces) of codimension 1 has been studied extensively. Particularly the motion by (outward) normal velocities of the form $V(D n, n)$, where $n$ is the unit outward normal vector and $D n$ its derivative, and the velocity $V$ is continuous and nonincreasing in the $D n$ argument. One of the main difficulties in treating these motions is that the moving fronts can develop singularities and change topological type. This creates the need to introduce a notion of a weak (generalized) motion, past the singularities. One successful approach to that problem is the level-set approach, which was introduced by Osher and Sethian in [16] for numerical purposes. Viscosity solutions of the level-set equations were first used by Evans and Spruck [11] to define generalized front propagation for motion by mean curvature, and simultaneously by Chen, Giga and Goto [8] to define generalized front propagation for a large class of velocities. Ishii and Souganidis [14] have extended the level-set approach to all the velocities, $V$, described at the beginning of this paragraph.

Let us briefly recall the level-set approach. At each time the front, $\Upsilon_{t}$, is described as the zerolevel set of a function $u$, while the interior of the front, $\Omega_{t}$, is the set where $u$ is less than zero. When the function $u$ is differentiable and $|D u| \neq 0$ on the front then

$$
n=\frac{D u}{|D u|}, \quad D n=\frac{1}{|D u|}\left(I-\frac{D u \otimes D u}{|D u|^{2}}\right) D^{2} u, \quad V=\frac{-u_{t}}{|D u|}
$$

For a symmetric matrix $X$ and a nonzero vector $p$ we define

$$
F(X, p):=|p| V\left(\frac{1}{|p|}(I-\hat{p} \otimes \hat{p}) X(I-\hat{p} \otimes \hat{p}), \hat{p}\right)
$$

where $\hat{p}=p /|p|$. The equality $V=-u_{t} /|D u|$ then reads $u_{t}+F\left(D^{2} u, D u\right)=0$ on $\Gamma_{t}$. If we require that all the level sets of $u$ propagate by velocity $V$ then we get an equation on the whole space. That motivates the following definition.

We say that the family $\left\{\left(\Omega_{t}, \Upsilon_{t}\right)\right\}_{t \in[0, T]}$ is the generalized evolution by velocity $V$ if $\Omega_{t}=\{x$ : $u(x, t)<0\}$ and $\Upsilon_{t}=\{x: u(x, t)=0\}$, where $u$ is the unique viscosity solution of the level-set equation

$$
\begin{aligned}
& u_{t}+F\left(D^{2} u, D u\right)=0, \\
& u(x, 0)=u_{0}(x)
\end{aligned}
$$

Here $u_{0}$ is any uniformly continuous function, negative in $\Omega_{0}$, zero on $\Upsilon_{0}$, and positive on the complement of $\Omega_{0} \cup \Upsilon_{0}$.

Another, similar way to define generalized motion is by using the properties of the signed distance function to the front. This approach was developed by Soner [17], and further investigated by Barles, Soner and Souganidis in [3], and Ishii and Souganidis in [14]. Let $O_{t}$ be the set enclosed by the front at time $t$, and $\Gamma_{t}=\partial O_{t}$ the front. The signed distance function is defined by

$$
d_{ \pm}(x, t):= \begin{cases}-\operatorname{dist}\left(x, \Gamma_{t}\right) & \text { if } x \in O_{t} \\ \operatorname{dist}\left(x, \Gamma_{t}\right) & \text { otherwise }\end{cases}
$$


The family $\left\{\left(O_{t}, \Gamma_{t}\right)\right\}$ is said to be the generalized evolution by velocity $V$ if

$$
\begin{aligned}
& \frac{\partial\left(d_{ \pm} \wedge 0\right)}{\partial t}+F\left(D^{2}\left(d_{ \pm} \wedge 0\right), D\left(d_{ \pm} \wedge 0\right)\right) \leqslant 0, \\
& \frac{\partial\left(d_{ \pm} \vee 0\right)}{\partial t}+F\left(D^{2}\left(d_{ \pm} \vee 0\right), D\left(d_{ \pm} \vee 0\right)\right) \geqslant 0,
\end{aligned}
$$

in the viscosity sense. Here $a \wedge b=\min \{a, b\}$ and $a \vee b=\max \{a, b\}$.

These two approaches are in large part equivalent; the difference being in how they represent the evolution when it is not unique, for example, the evolution by the mean curvature of the union of two coordinate axes in $\mathbb{R}^{2}$. In such cases, there is more than one front propagating by the given velocity in the sense of the definition via the distance function, while there is just one generalized evolution that comes from the level-set equation, since it has a unique solution. However, the set $\Upsilon_{t}$ is no longer the boundary of $\Omega_{t}$; it itself has a nonzero measure. This phenomenon is called front fattening. The precise relationship between two approaches is the following: Let $\left\{\left(\Omega_{t}, \Upsilon_{t}\right)\right\}_{t}$ be the generalized evolution of the front by velocity $V$ using the former definition. Then $\left\{\left(\Omega_{t}, \partial \Omega_{t}\right)\right\}_{t}$ is the minimal (in the sense of the set inclusion of the front interiors) and $\left\{\left(\operatorname{int}\left(\Omega_{t} \cup \Upsilon_{t}\right), \partial\left(\Omega_{t} \cup \Upsilon_{t}\right)\right)\right\}_{t}$ the maximal generalized evolution (starting from $\left(\Omega_{0}, \Upsilon_{0}\right)$ ) in the sense of the latter definition.

The results of Ambrosio and Soner can now be restated as follows: Let $F_{k}$ be the function that by the equation (1.1) corresponds to the velocity $V_{k}$. A smooth family of $k$-dimensional manifolds, $\Gamma_{t}$, in $\mathbb{R}^{n}$ evolves by the mean curvature vector if and only if the distance function to $\Gamma_{t}$ satisfies

$$
d_{t}+F_{k}\left(D^{2} d, D d\right) \geqslant 0
$$

in the viscosity sense. Also, manifolds $\Gamma_{t}$ move by the mean curvature vector if and only if $\Gamma_{t}=$ $\{x: u(x, t)=0\}$ where $u$ is the viscosity solution of the level-set equation 1.2. In this case the initial condition, $u_{0}$, is any uniformly continuous function equal to 0 on $\Gamma_{0}$ and positive on the complement of $\Gamma_{0}$. Both of these characterizations can then be used to define generalized evolution by mean curvature vector. Note, however, that there are important differences in the way the level-set equation is used to describe codimension 1 and codimension greater than 1 motions. In particular, for codimension 1 motions, a generic level set is a hypersurface evolving with the given velocity, while only the zero-level set evolves with the given codimension greater than 1 velocity. Also, no set is enclosed by a moving manifold of codimension greater than 1 , and there is no geometric comparison principle that the moving manifolds satisfy directly.

Our objective is to study if characterizations similar to the ones described above can be obtained for general codimension $n-k$ velocities. We say that a codimension 1 velocity $V$ gives a distance representation to a codimension $n-k$ velocity $v$ if for every smooth family of $k$-dimensional manifolds in $\mathbb{R}^{n},\left\{\Gamma_{t}\right\}_{t \in[0, T]}$, the following holds: The manifolds $\Gamma_{t}$ evolve by velocity $v$ if and only if the distance function to $\Gamma_{t}$ satisfies

$$
d_{t}+F\left(D^{2} d, D d\right) \geqslant 0
$$

in the viscosity sense. Here $F$ is again given by (1.1). In a similar fashion, we say that a codimension 1 velocity $V$ surrounds a codimension $n-k$ velocity $v$ if manifolds moving with velocity $v$ can be characterized as the zero-level set of the solution of the level-set equation (1.2). Our main goal is to find out what codimension $n-k$ velocities $v$ have a distance representation (or can be surrounded), as well as how to find the "best" codimension 1 velocity $V$ that gives a distance representation to $v$. 
The paper is organized as follows: In Section 2 we review the level-set approach to motion of manifolds of codimension 1. In addition, we show that for any smooth codimension 1 velocity, $V(D n, n)$, nonincreasing in the $D n$ argument, and any compact closed smooth hypersurface without boundary, there exists a unique short time classical evolution by velocity $V$. In Section 3 we establish some properties of the distance function in a tubular neighborhood of a codimension $n-k$ manifold $\Gamma$ embedded in $\mathbb{R}^{n}$. In particular, we compute $D d$ and $D^{2} d$ near $\Gamma$.

In Section 4 we begin investigating the motion by codimension greater than 1 velocities. We define notions we use (distance representation, surrounding etc.), establish relationships among them, and find some sufficient conditions and a necessary condition that the velocities $v$ and $V$ have to satisfy, in order for $V$ to give a distance representation to $v$ and for $V$ to surround $v$. The techniques we use here are inspired by the work of Ambrosio and Soner on motion by mean curvature vector. Our approach is, however, more direct and hence yields simpler proofs.

Completely classifying which velocities have a distance representation appears to be a difficult task, as even the special case of motion of curves in $\mathbb{R}^{n}$ has a rich structure. We investigate the motion of curves in $\mathbb{R}^{n}$ in Section 5. For motion of curves by a velocity that is parallel to the normal vector we are able to find conditions that are very close to being both necessary and sufficient for a velocity $V$ to give a distance representation to $v$. We then discuss when a given velocity $v$ has a distance representation, and find (the "best") velocity $V$ that gives the distance representation. After that, we partially classify the velocities that have a distance representation. In particular we prove that the only velocities of the form $f(k) \vec{n}$ (where $k$ is the curvature and $\vec{n}$ the normal vector) that have a distance representation are constant multiples of the curvature vector (i.e. $v=C k \vec{n}$ ). For the velocities of the form $f(k, \vec{t}) \vec{n}$ (where $\vec{t}$ is the tangent vector) the results are more intriguing. Under a technical condition, we show that velocities of that form have a distance representation if $f(k, \vec{t})=g(\vec{t}) k$, where $g$ is a nonnegative even function whose set of zeroes satisfies an interesting, geometric condition related to convexity (in $S^{n-1}$ ). Let us just mention that, for example, the velocity with $g(\vec{t})=1-|\vec{e} \cdot \vec{t}|^{2}$, where $\vec{e}$ is an arbitrary unit vector, has a distance representation, while the velocity with $g(\vec{t})=|\vec{e} \cdot \vec{t}|^{2}$ does not.

In Section 6 we turn our attention to the barriers of De Giorgi, which offer an abstract way to define generalized evolution by a given velocity. For motion of fronts of codimension 1 this approach was developed by De Giorgi, Bellettini, Novaga and Paolini (see [5] and references therein). They proved that the barriers give the same information about the front as the level-set approach. De Giorgi conjectured that for the motion by mean curvature vector in any codimension the levelset approach and the barriers approach are essentially equivalent as well. A part of the conjecture was proven by Ambrosio and Soner in [1], while the complete conjecture was proven by Bellettini and Novaga in [6]. The main topic of Section 6 is comparing the level-set approach and the barriers approach. We concentrate on the motion of curves in $\mathbb{R}^{n}$, and prove that for a large class of velocities the two approaches are equivalent. We also show that for many velocities for which the level-set approach is not applicable, neither is the barriers approach. At the end we offer a simple proof of De Giorgi's conjecture.

\section{Motion of manifolds of codimension 1}

In this section we recall the level-set approach to motion (propagation, evolution, flow) of manifolds (fronts, interfaces) of codimension 1 in $\mathbb{R}^{n}$. The normal velocities that we consider have the form $V(D n, n)$, where $n$ is the outward normal vector to the front, and $D n$ its derivative. We furthermore require that $V$ is nonincreasing in the $D n$ argument. To consider evolution (flow) of a manifold by 
a given velocity we first define the classical notion of evolution (flow, propagation) by given normal velocity:

DEFINITION 2.1 Let $\Upsilon$ be a compact, connected, $n-1$-dimensional, $C^{2}$ manifold without boundary and let $\psi \in C^{2}\left(\Upsilon \times[0, T], \mathbb{R}^{n}\right)$ be an embedding for every fixed $t \in[0, T]$. Let $\Upsilon_{t}:=\psi(\Upsilon \times\{t\})$ and let $\Omega_{t}$ be the bounded component of $\mathbb{R}^{n} \backslash \Upsilon_{t}$. We say that $\left\{\left(\Omega_{t}, \Upsilon_{t}\right)\right\}_{t \in[0, T]}$ is the classical evolution (flow) of $\left(\Omega_{0}, \Upsilon_{0}\right)$ by velocity $V$ if

$$
\frac{\partial \psi}{\partial t}(x, t) \cdot n=V(D n, n)
$$

where $n$ is the outward normal vector to $\Upsilon_{t}$ at $\psi(x, t)$.

We say that $\left\{\left(\Omega_{t}, \Upsilon_{t}\right)\right\}_{t \in[0, T]}$ is a classical subflow (resp. superflow) by velocity $V$ if the equality in the condition above is replaced by $\leqslant($ resp. $\geqslant)$.

However, the classical motion of a front often exists for only a short time, since the front can develop singularities. We use the viscosity solutions of level-set equations for the given motion to define generalized front propagation. More precisely, we use the definition of viscosity solutions introduced by Ishii and Souganidis in [14] that allows us to consider velocities with arbitrary growth of the curvature tensor.

DEFINITION 2.2 Let $\Omega_{0}$ be a bounded open set and $\Upsilon_{0}=\partial \Omega_{0}$ be the front at time $t=0$. Let $u_{0}$ be a uniformly continuous function such that $\Upsilon_{0}=\left\{x \in \mathbb{R}^{n}: u_{0}(x)=0\right\}$ and $\Omega_{0}=\left\{x: u_{0}(x)<0\right\}$. We say that $\left\{\left(\Omega_{t}, \Upsilon_{t}\right)\right\}_{t \in[0, T]}$ is the generalized evolution of $\left(\Omega_{0}, \Upsilon_{0}\right)$ by velocity $V$ for $t \in[0, T)$ if $\Upsilon_{t}:=\{x: u(x, t)=0\}$ and $\Omega_{t}:=\{x: u(x, t)<0\}$, where $u$ is the viscosity solution (as defined in [14]) of the level-set equation:

$$
\begin{array}{ll}
u_{t}+F\left(D^{2} u, D u\right)=0 & \text { on } \mathbb{R}^{n} \times(0, T), \\
u(x, 0)=u_{0}(x) & \text { for all } x \in \mathbb{R}^{n}
\end{array}
$$

with $F$ given by (1.1).

Since the exact definition of viscosity solutions in [14] is rather technical, we refer the reader to the Appendix for details. It is, however, important to keep in mind that solutions of [14] are the same as the standard viscosity solutions of level-set equations (see, for example, [8]) when $F^{*}(0,0)=$ $F_{*}(0,0)=0$. Here $F^{*}$ (resp. $F_{*}$ ) is the upper (resp. lower) semicontinuous envelope of $F$. Ishii and Souganidis have shown that the equation 2.1) has a unique uniformly continuous viscosity solution (for any $T \in(0, \infty]$ ) and that the definition above does not depend on the choice of the function $u_{0}$.

\subsection{Properties of generalized evolution}

We now list some important properties of generalized evolution and viscosity solutions, that were either (in this generality) proven in [14] or have simple proofs. In all these properties (where relevant) $\left\{\left(\Omega_{t}, \Upsilon_{t}\right)\right\}_{t \in[0, T]}$ is assumed to be a generalized evolution by a velocity $V$.

(P1) Invariance under nondecreasing transformations. Or in other words, invariance under relabeling of level sets. Let $\rho$ be a nondecreasing function. If $u$ is a viscosity supersolution (resp. subsolution) of (2.1) then so is $\rho(u)_{*}$ (resp. $\left.\rho(u)^{*}\right)$. If $\rho$ is continuous and $u$ is a uniformly continuous solution of 2.1 , then $\rho(u)$ is a continuous solution, with initial data $\rho\left(u_{0}\right)$. 
(P2) Comparison of viscosity solutions. If $u$ is an (upper semicontinuous) subsolution of [2.1], $v$ a (lower semicontinuous) supersolution of 2.1$)$, and $u(\cdot, 0) \leqslant v(\cdot, 0)$ then $u(\cdot, t) \leqslant v(\cdot, t)$ for all times $t>0$.

(P3) Comparison of evolving fronts. Let $\left\{\left(\Omega_{t}^{\prime}, \Upsilon_{t}^{\prime}\right)\right\}_{t \in[0, T]}$ be a generalized evolution by velocity $V$. If $\Omega_{0} \subseteq \Omega_{0}^{\prime}$ then $\Omega_{t} \subseteq \Omega_{t}^{\prime}$ and $\Upsilon_{t} \subseteq \Omega_{t}^{\prime} \cup \Upsilon_{t}^{\prime}$ for all $t>0$.

(P4) Comparison of evolutions with different velocities. Let $V_{2}$ be a velocity such that $V_{2} \geqslant V$, and $\left\{\left(\Omega_{t}^{\prime}, \Upsilon_{t}^{\prime}\right)\right\}_{t \in[0, T]}$ the generalized evolution of $\left(\Omega_{0}, \Upsilon_{0}\right)$ by velocity $V_{2}$. Then $\Omega_{t} \subseteq \Omega_{t}^{\prime}$ and $\Upsilon_{t} \subset \Omega_{t}^{\prime} \cup \Upsilon_{t}^{\prime}$ for all $t \in[0, T]$.

(P5) Agreement with classical evolution I. If $\Upsilon_{t}$ are $C^{2}$ manifolds for all $t \in[0, T]$ then $\left\{\left(\Omega_{t}, \Upsilon_{t}\right)\right\}_{t \in[0, T]}$ is a classical evolution by velocity $V$.

(P6) Agreement with classical evolution II. Let $\left\{\left(\Omega_{t}^{\prime}, \Upsilon_{t}^{\prime}\right)\right\}_{t \in[0, T]}$ be a classical evolution of $\Upsilon_{0}$ by velocity $V$. Then $\Upsilon_{t}^{\prime}=\Upsilon_{t}$ for all $t \in[0, T]$.

(P7) Comparison with sub- and superflows. Let $\left\{\left(\Omega_{t}^{\prime}, \Upsilon_{t}^{\prime}\right)\right\}_{t \in[0, T]}$ be a (classical) superflow (or subflow) by velocity $V$. If $\Omega_{0} \cup \Upsilon_{0} \subset \Omega_{0}^{\prime}$ (resp. $\Omega_{0}^{\prime} \cup \Upsilon_{0}^{\prime} \subset \Omega_{0}$ ) then $\Omega_{t} \cup \Upsilon_{t} \subset \Omega_{t}^{\prime}$ (resp. $\Omega_{t}^{\prime} \cup \Upsilon_{t}^{\prime} \subset \Omega_{t}$ ) for all $t \in[0, T]$.

\subsection{Short time existence of classical evolutions}

In addition to generalized evolution, we need the short time existence of classical evolutions by smooth velocities $V(D n, n)$ that are nonincreasing in the $D n$ argument. There are, of course, a number of results on short time existence of classical evolutions. Giga and Goto have shown in [12] that if the velocity $V$ is strictly decreasing (in a uniform way) in the $D n$ argument and the initial manifold is smooth, then there exists a short time smooth evolution. Here we show that the classical evolutions (recall that we require them to be only $C^{2}$ ) exist for short time even when $V$ is only nonincreasing in the $D n$ argument. The main tool that we use are the results by Lions and Souganidis in [15]. The following lemma is a corollary of Theorem V.1 from their paper.

LEMma 2.3 Let $G: \operatorname{Sym}(n) \times \mathbb{R} \rightarrow \mathbb{R}$ be a smooth, degenerate elliptic function such that $\|G\|_{C^{4}\left(K \times \mathbb{R}^{n}\right)}$ is bounded for every compact set $K \subset \operatorname{Sym}(n)$. Let $u_{0} \in C^{6}\left(\mathbb{R}^{n}\right)$ with $\left\|u_{0}\right\|_{C^{6}}$ finite. Then there exists a time $t_{0}>0$ such that the Cauchy problem

$$
\begin{array}{ll}
u_{t}+G\left(D^{2} u, D u\right)=0 & \text { on } \mathbb{R}^{n} \times\left(0, t_{0}\right), \\
u(x, 0)=u_{0}(x) & \text { for all } x \in \mathbb{R}^{n},
\end{array}
$$

has a $C^{2}$ solution.

We are now ready to prove the short time existence of classical evolutions:

THEOREM 2.4 Let $V(D n, n)$ be a smooth codimension 1 velocity (nonincreasing in the $D n$ argument). Let $\Omega_{0}$ be a bounded open set and $\Upsilon_{0}=\partial \Omega_{0}$ a $C^{6}$ manifold in $\mathbb{R}^{n}$. Then for some time $t_{0}>0$ there exists a unique classical evolution, $\left\{\left(\Omega_{t}, \Upsilon_{t}\right)\right\}_{t \in\left[0, t_{0}\right)}$, by velocity $V$.

Proof. Let $V, \Omega_{0}$, and $\Upsilon_{0}$ be as above and let $d_{0}(x):=d_{ \pm}\left(x, \Upsilon_{0}\right)$ (negative in $\left.\Omega_{0}\right)$. We can assume that $0 \in \Omega_{0}$. Let $M:=\operatorname{diam} \Omega_{0}$. There exists $\sigma \in(0,1)$ such that $d_{0}$ is a $C^{6}$ function on the set where $\left|d_{0}\right|<4 \sigma$. Let $\eta_{1} \in C^{\infty}(\mathbb{R},[-1,1])$ be a nonincreasing function such that $\eta_{1}(s)=-2 \sigma$ if $s \leqslant-2 \sigma, \eta_{1}(s)=s$ if $|s|<\sigma$, and $\eta_{1}(s)=2 \sigma$ if $s \geqslant 2 \sigma$. Let $\eta_{2} \in C^{\infty}([0, \infty),[0,1])$ be such that $\eta_{2}(s)=1$ for $s \in[1 / 2,2]$ and $\eta_{2}(s)=0$ for $s \in[0,1 / 4] \cup[4, \infty)$. Let $\eta_{3} \in C^{\infty}(\mathbb{R},[0,1])$ 
be a nonincreasing function such that $\eta_{3}(s)=1$ if $s<3 M$ and $\eta_{3}(s)=0$ if $s>3 M+1$. For a symmetric matrix $X$ and vector $p$ we define

$$
G(X, p):=\eta_{2}(|p|) F(X, p) .
$$

Note that $G$ is smooth and degenerate elliptic. Consider the Cauchy problem

$$
\begin{aligned}
& u_{t}+G\left(D^{2} u, D u\right)=0, \\
& u(x, 0)=\eta_{1}\left(d_{0}(x)\right) \eta_{3}(|x|) .
\end{aligned}
$$

By Lemma 2.3 this equation has a $C^{2}$ solution, $u$, for some time $t_{0}>0$. Let $\Omega_{t}:=\{x: u(x, t)<0\}$ and $\Upsilon_{t}:=\partial \Omega_{t}$. By making $t_{0}$ smaller if necessary we can assume that diam $\Omega_{t}<2 M$ for every $t \in\left[0, t_{0}\right)$, and if $|u(x)|<\sigma / 2$ and $x<2 M$ then $|D u|(x, t) \in(1 / 2,2)$. It is then easy to check that $\left\{\left(\Omega_{t}, \Upsilon_{t}\right)\right\}_{t \in\left[0, t_{0}\right)}$ is a classical evolution by velocity $V$. Let us show that $\left\{\left(\Omega_{t}, \Upsilon_{t}\right)\right\}_{t \in\left[0, t_{0}\right)}$ is a unique classical evolution for time $t \in\left[0, t_{0}\right)$. Assume that there exists another classical evolution $\left\{\left(\Omega_{t}^{\prime}, \Upsilon_{t}^{\prime}\right)\right\}_{t \in\left[0, t_{1}\right)}$ for some time $t_{1}>0$. Then there exists a time $t^{\prime}$ and a point $x^{\prime} \in \Upsilon_{t^{\prime}}^{\prime}$ such that $u\left(x^{\prime}, t^{\prime}\right) \neq 0$. Assume that $u\left(x^{\prime}, t^{\prime}\right)<0$. Let $a:=\frac{1}{4}\left(-\sigma \vee u\left(x^{\prime}, t^{\prime}\right)\right)$. Note that the level sets $\{u(\cdot, t)=a\}$ move with velocity $V$ in the classical sense. Therefore, by comparison, $\Upsilon_{t^{\prime}}^{\prime} \subset$ $\left\{u\left(\cdot, t^{\prime}\right) \geqslant a\right\}$, which is in contradiction with the fact that $u\left(x^{\prime}, t^{\prime}\right)<a$. The case $u\left(x^{\prime}, t^{\prime}\right)>0$ is analogous.

\section{Geometric preliminaries}

Let $\Gamma$ be a compact, $k$-dimensional, $C^{2}$ manifold without boundary, embedded in $\mathbb{R}^{n}$. By $T_{x} \Gamma$ we denote the tangent space to $\Gamma$ at $x$, and by $N_{x} \Gamma$ its orthogonal complement (in $\mathbb{R}^{n}$ ). To describe the local geometry of $\Gamma$ we use the shape operator $S \Gamma: N_{x} \Gamma \times T_{x} \Gamma \rightarrow T_{x} \Gamma$, defined by

$$
S_{p} \Gamma(v):=-\Pi_{T}\left(\nabla_{v} \tilde{p}\right),
$$

where $\tilde{p}$ is a local extension of $p \in N_{x} \Gamma$ such that $\tilde{p}(y)$ is orthogonal to $\Gamma$ when $y \in \Gamma$, and $\Pi_{T}$ is the orthogonal projection to $T$. If the manifold being considered is known from the context, we write $S$ instead of $S \Gamma$. Note that $S$ contains the same information as the second fundamental form $B$, since

$$
S_{p}(x) \cdot y=B(x, y) \cdot p .
$$

For a point $x$ in $\mathbb{R}^{n}$ let $d(x)$ be its distance from $\Gamma$. By $\Gamma+a$ we denote the set of points in $\mathbb{R}^{n}$ whose distance to $\Gamma$ is less than $a$. Since $\Gamma$ is a $C^{2}$ submanifold of $\mathbb{R}^{n}$ it has a tubular neighborhood (see, for example, [13]). Therefore there exists $\sigma_{1}>0$ such that for every point $x$ in $\Gamma+\sigma_{1}$, there exists a unique point on $\Gamma$ closest to $x$. Let us denote it by $\pi_{\Gamma}(x)$.

Lemma 3.1 There exists $\sigma>0$ such that for all $x_{0} \in \Gamma$, every unit vector $p \in N_{x_{0}} \Gamma$ and every $v \in T_{x_{0}} \Gamma$ the following holds:

1. $D^{2} d\left(x_{0}+t p\right)$ is a nonincreasing function of $t$ (with values in the set of symmetric matrices) for $t \in(0, \sigma)$ and

2. $\lim _{t \rightarrow 0^{+}} D^{2} d\left(x_{0}+t p\right) v=-S_{p} v$. 
Proof. We can assume that $x_{0}=0, T_{0} \Gamma=\operatorname{span}\left\{e_{1}, \ldots, e_{k}\right\}$, and $p=e_{n}$, where $\left[e_{1}, \ldots, e_{n}\right]$ are the coordinate unit vectors in $\mathbb{R}^{n}$. Set

$$
\sigma:=\min \left\{\sigma_{1}, 1 /\left(1+\max \left\{\left\|S_{w}\right\|: x \in \Gamma, w \in N_{x} \Gamma,|w|=1\right\}\right)\right\} .
$$

Let $\pi_{k}$ be the orthogonal projection from $\Gamma$ to $T_{0} \Gamma$. When restricted to a neighborhood of 0 it is a bijection (onto a neighborhood, $V$, of 0 in $T_{0} \Gamma$ ). Let $\psi$ be the inverse of the restriction. Then

$$
\psi\left(x_{1}, \ldots, x_{k}\right)=\left(x_{1}, \ldots, x_{k}, \psi^{k+1}\left(x_{1}, \ldots, x_{k}\right), \ldots, \psi^{n}\left(x_{1}, \ldots, x_{k}\right)\right) .
$$

Let $\left[n_{1}, \ldots, n_{n-k}\right]$ be an orthonormal frame on $N \Gamma$ for points in $\psi(V)$ such that $\left[n_{1}, \ldots, n_{n-k}\right]=$ $\left[e_{k+1}, \ldots, e_{n}\right]$ at $x=0$. Let us extend it to $V_{2}:=\pi_{\Gamma}^{-1}(\psi(V))$ by setting $n_{i}(x):=n_{i}\left(\pi_{\Gamma}(x)\right)$ for $i=1, \ldots, n-k$.

We now introduce new coordinates on $V_{2}$. Let $\varphi: V_{2} \rightarrow \mathbb{R}^{n}$ be defined by

$$
\varphi\left(x_{1}, \ldots, x_{k}, t_{1}, \ldots, t_{n-k}\right):=\psi\left(x_{1}, \ldots, x_{k}\right)+\sum_{j=1}^{n-k} t_{j} n_{j}\left(x_{1}, \ldots, x_{k}\right) .
$$

Note that $\varphi$ is a differentiable bijection between $V_{2}$ and $\varphi\left(V_{2}\right)$. Let us compute $D \varphi\left(\varphi^{-1}(t p)\right)$. We have

$$
\begin{aligned}
D \varphi & =\left[\frac{\partial \psi}{\partial x_{1}}+t \frac{\partial n_{n-k}}{\partial x_{1}}, \ldots, \frac{\partial \psi}{\partial x_{k}}+t \frac{\partial n_{n-k}}{\partial x_{k}}, n_{1}, \ldots, n_{n-k}\right] \\
& =\left[\begin{array}{cc}
I_{k}-t S_{p} & 0 \\
0 & I_{n-k}
\end{array}\right] .
\end{aligned}
$$

Note that the choice of $\sigma$ ensures that the matrix $I-t S_{p}$ is invertible. We now compute

$$
\begin{aligned}
D d \circ \varphi & =\frac{t_{1} n_{1}+\cdots+t_{k} n_{k}}{\sqrt{t_{1}^{2}+\cdots+t_{k}^{2}}} \\
D(D d \circ \varphi)\left(\varphi^{-1}(t p)\right) & =\left[\frac{\partial n_{n-k}}{\partial x_{1}}, \ldots, \frac{\partial n_{n-k}}{\partial x_{k}}, \frac{n_{1}}{t}, \ldots, \frac{n_{n-k-1}}{t}, 0\right] \\
& =\left[\begin{array}{ccc}
-S_{p} & 0 & 0 \\
0 & \frac{1}{t} I_{n-k-1} & 0 \\
0 & 0 & 0
\end{array}\right] .
\end{aligned}
$$

Using 3.2, we now obtain

$$
\begin{aligned}
D^{2} d(t p)=D\left((D d \circ \varphi) \circ \varphi^{-1}\right)(t p) & =\left[\begin{array}{ccc}
-S_{p} & 0 & 0 \\
0 & \frac{1}{t} I_{n-k-1} & 0 \\
0 & 0 & 0
\end{array}\right]\left[\begin{array}{cc}
\left(I_{k}-t S_{p}\right)^{-1} & 0 \\
0 & I_{n-k}
\end{array}\right] \\
& =\left[\begin{array}{ccc}
-S_{p}\left(I_{k}-t S_{p}\right)^{-1} & 0 & 0 \\
0 & \frac{1}{t} I_{n-k-1} & 0 \\
0 & 0 & 0
\end{array}\right] .
\end{aligned}
$$


Note that the matrix $A(t):=D^{2} d(t p)$ satisfies the matrix Riccati equation

$$
\frac{d A}{d t}=-A^{2}
$$

Therefore $D^{2} d(t p)$ is nonincreasing for $t \in(0, \sigma)$. Since $\left(I-t S_{p}\right)^{-1}$ approaches the identity matrix as $t$ approaches 0 , the second claim of the lemma follows from (3.3) as well.

REMARKS. 1. Claim 2 of the lemma proves the conjecture that Ambrosio and Soner made on page 707 of [1].

2. The matrix $D^{2} d(t p)$ has the form 3.3 under the assumption that $T_{0} \Gamma=\operatorname{span}\left\{e_{1}, \ldots, e_{k}\right\}$ and $p=e_{n}$. In general, for $x \in \Gamma$ and $p \in N_{x} \Gamma$, let $\left[t_{1}, \ldots, t_{k}\right]$ be an orthonormal basis of $T_{x} \Gamma$ and $\left[t_{k+1}, \ldots, t_{n}\right]$ an orthonormal basis of $N_{x} \Gamma$, where $t_{n}=p$. Let $R$ be the matrix $\left[t_{1}, \ldots, t_{n}\right]$. Then

$$
D^{2} d(t p)=R\left[\begin{array}{ccc}
-S_{p}\left(I_{k}-t S_{p}\right)^{-1} & 0 & 0 \\
0 & \frac{1}{t} I_{n-k-1} & 0 \\
0 & 0 & 0
\end{array}\right] R^{-1} .
$$

Here $S_{p}$ is the matrix of the shape operator in $\left[t_{1}, \ldots, t_{k}\right]$ coordinates.

The following lemma is partly a corollary of the previous one.

Lemma 3.2 Let $\psi \in C^{2}\left(\Gamma \times[0, T], \mathbb{R}^{N}\right)$ be an embedding for every fixed $t \in[0, T]$. Let $\Gamma_{t}:=$ $\psi(\Gamma \times\{t\})$ and $d(x, t):=\operatorname{dist}\left(x, \Gamma_{t}\right)$. Then

$$
\sigma:=1 / \max \left\{2\left\|S_{p}\right\|: x \in \Gamma, t \in[0, T], p \in N_{\psi(x, t)} \Gamma_{t},|p|=1\right\}
$$

is a positive number and the function $d(x, t)$ is twice differentiable on the set $A:=\{(x, t) \in$ $\left.\mathbb{R}^{n} \times[0, T]: d(x, t) \in(0, \sigma)\right\}$. Furthermore, if for any $(z, t) \in A$ we denote by $\Pi(z, t)$ the closest point to $z$ on $\Gamma_{t}$, then

$$
\frac{\partial d}{\partial t}(z, t)=-p \cdot \frac{\partial \psi}{\partial t}\left(\psi(\cdot, t)^{-1}(\Pi(z, t)), t\right),
$$

where $p:=(z-\Pi(z, t)) /|z-\Pi(z, t)|$.

Proof. The proof of the first part of the lemma is elementary, so we only prove the second part. Fix $(z, t) \in A$. Let $y:=\Pi(z, t)$ and $X(s):=\psi(\cdot, s)^{-1}(\Pi(z, s))$. Note that $\Pi$ and $X$ are differentiable functions. Also note that $d(z, t)=|z-\Pi(z, t)|$. We compute:

$$
\begin{aligned}
\frac{\partial d}{\partial t}(z, t) & =-\frac{z-\Pi(z, t)}{|z-\Pi(z, t)|} \cdot \frac{\partial \Pi}{\partial t}(z, t) \\
& =-p \cdot \frac{d}{d t} \psi(X(t), t) \\
& =-p \cdot\left(D \psi(X(t), t) X^{\prime}(t)+\frac{\partial \psi}{\partial t}(X(t), t)\right) \\
& =-p \cdot \frac{\partial \psi}{\partial t}(X(t), t)
\end{aligned}
$$

since $p \in N_{y} \Gamma_{t}$ and $D \psi X^{\prime}(t) \in T_{y} \Gamma_{t}$. 


\section{Motion of manifolds of codimension greater than 1}

We consider the motion of manifolds of codimension $n-k>1$ with normal velocity $v$ that depends on the tangent space to the manifold, $T$, and the second order properties of the manifold described by the shape operator $S$.

Definition 4.1 Let $\Gamma$ be a $k$-dimensional $C^{2}$ manifold without boundary, let $\psi \in C^{2}(\Gamma \times$ $\left.[0, T], \mathbb{R}^{n}\right)$ be an embedding for every fixed $t \in[0, T]$, and let $\Gamma_{t}:=\psi(\Gamma \times\{t\})$. We say that the manifolds $\Gamma_{t}$ move (evolve) with normal velocity $v\left(S \Gamma_{t}, T \Gamma_{t}\right)$, where $v\left(S \Gamma_{t}, T \Gamma_{t}\right)$ is orthogonal to $\Gamma_{t}$, if for all $x \in \Gamma$ and $t \in[0, T]$,

$$
\pi_{N_{y} \Gamma_{t}}\left(\frac{\partial \psi}{\partial t}(x, t)\right)=v\left(S \Gamma_{t}, T_{y} \Gamma_{t}\right) .
$$

Here $\pi_{N_{y} \Gamma_{t}}$ is the orthogonal projection of $\mathbb{R}^{n}$ onto the normal space to $\Gamma_{t}$ at $y=\psi(x, t)$.

Here are some examples of velocities as above,

1. Motion by ( $k$ times) mean curvature vector:

$$
v(S, T):=\sum_{i=1}^{n-k}\left(\operatorname{trace} S_{n_{i}}\right) n_{i},
$$

where $\left[n_{1}, \ldots, n_{n-k}\right]$ is an arbitrary orthonormal basis for $N$, the orthogonal complement of $T$. For the proof that the formula above is $(k$ times) the mean curvature vector see, for example, do Carmo's book [7].

2. Anisotropic motion by mean curvature

$$
v(S, T):=\sum_{i=1}^{n-k}\left(\operatorname{trace}\left(A(T) S_{n_{i}}\right)\right) n_{i} .
$$

If $k=1$ then the manifolds are curves and the velocity can be written as $v(\kappa, T)$, where $\kappa$ is the curvature vector and $T$ the tangent line.

3. $v(\kappa, T)=|\kappa|^{\alpha} \kappa$ for $\alpha>-1$.

4. $v(\kappa, T)=f(T) \kappa$, where $f$ is a positive function.

5. $v(\kappa, T)=|\kappa| \vec{f}(T)$, where $\vec{f}$ is a vector-valued function such that $\vec{f}(T)$ is orthogonal to $T$.

\subsection{Barriers, distance representation, and surrounding}

We first investigate the relationship between the codimension $n-k$ motion by velocity $v$ and the codimension 1 motion by velocity $V$. Unless otherwise specified, we always assume that the codimension $n-k$ velocity $v$ is smooth and that the codimension 1 velocity $V$ is continuous and nonincreasing in the $D n$ argument. We denote codimension $n-k$ velocities by small letters, and codimension 1 velocities by capital letters. Therefore we call them both just velocities, as it is clear what the codimension is.

Recall that to the velocity $V$ we always associate a function $F$ by

$$
F(X, p)=|p| V\left(\frac{1}{|p|}(I-\hat{p} \otimes \hat{p}) X(I-\hat{p} \otimes \hat{p}), \hat{p}\right) .
$$

Let us define the properties that we investigate. 
DEFINITION 4.2 Let $v$ and $V$ be velocities (as above). We say that $V$ is a barrier for $v$ if for every (generalized) evolution $\left\{\left(\Omega_{t}, \Upsilon_{t}\right)\right\}_{t \in[0, T]}$ by velocity $V$, and every classical evolution $\left\{\Gamma_{t}\right\}_{t \in[0, T]}$ by velocity $v$, the following implication holds: If $\Gamma_{0} \subset \Omega_{0} \cup \Upsilon_{0}$ then $\Gamma_{t} \subset \Omega_{t} \cup \Upsilon_{t}$ for all $t \in[0, T]$.

DEFINITION 4.3 Let $v$ and $V$ be velocities. We say that $V$ gives a distance representation to $v$ if for every compact connected $k$-dimensional manifold without boundary, $\Gamma$, and every $\psi \in$ $C^{2}\left(\Gamma \times[0, T], \mathbb{R}^{N}\right)$ that is an embedding for every fixed $t \in[0, T]$, the following two conditions are equivalent for $\Gamma_{t}:=\psi(\Gamma \times\{t\})$ :

(a) The manifolds $\Gamma_{t}$ move (in the classical sense) with velocity $v$ for $t \in(0, T)$; that is, for all $t \in(0, T)$ and all $x \in \Gamma$,

$$
\pi_{N_{y} \Gamma_{t}}\left(\frac{\partial \psi}{\partial t}(x, t)\right)=v\left(S \Gamma_{t}, T_{y} \Gamma_{t}\right)
$$

Here $y=\psi(x, t)$.

(b) $d_{t}+F\left(D^{2} d, D d\right) \geqslant 0$ on $A:=\{(z, t): t \in[0, T], d(z, t) \in(0, \sigma)\}$ for some $\sigma>0$. Here $d(x, t):=\operatorname{dist}\left(x, \Gamma_{t}\right)$.

DEFINITION 4.4 Let $V$ and $v$ be velocities. We say that $V$ surrounds $v$ if for every compact, connected $k$-dimensional manifold without boundary, $\Gamma$, and every $\psi \in C^{2}\left(\Gamma \times[0, T], \mathbb{R}^{N}\right)$ that is an embedding for every fixed $t \in[0, T]$, the following equivalence holds for $\Gamma_{t}:=\psi(\Gamma \times\{t\})$ : The manifolds $\Gamma_{t}$ evolve by velocity $v$ if and only if $\Gamma_{t}=\{x: u(x, t)=0\}$, where $u$ is the unique viscosity solution of the equation

$$
\begin{aligned}
& u_{t}+F\left(D^{2} u, D u\right)=0, \\
& u(x, 0)=u_{0}(x)
\end{aligned}
$$

Here $u_{0}$ is a nonnegative uniformly continuous function that is equal to zero on $\Gamma_{0}$ and positive on the complement of $\Gamma_{0}$.

We now list some general properties of these notions, and state the relations between them. But first we recall a useful lemma. For motion by mean curvature it follows from Lemma 3.11 and step 7 of the proof of Theorem 3.9 in [1]. For general motions the definition of viscosity solution by Ishii and Souganidis [14] needs to be used. The required modifications are straightforward.

Lemma 4.5 Let $V$ be a velocity, $\Gamma$ a compact $C^{2}$ manifold without boundary, and $\psi \in C^{2}(\Gamma \times$ $\left.[0, T], \mathbb{R}^{n}\right)$ an embedding for every fixed $t \in[0, T]$. Let $\Gamma_{t}:=\psi(\Gamma, t)$. Let $d(x, t):=\operatorname{dist}\left(x, \Gamma_{t}\right)$. If for some $\sigma>0$,

$$
d_{t}+F\left(D^{2} d, D d\right) \geqslant 0 \quad \text { on } A:=\{(x, t): d(x, t) \in(0, \sigma), t \in(0, T)\}
$$

then

$$
d_{t}+F\left(D^{2} d, D d\right) \geqslant 0 \quad \text { on } \mathbb{R}^{n} \times(0, T) \text { in the viscosity sense. }
$$

LEMMA 4.6 If the velocity $V$ gives a distance representation to $v$, then $V$ is a barrier for $v$.

Proof. Assume that $V$ gives a distance representation to $v$. Let $\left\{\Gamma_{t}\right\}_{t \in[0, T]}$ be a classical evolution by velocity $v$ and $\left\{\left(\Omega_{t}, \Upsilon_{t}\right)\right\}_{t \in[0, T]}$ a generalized evolution by velocity $V$, such that $\Gamma_{0} \subset \Omega_{0} \cup \Upsilon_{0}$.

Let $d(x, t):=\operatorname{dist}\left(x, \Gamma_{t}\right)$. Since $V$ gives a distance representation to $v$, for some $\sigma>0$ we have

$$
d_{t}(x, t)+F\left(D^{2} d(x, t), D d(x, t)\right) \geqslant 0 \quad \text { if } 0<d(x, t)<\sigma,
$$


for all $t \in[0, T]$. Lemma 4.5 implies that $d_{t}+F\left(D^{2} d, D d\right) \geqslant 0$ on $\mathbb{R}^{n} \times[0, T]$ in the viscosity sense.

By definition of generalized (codimension 1) evolutions, $\Omega_{t} \cup \Upsilon_{t}=\{x: u(x, t) \leqslant 0\}$, where $u$ is the viscosity solution of the equation

$$
\begin{aligned}
& u_{t}+F\left(D^{2} u, D u\right)=0, \\
& u(x, 0)=\operatorname{dist}_{ \pm}\left(x, \Upsilon_{0}\right),
\end{aligned}
$$

where dist $t_{ \pm}$is the signed distance to $\Upsilon_{0}$, negative in $\Omega_{0}$. Since $u \leqslant d$ at $t=0$, the comparison principle implies that $u \leqslant d$ for all $t \in[0, T]$. Therefore

$$
\Gamma_{t}=\{x: d(x, t)=0\} \subseteq\{x: u(x, t) \leqslant 0\}=\Omega_{t} \cup \Upsilon_{t} .
$$

The next lemma shows that surrounding implies giving a distance representation. The question when giving distance representation implies surrounding is open.

LEMMA 4.7 Let $v$ be such that for every compact, connected $k$-dimensional $C^{2}$ manifold without boundary embedded in $\mathbb{R}^{n}$ there exists a short time $C^{2}$ evolution by velocity $v$. If the velocity $V$ surrounds $v$, then it gives a distance representation to $v$.

REMARK. One of the reasons that we need to assume the short time existence is that surrounding and distance representation were both defined in a general way, without imposing any requirements on the existence of a classical evolution by velocity $v$. Although short time evolution exists for many velocities $v$, it clearly does not exist for all smooth velocities. For a velocity $v$ for which there are few manifolds that can evolve by it the equivalences in the definitions of surrounding and distance representation might not carry much information on relationship between $V$ and $v$. Our interest is clearly in the velocities by which many manifolds can evolve. Appropriate assumptions on short time existence are also needed in several other results, but the one that we need in this lemma is the strongest.

Proof. Assume that $V$ surrounds $v$. Let $\Gamma_{t}$ be as in the definition of distance representation.

$((\mathrm{a}) \Rightarrow(\mathrm{b})) \quad$ Assume that the manifolds $\Gamma_{t}$ move with velocity $v$. Since $V$ surrounds $v, \Gamma_{t}=$ $\{x: u(x, t)=0\}$, where $u$ is the viscosity solution of 2.1 with initial data $u(x, 0)=\operatorname{dist}\left(x, \Gamma_{0}\right)$. Let $d(x, t):=\operatorname{dist}\left(x, \Gamma_{t}\right)$.

Let $\varphi$ be an admissible (test) function such that $d-\varphi$ has a minimum at $\left(x_{0}, t_{0}\right)$. Let $x_{1}$ be a point on $\Gamma_{t_{0}}$ such that $d\left(x_{0}, t_{0}\right)=\left|x_{0}-x_{1}\right|$. Consider the function $\tilde{\varphi}(x, t):=\varphi\left(x+x_{0}-x_{1}, t\right)$. Then $d-\tilde{\varphi}$ has a minimum at $\left(x_{1}, t_{0}\right)$. We can assume that $\tilde{\varphi}\left(x_{1}, t_{0}\right)=0$ and that $\tilde{\varphi}<1$. Consider the function $H(d)$, where $H$ is defined by $H(x)=1$ if $x>0$ and $H(x)=0$ if $x \leqslant 0$. Note that $H(d)-\tilde{\varphi}$ has a minimum at $\left(x_{1}, t_{0}\right)$. Since $H(d)=H(u)=H(u)_{*}$, by property $(\mathrm{P} 1)$ the function $H(d)$ is a supersolution of $[2.1$. Therefore

$$
\begin{aligned}
\tilde{\varphi}_{t}\left(x_{1}, t_{0}\right)+F\left(D^{2} \tilde{\varphi}\left(x_{1}, t_{0}\right), D \tilde{\varphi}\left(x_{1}, t_{0}\right)\right) \geqslant 0 & \text { if } D \tilde{\varphi}\left(x_{1}, t_{0}\right) \neq 0 \\
\tilde{\varphi}_{t}\left(x_{1}, t_{0}\right) \geqslant 0 & \text { if } D \tilde{\varphi}\left(x_{1}, t_{0}\right)=0
\end{aligned}
$$

Consequently, $\varphi_{t}\left(x_{0}, t_{0}\right)+F\left(D^{2} \varphi\left(x_{0}, t_{0}\right), D \varphi\left(x_{0}, t_{0}\right)\right) \geqslant 0$ if $D \varphi\left(x_{0}, t_{0}\right) \neq 0$, and $\varphi_{t}\left(x_{0}, t_{0}\right) \geqslant 0$ if $D \varphi\left(x_{0}, t_{0}\right)=0$. Therefore $d$ is a viscosity supersolution of 2.1). By Lemma 3.2, there exists $\sigma>0$ such that $d$ is twice differentiable on the set where $0<d<\sigma$. Therefore (b) holds, since viscosity supersolutions that are differentiable, are classical supersolutions. 
$((\mathrm{b}) \Rightarrow(\mathrm{a})) \quad$ Assume that

$$
d_{t}+F\left(D^{2} d, D d\right) \geqslant 0 \quad \text { when } 0<d<\sigma
$$

for some $\sigma>0$. By Lemma 4.5 we know that $d$ is a viscosity supersolution on the whole space.

Let $\left\{\tilde{\Gamma}_{t}\right\}_{t \in\left[0, t_{\max }\right)}$ be the $C^{2}$ evolution of $\Gamma_{0}$ by velocity $v$, where $\left[0, t_{\max }\right)$ is the maximal interval on which the $C^{2}$ evolution exists (note that it has to be open on the right).

For $u$ as above, by definition of surrounding $\{x: u(x, t)=0\}=\tilde{\Gamma}_{t}$ for $t \in\left[0, t_{\max }\right)$. By the comparison principle $d(x, t) \geqslant u(x, t)$ for all $x \in \mathbb{R}^{n}$ and $t \in[0, T]$. Therefore $\Gamma_{t} \subseteq \tilde{\Gamma}_{t}$ for $t \in[0, T] \cap\left[0, t_{\max }\right)$. Since $\Gamma_{t}$ is both open and closed in $\tilde{\Gamma}_{t}$, and $\tilde{\Gamma}_{t}$ is connected, we conclude that $\Gamma_{t}=\tilde{\Gamma}_{t}$ for $t \in[0, T] \cap\left[0, t_{\max }\right)$. To finish the proof we need that $t_{\max }>T$. But if $t_{\max } \leqslant T$ then by defining $\tilde{\Gamma}_{t_{\max }}=\Gamma_{t_{\max }}$ we can extend the $C^{2}$ evolution beyond $\left[0, t_{\max }\right.$ ), which is impossible.

LEMMA 4.8 Velocity $V$ is a barrier for velocity $v$ if and only if for every classical evolution $\left\{\Gamma_{t}\right\}_{t \in[0, T]}$ by velocity $v$, there exists $\sigma>0$ such that

$$
d_{t}(x, t)+F\left(D^{2} d(x, t), D d(x, t)\right) \geqslant 0 \quad \text { when } d(x, t) \in(0, \sigma),
$$

where $d(x, t):=\operatorname{dist}\left(x, \Gamma_{t}\right)$.

Proof. Assume that $V$ is a barrier for $v$. Let $\left\{\Gamma_{t}\right\}_{t \in[0, T]}$ be a classical evolution by velocity $v$. Assume that the conclusion of the lemma does not hold. Then for $\sigma$ as in Lemma 3.2, there exist $t_{0} \in(0, T)$ and $x_{0}$ such that $0<d\left(x_{0}, t_{0}\right)<\sigma$ and

$$
-2 \varepsilon:=d_{t}\left(x_{0}, t_{0}\right)+F\left(D^{2} d\left(x_{0}, t_{0}\right), \operatorname{Dd}\left(x_{0}, t_{0}\right)\right)<0 .
$$

Let $y \in \Gamma_{t_{0}}$ be such that $\left|x_{0}-y\right|=d\left(x_{0}, \Gamma_{t_{0}}\right)$ and let $p:=\widehat{x_{0}-y}$.

For $s \in(0,1]$ let $\Omega_{t_{0}}^{s}:=\left\{x: d\left(x, \Gamma_{t_{0}}\right)<s d\left(x_{0}, \Gamma_{t_{0}}\right)\right\}$ and $\Upsilon_{t_{0}}^{s}=\partial \Omega_{t_{0}}^{s}$. Denote by $\left\{\left(\Omega_{t}^{s}, \Upsilon_{t}^{s}\right)\right\}_{t \in\left[t_{0}, T\right]}$ the generalized evolution of $\left(\Omega_{t_{0}}^{s}, \Upsilon_{t_{0}}^{s}\right)$ by velocity $V$.

Let $\tau_{x}$ be the translation (in $\mathbb{R}^{n}$ ) by vector $x$. Let $\Gamma_{t}^{s}:=\tau_{s\left(x_{0}-y\right)}\left(\Gamma_{t}\right)$. Note that $\left\{\Gamma_{t}^{s}\right\}_{t \in[0, T]}$ is an evolution by velocity $v$ for all $s \in(0,1]$. Also note that $\Gamma_{t_{0}}^{s} \subset \Omega_{t_{0}}^{s} \cup \Upsilon_{t_{0}}^{s}$. To obtain a contradiction it is enough to show that for some $s, t \in\left(t_{0}, T\right], \Gamma_{t}^{s} \not \subset \Omega_{t}^{s} \cup \Upsilon_{t}^{s}$.

Consider a $C^{2}$ function $W$ on $\Upsilon_{t_{0}}$ such that for all $x \in \Upsilon_{t_{0}}, V\left(D^{2} d\left(x, t_{0}\right), D d\left(x, t_{0}\right)\right)<W(x)<$ $V\left(D^{2} d\left(x, t_{0}\right), D d\left(x, t_{0}\right)\right)+\varepsilon$. Let $\Psi: \Upsilon_{t_{0}} \times\left[t_{0}, t_{0}+\delta\right] \rightarrow \mathbb{R}^{n}$ be given by $\Psi(x, t):=x+W(x) t$. Since $\Upsilon_{t_{0}}$ is compact, there exists $\delta>0$ such that $\Psi(\cdot, t)$ is an embedding for all $t \in\left[t_{0}, t_{0}+\delta\right]$, and the manifolds $\tilde{\Upsilon}_{t}:=\Psi\left(\Upsilon_{t_{0}}, t\right)$ (along with the open sets $\tilde{\Omega}_{t}$ they enclose) form a superflow by velocity $V$. Therefore by comparison, for all $s \in(0,1)$ and $t \in\left[t_{0}, t_{0}+\delta\right], \Omega_{t}^{s} \cup \Upsilon_{t}^{s} \subseteq \tilde{\Omega}_{t} \cup \tilde{\Upsilon}_{t}$. From the definition of $C$ and 3.5 it now follows that

$$
v\left(S \Gamma_{t_{0}}^{1}, T_{x_{0}} \Gamma_{t_{0}}^{1}\right) \cdot p=v\left(S \Gamma_{t_{0}}, T_{y} \Gamma_{t_{0}}\right) \cdot p>V\left(D^{2} d\left(x_{0}\right), p\right)+\varepsilon .
$$

In other words, the velocity of $\Gamma_{t}^{1}$ at $\left(x_{0}, t_{0}\right)$ in the direction of vector $p$ (which is the normal vector to $\tilde{\Upsilon}_{t_{0}}$ at $\left.x_{0}\right)$ is greater than the normal velocity of $\tilde{\Upsilon}_{t}$ at $\left(x_{0}, t_{0}\right)$. Therefore there exists $t \in\left(t_{0}, T\right)$ such that $\Gamma_{t}^{1} \backslash\left(\tilde{\Omega}_{t} \cup \tilde{\Upsilon}_{t}\right) \neq \emptyset$. Hence for $s$ close enough to $1, \Gamma_{t}^{s} \backslash\left(\tilde{\Omega}_{t} \cup \tilde{\Upsilon}_{t}\right) \neq \emptyset$. But since $V$ is a barrier for $v, \Gamma_{t}^{s} \subset \Omega_{t}^{s} \cup \Upsilon_{t}^{s} \subseteq \tilde{\Omega}_{t} \cup \tilde{\Upsilon}_{t}$. Contradiction.

To show the other implication, let $\left\{\Gamma_{t}\right\}_{t \in[0, T]}$ be a classical flow by velocity $v$ for which for some $\sigma>0$,

$$
d_{t}(x, t)+F\left(D^{2} d(x, t), D d(x, t)\right) \geqslant 0 \quad \text { when } d(x, t) \in(0, \sigma) .
$$

From this point on the argument is identical to the one in Lemma 4.6 
The following observation follows from the definitions, the properties of viscosity solutions and the previous lemma.

LEMMA 4.9 If $V_{1}$ is a barrier for $v$ and $V_{2}$ is a barrier for (resp. gives a distance representation to, surrounds) $v$ then $V:=\min \left\{V_{1}, V_{2}\right\}$ is a barrier for (resp. gives a distance representation to, surrounds) $v$.

We now turn our attention to discussing necessary and sufficient conditions for velocity $V$ to give a distance representation to $v$.

Conditions $(\diamond)$ and $(\star)$. Let $T$ be a $k$-dimensional plane in $\mathbb{R}^{n}, N$ its orthogonal complement, and $p$ a unit vector in $N$. Let $\left[t_{1}, \ldots, t_{k}\right]$ be an orthonormal basis of $T$ and $\left[t_{k+1}, \ldots, t_{n}\right]$ an orthonormal basis of $N$ such that $t_{n}=p$. Let $S: N \times T \rightarrow T$ be a bilinear mapping. By $S_{p}$ we denote the matrix of $S(p, \cdot)$ written in the basis $\left[t_{1}, \ldots, t_{k}\right]$. Let $R$ be the matrix $\left[t_{1}, \ldots, t_{n}\right]$.

We say that condition ( $\diamond)$ is satisfied if for all $T, S, R, p$ as above,

$$
\lim _{s \rightarrow 0^{+}} V\left(R \operatorname{diag}\left(-S_{p}\left(I_{k}-s S_{p}\right)^{-1}, \frac{1}{s} I_{n-k-1}, 0\right) R^{-1}, p\right) \geqslant v(S, T) \cdot p .
$$

We say that condition ( $\star$ ) holds if for all $T, S, R, p$ as above,

$$
\lim _{s \rightarrow 0^{+}} V\left(R \operatorname{diag}\left(-S_{p}\left(I_{k}-s S_{p}\right)^{-1}, \frac{1}{s} I_{n-k-1}, 0\right) R^{-1}, p\right)=v(S, T) \cdot p .
$$

Here $\operatorname{diag}\left(A_{1}, \ldots, A_{m}\right)$ is a quasidiagonal matrix with matrices $A_{1}, \ldots, A_{m}$ along the diagonal.

THEOREM 4.10 If for velocities $V$ and $v$ condition $(\diamond)$ holds then $V$ is a barrier for $v$. If condition $(\star)$ holds then $V$ gives a distance representation to $v$.

Proof. Assume that for $v$ and $V$ condition $(\diamond)$ holds. Let $\left\{\Gamma_{t}\right\}_{t \in[0, T]}$ be a classical evolution by velocity $v$. Let $\sigma, A, X(t)$ and $\Pi$ be as in Lemma 3.2 Let $z \in A, y:=\Pi(z, t), s=|z-y|$, and $p:=\widetilde{z-y}$. From 3.5 it follows that

$$
\frac{\partial d}{\partial t}(z, t)=-p \cdot \frac{\partial \psi}{\partial t}(X(t), t)=-p \cdot v\left(S \Gamma_{t}, T_{y} \Gamma_{t}\right)
$$

since $\Gamma_{t}$ evolve by velocity $v$. Therefore, using the fact that $F$ is degenerate elliptic and condition $(\diamond)$, we obtain

$$
\begin{aligned}
d_{t}(z, t)+F( & \left.D^{2} d(z, t), D d(z, t)\right) \\
& =d_{t}(z, t)+F\left(R \operatorname{diag}\left(-S_{p}\left(I_{k}-s S_{p}\right)^{-1}, \frac{1}{s} I_{n-k-1}, 0\right) R^{-1}, p\right) \\
& \geqslant d_{t}(z, t)+\lim _{s \rightarrow 0^{+}} F\left(R \operatorname{diag}\left(-S_{p}\left(I_{k}-s S_{p}\right)^{-1}, \frac{1}{s} I_{n-k-1}, 0\right) R^{-1}, p\right) \\
& =-p \cdot v\left(S \Gamma_{t}, T_{y} \Gamma_{t}\right)+p \cdot v\left(S \Gamma_{t}, T_{y} \Gamma_{t}\right)=0 .
\end{aligned}
$$

Lemma 4.8 then implies that $V$ is a barrier for $v$.

Let us now assume that condition $(\star)$ holds.

((a) $\Rightarrow$ (b)) Assume that the condition (a) of the definition of distance representation holds. Since condition $(\star)$ implies $(\diamond)$ the calculation above shows that (b) holds. 
((b) $\Rightarrow$ (a)) Assume that (b) holds. Let $x \in \Gamma$ and $t \in(0, T)$ be arbitrary. Let $y:=\psi(x, t)$. To show (a) it is enough to show that for every $p \in N_{y} \Gamma_{t}$,

$$
\frac{\partial \psi}{\partial t}(x, t) \cdot p=v\left(S \Gamma_{t}, T_{y} \Gamma_{t}\right) \cdot p .
$$

So let $p \in N_{y} \Gamma_{t}$ be arbitrary. From 3.5 we have $d_{t}(z, t)=-p \cdot \frac{\partial \psi}{\partial t}(x, t)$. For all $s \in(0, \sigma)$, by the assumption that (b) holds, we have

$$
-p \cdot \frac{\partial \psi}{\partial t}(x, t)+F\left(D^{2} d(y+s p), p\right) \geqslant 0 .
$$

We have computed $D^{2} d$ in 3.4. By using that and taking the limit as $s \rightarrow 0$ we obtain

$$
-p \cdot \frac{\partial \psi}{\partial t}(x, t)+\lim _{s \rightarrow 0^{+}} F\left(R \operatorname{diag}\left(-S_{p}\left(I_{k}-s S_{p}\right)^{-1}, \frac{1}{s} I_{n-k-1}, 0\right) R^{-1}, p\right) \geqslant 0,
$$

which by assumption $(\star)$ implies

$$
p \cdot\left(\frac{\partial \psi}{\partial t}(x, t)+v\left(S \Gamma_{t}, T_{y} \Gamma_{t}\right)\right) \geqslant 0 .
$$

Since $p$ was an arbitrary normal vector, the previous claim holds for vector $-p$ as well:

$$
-p \cdot\left(\frac{\partial \psi}{\partial t}(x, t)+v\left(S \Gamma_{t}, T_{y} \Gamma_{t}\right)\right) \geqslant 0
$$

Therefore (4.4) holds.

EXAMPLE 1. This theorem can be applied to the motion by mean curvature vector:

$$
v(S, T)=\sum_{i=1}^{n-k}\left(\operatorname{trace} S_{n_{i}}\right) n_{i} .
$$

Here $\left[n_{1}, \ldots, n_{n-k}\right]$ is an arbitrary orthonormal basis of $N$. Let $V$ be minus the sum of the $k$ smallest principal curvatures. To be more precise let us define $F: \operatorname{Sym}(n) \times \mathbb{R}^{n} \backslash\{0\} \rightarrow \mathbb{R}$. For $X \in \operatorname{Sym}(n)$ and $p \in \mathbb{R}^{n} \backslash\{0\}$ let $\lambda_{1} \leqslant \cdots \leqslant \lambda_{n-1}$ be the eigenvalues of $(I-\hat{p} \otimes \hat{p}) X(I-\hat{p} \otimes \hat{p})$ that correspond to eigenvectors orthogonal to $p$. Let $F(X, p):=-\left(\lambda_{1}+\cdots+\lambda_{k}\right)$. Note that condition $(\star)$ is satisfied:

$$
\begin{aligned}
\lim _{s \rightarrow 0^{+}} V\left(R \operatorname { d i a g } \left(-S_{p}\left(I_{k}-s S_{p}\right)^{-1}\right.\right. & \left.\left., \frac{1}{s} I_{n-k-1}, 0\right) R^{-1}, p\right) \\
& =\lim _{s \rightarrow 0^{+}} \operatorname{trace}\left(S_{p}\left(I_{k}-s S_{p}\right)^{-1}\right)=\operatorname{trace} S_{p}=v(S, T) \cdot p .
\end{aligned}
$$

Therefore the conclusion of the lemma applies. This distance representation of mean curvature motion was first obtained by Ambrosio and Soner [1, Theorem 3.8]. Note that the $V$ we used is not the only velocity that satisfies condition $(\star)$ for the mean curvature velocity. For example

$$
\tilde{F}(X, p)=-\left(\lambda_{1}+\cdots+\lambda_{k}\right)+ \begin{cases}1 / \lambda_{n-1} & \text { if } \lambda_{n-1}>1, \\ 1 & \text { otherwise }\end{cases}
$$


gives another velocity that satisfies $(\star)$. However, note that $\tilde{F} \geqslant F$. We show later (for the evolution of curves) that $V$ is the smallest velocity that satisfies $(\star)$, and furthermore that it is the smallest velocity that gives a distance representation to $v$.

EXAMPLE 2. Further examples of velocities that satisfy ( $\star$ ) can be obtained from the previous example by making a linear transformation of the space and expressing the normal component of the velocity in the new variables. Let us just illustrate that in the case of the motion of curves in $\mathbb{R}^{n}$. Let $P$ be a nondegenerate $n \times n$ matrix. Let

$$
v_{P}(\kappa, t):=\frac{1}{\left|P^{-1} t\right|^{2}} \kappa
$$

where $\kappa$ is the curvature vector and $t$ a unit tangent vector, and let $V_{P}(D n, n)$ be the greatest eigenvalue of the matrix

$$
A:=-\left(I-\frac{P^{T} n \otimes P^{T} n}{\left|P^{T} n\right|^{2}}\right) P^{T} D n P\left(I-\frac{P^{T} n \otimes P^{T} n}{\left|P^{T} n\right|^{2}}\right)
$$

that corresponds to an eigenvector orthogonal to $P^{T} n$. It is not difficult (using the fact that $\left.V_{P}(D n, n)=\max \left\{A x \cdot x:|x|=1, x \cdot P^{T} n=0\right\}\right)$ to check that $V_{P}$ and $v_{P}$ satisfy condition $(\star)$.

We have shown that condition ( $\star$ ) is sufficient for conditions (a) and (b) to be equivalent. As can be seen in Section 5 it is not a necessary condition. In this generality we cannot say what condition is both sufficient and necessary. Nevertheless, some improvements to condition ( $\star$ ) can be made. For example, it is enough to require that

$$
\lim _{s \rightarrow 0^{+}} V\left(R \operatorname{diag}\left(-S_{p}\left(I_{k}-s S_{p}\right)^{-1}, \frac{1}{s} I_{n-k-1}, 0\right) R^{-1}, p\right)=v(S, T) \cdot p
$$

only for all $p \in\left\{p_{1}, \ldots, p_{n-k},-p_{1}, \ldots,-p_{n-k}\right\}$ where $\left[p_{1}, \ldots, p_{n-k}\right]$ is an arbitrary orthonormal basis of $N$. The proof that this is a sufficient condition as well is essentially the same as the one given for $(\star)$.

LEMMA 4.11 Let $v$ be a velocity by which every smooth, connected, and compact $k$-dimensional manifold without boundary embedded in $\mathbb{R}^{n}$ has a short time classical evolution. If $V$ is a barrier for $v$ then condition $(\diamond)$ holds.

Proof. Assume otherwise. Then there exist $T, S$ and $p$ for which condition $(\diamond)$ does not hold. Hence there exists $s_{0}>0$ such that for all $s \in\left(0, s_{0}\right)$,

$$
V\left(R \operatorname{diag}\left(-S_{p}\left(I_{k}-s S_{p}\right)^{-1}, \frac{1}{s} I_{n-k-1}, 0\right) R^{-1}, p\right)<v(S, T) \cdot p .
$$

Let $\Gamma_{0}$ be a smooth, connected, and compact $k$-dimensional manifold embedded in $\mathbb{R}^{n}$ such that $0 \in \Gamma_{0}$ and $T$ is the tangent plane to $\Gamma_{0}$ at 0 , and $S$ the shape operator at 0 . Let $\Gamma_{t}$ be the classical short time evolution of $\Gamma_{0}$ by velocity $v$. Let $s \in\left(0, s_{0}\right)$. By using (3.5) and computing as in 4.1) we get

$$
d_{t}(s p, 0)+V\left(D^{2} d(s p, 0), p\right)<-p \cdot v(S, T)+p \cdot v(S, T)=0 .
$$

But then, by Lemma $4.8, V$ is not a barrier for $v$. Contradiction. 
COROLlARY 4.12 Let $v$ be a velocity that satisfies the assumptions of the previous lemma. For velocity $V$ to give a distance representation to $v$ it is necessary that condition $(\diamond)$ holds.

At the end of this section we present a sufficient condition for a velocity $V$ to surround a velocity $v$.

Condition $(\mathbf{\Delta})$. We say that velocity $V$ satisfies condition $(\boldsymbol{\Delta})$ if for every $M>0$ there exists $K>0$ such that for all $k \times k$ matrices $S$ such that $\|S\|<M$ and all orthogonal matrices $R=$ $\left[l_{1}, \ldots, l_{n-1}, p\right]$ the following holds for $0<s^{\prime}<s<1 / K$ :

$$
\begin{aligned}
V\left(R \operatorname{diag}\left(-S\left(I_{k}-s S\right)^{-1}, \frac{1}{s} I_{n-k-1}, 0\right) R^{-1}, p\right) \\
\quad-V\left(R \operatorname{diag}\left(-S\left(I_{k}-s^{\prime} S\right)^{-1}, \frac{1}{s^{\prime}} I_{n-k-1}, 0\right) R^{-1}, p\right) \leqslant O\left(s^{\prime}\right)+K s .
\end{aligned}
$$

LEMMA 4.13 If velocities $V$ and $v$ satisfy condition ( $\star$ ) and $V$ satisfies $(\boldsymbol{\Delta})$ then $V$ surrounds $v$.

The proof of this lemma is a straightforward generalization of the proof of Corollary 3.9 in [1]. Let us also remark that velocities $V_{P}$ and $v_{P}$ given in Example 2 satisfy the conditions of this lemma, and hence $V_{P}$ surrounds $v_{P}$.

\section{Motion of curves along the normal vector}

We now turn our attention to the motion of curves in $\mathbb{R}^{n}$, the case that we can more fully investigate. In this case the tangent space is one-dimensional and therefore the shape operator has a very simple form.

In general, for a one-dimensional subspace, $T$, of $\mathbb{R}^{n}$, its orthogonal complement $N$, and a bilinear mapping $S: N \times T \rightarrow T$ there exists a vector $\kappa \in N$ such that $S(a, b)=(\kappa \cdot a) b$ for all $a \in N, b \in T$. For a point on a curve, and the shape operator $S, \kappa$ is the curvature vector. Therefore the velocities that we are considering can be written as a function of the tangent space $T$ and the curvature vector $\kappa$.

We devote most of this section to studying velocities that have the direction of the normal vector, but let us begin with a proposition that gives a necessary condition for a general velocity to have a distance representation.

Proposition 5.1 Let $v(\kappa, T)$ be a velocity by which every circle can evolve for a short time. If it has a distance representation then the component of $v(\kappa, T)$ orthogonal to $\kappa$ is bounded, and furthermore, there exists a constant $C$ such that $|v(\kappa, T)| \leqslant C|\kappa|$ when $|\kappa| \geqslant 1$.

Proof. Let $V$ be a velocity that gives a distance representation to $v$. Note that condition $(\diamond)$ then holds (see the proof of Lemma 4.11). From $(\diamond)$ it follows that for $p$ a unit vector orthogonal to $\kappa$,

$$
V(0, p) \geqslant v(\kappa, T) \cdot p
$$

For a vector $x$ denote by $x^{\perp}$ the set of unit vectors orthogonal to $x$. Then

$$
M:=\sup _{p \in S^{n-1}} V(0, p) \geqslant \sup _{\kappa, T} \sup _{p \in \kappa^{\perp}} v(\kappa, T) \cdot p .
$$

To prove the second claim we only need to bound the component of $v(\kappa, T)$ in the direction of $\kappa$. Given $\kappa$ such that $|\kappa| \geqslant 1$ and $T$, let $n:=\hat{\kappa}$. Choose $p$ such that $n \cdot p=1 /|\kappa|$, let 
$R=\left[l_{1}, \ldots, l_{n-1}, p\right]$ be an orthogonal matrix such that $T=\operatorname{span}\left\{l_{1}\right\}$, and let $s=1 / 2$. Then $(\diamond)$ yields

$$
V\left(R \operatorname{diag}(-2,2, \ldots, 2,0) R^{-1}, p\right) \geqslant v(\kappa, T) \cdot p \geqslant \frac{v(\kappa, T) \cdot n}{|\kappa|}-M,
$$

while choosing $p$ so that $-n \cdot p=1 /|\kappa|$ yields

$$
-V\left(R \operatorname{diag}(2 / 3,2, \ldots, 2,0) R^{-1}, p\right) \leqslant-v(\kappa, T) \cdot p \leqslant \frac{v(\kappa, T) \cdot n}{|\kappa|}+M .
$$

Setting $C:=\max \left\{M+\left|V\left(R \operatorname{diag}(-2,2, \ldots, 2,0) R^{-1}, p\right)\right|+\left|V\left(R \operatorname{diag}\left(\frac{2}{3}, 2, \ldots, 2,0\right) R^{-1}, p\right)\right|:\right.$ $\left.p \in S^{n-1}, R^{-1}=R^{T}, R e_{n}=p\right\}$ completes the proof.

The velocities that have the direction of the unit normal vector can be described by a scalar function that we now introduce. For $k \geqslant 0$ and mutually orthogonal vectors $n$ and $t$ let

$$
v(k, n, t):=v(k n, \operatorname{span}\{t\}) \cdot n .
$$

Note that $v(k, n, t)$ is even in the $t$ variable, and that since $v(\kappa, T)$ is smooth, so is $v(k, n, t)$. Also note that continuity of $v(\kappa, T)$ at $\kappa=0$ implies that $v(0, \cdot)=0$ and hence $v(k, \cdot, \cdot)=0$. Let us now loosen the requirements on $v(k, n, t)$ a bit. From now on, we only require that $v(k, n, t)$ is continuous on its domain and smooth for $k>0$. Also, from now on, when we say velocity $v$ we have in mind velocity $v(k, n, t)$ along the normal vector.

For velocities $V$ that we are considering, we assume that

$$
V\left(R \operatorname{diag}\left(0, \frac{1}{s} I_{n-2}, 0\right) R^{-1}, p\right)=0
$$

for all $s>0$, all unit vectors $p$, and all orthogonal matrices $R$ for which $R p=e_{n}$. Let us show that this condition is not restrictive, in the following sense: For a given velocity $v$ for which every circle has a short time evolution, in Lemma 5.2 we build a velocity $V^{\prime}$ that is a barrier for $v$ and satisfies condition 5.1. For every velocity $V$ that gives a distance representation to $v$, Lemma 4.9 tells us that $\min \left\{V, V^{\prime}\right\}$ also gives a distance representation to $v$. So, if $v$ has a distance representation then there exists a velocity that satisfies $(5.1)$ and gives $v$ a distance representation.

LEMMA 5.2 Let $v(k, n, t)$ be a velocity that has a distance representation and by which every circle has a short time classical evolution. Then $v$ has a barrier $V^{\prime}$ such that

$$
V^{\prime}\left(R \operatorname{diag}\left(0, \frac{1}{s} I_{n-2}, 0\right) R^{-1}, p\right)=0
$$

for all $s>0$, all unit vectors $p$, and all orthogonal matrices $R$ such that $R e_{n}=p$.

The proof of this lemma relies on Theorem 5.4 and constructions of Definition 5.9, so the reader may wish to postpone reading the proof.

Proof. Since $v$ has a distance representation, by Theorem 5.4 it is nonnegative and nondecreasing in $k$. By Proposition 5.1 there exists $C>0$ such that if $k \geqslant 1$ then $C k \geqslant v(k, n, t)$. Let $v_{1}(k):=$ $\max \{v(k, n, t):|n|=|t|=1, n \cdot t=0\}$. Let $v_{2}:=\bar{U} v_{1}$, where $\bar{U}$ is defined in Definition 5.9 . Note that $v_{2}$ is continuous. Let

$$
V^{\prime}\left(R \operatorname{diag}\left(k_{1}, \ldots, k_{n-1}, 0\right) R^{-1}, p\right):= \begin{cases}0 & \text { if } k_{i} \geqslant 0 \text { for all } i \\ v_{2}\left(-\min \left\{k_{1}, \ldots, k_{n-1}\right\}\right) & \text { otherwise. }\end{cases}
$$


Let $k \geqslant 0$, let $n, t$, and $p$ be unit vectors such that $n \cdot t=0$, and let $R$ be an orthogonal matrix such that $R e_{n}=p$. Let us check if condition $(\diamond)$ holds. If $n \cdot p>0$ then for all $s>0$ small enough

$$
\begin{aligned}
V^{\prime}\left(R \operatorname{diag}\left(\frac{-k n \cdot p}{1-s k n \cdot p}, \frac{1}{s} I_{n-1}, 0\right) R^{-1}, p\right) & =v_{2}\left(\frac{k n \cdot p}{1-s k n \cdot p}\right) \\
& \geqslant \frac{n \cdot p}{1-s k n \cdot p} v_{2}(k) \geqslant v(k, n, t) n \cdot p .
\end{aligned}
$$

If $n \cdot p<0$ and $s>0$ is small enough then

$$
V^{\prime}\left(R \operatorname{diag}\left(\frac{-k n \cdot p}{1-s k n \cdot p}, \frac{1}{s} I_{n-1}, 0\right) R^{-1}, p\right)=0 \geqslant v(k, n, t) n \cdot p .
$$

By Theorem 4.10, we now conclude that $V^{\prime}$ is a barrier for $v$.

\subsection{Necessary and sufficient conditions for distance representation}

Let us introduce the following notation: For a vector $x$ let $x^{+}:=\left\{y \in S^{n-1}: x \cdot y>0\right\}$ and $x^{-}:=(-x)^{+}$. Recall that $x^{\perp}$ is the set of unit vectors orthogonal to $x$. For given velocity $V, k \geqslant 0$ and mutually orthogonal unit vectors $n$ and $t$ let

$$
\begin{aligned}
\bar{v}[V](k, n, t) & :=\inf _{p \in t^{\perp} \cap n^{+}} \lim _{s \rightarrow 0^{+}} \frac{V\left(R \operatorname{diag}\left(-\frac{k n \cdot p}{1-s k n \cdot p}, \frac{1}{s} I_{n-2}, 0\right) R^{-1}, p\right)}{n \cdot p}, \\
\underline{v}[V](k, n, t) & :=\sup _{p \in t^{\perp} \cap n^{-}} \lim _{s \rightarrow 0^{+}} \frac{V\left(R \operatorname{diag}\left(-\frac{k n \cdot p}{1-s k n \cdot p}, \frac{1}{s} I_{n-2}, 0\right) R^{-1}, p\right)}{n \cdot p},
\end{aligned}
$$

where $R$ is an orthogonal matrix such that $R e_{1}=t$ and $R e_{n}=p$.

Condition ( $\star \star)$ ). We say that velocities $v$ and $V$ satisfy condition $(\star \star)$ ) if for all $k>0$, and all mutually orthogonal vectors $n$ and $t, v(k, n, t)$ is nonnegative and

$$
\bar{v}[V](k, n, t)=\underline{v}[V](k, n, t)=v(k, n, t) .
$$

REMARK. We are about to show that this condition is sufficient, and close to being necessary, for a velocity $V$ to give a distance representation to a velocity $v$. Nonetheless, it can still be weakened a bit (and still remain sufficient). Requiring that $v(k, n, t)$ is nonnegative and that $\bar{v}[V](k, n, t) \geqslant$ $v(k, n, t)$ and $\underline{v}[V](k, n, t) \leqslant v(k, n, t)$ is necessary. But instead of equality it is enough to require that for every $k_{0}>0$, and mutually orthogonal vectors $n_{0}$ and $t_{0}$, there exists a neighborhood $U$ of $\left(k_{0}, n_{0}, t_{0}\right)$ such that either for all $(k, n, t) \in U$ such that $t \neq t_{0}$, or for all $(k, n, t) \in U$ such that $n \neq n_{0}, \bar{v}[V](k, n, t) \leqslant v(k, n, t)$ and $\underline{v}[V](k, n, t) \geqslant v(k, n, t)$. Or better yet, it is enough to require the following: For every smooth curve $\rho:(-a, a) \rightarrow S^{n-1}$ such that $\rho^{\prime}(0) \neq 0$, zero is an accumulation point of the set $\left\{s:\left(\left|\rho^{\prime}(s)\right|, \widehat{\rho^{\prime}(s)}, \rho(s)\right) \in W\right\}$. Here $W$ is the set of $(k, n, t)$ for which $\bar{v}[V](k, n, t) \leqslant v(k, n, t)$ and $\underline{v}[V](k, n, t) \geqslant v(k, n, t)$. Theorem 5.3 would remain true if the weakened condition was used. Heuristics is that, knowing the velocity on a dense set of points on a $C^{2}$ evolving curve determines the velocity at every point. These small improvements of condition $(\star \star)$ are somewhat cumbersome, so we chose to use $(\star \star)$ instead, and occasionally comment on possible improvements. 
THEOREM 5.3 Let $v$ be a velocity and $V$ a velocity that satisfies condition (5.1). If condition $(\star \star)$ ) holds then $V$ gives a distance representation to $v$.

Proof. $\quad((\mathrm{a}) \Rightarrow(\mathrm{b})) \quad$ The proof is the same as in Theorem 4.10 since condition $(\star \star)$ implies $(\diamond)$.

$((b) \Rightarrow(a)) \quad$ We begin as in the proof of Theorem 4.10. Assume that claim (b) holds. Let $x \in \Gamma$ and $t \in(0, T)$ be arbitrary. Let $y:=\psi(x, t)$ and let $p \in N_{y} \Gamma_{t}$ be arbitrary. From (3.5) we have $d_{t}(z, t)=-p \cdot \frac{\partial \psi}{\partial t}(x, t)$. For all $s \in(0, \sigma)$, by the assumption that (b) holds, we have

$$
-p \cdot \frac{\partial \psi}{\partial t}(x, t)+F\left(D^{2} d(y+s p), p\right) \geqslant 0 .
$$

Consider first the vectors $p$ orthogonal to $n$. In 3.4 we computed that $D^{2} d(y+s p)=$ $R \operatorname{diag}\left(0, \frac{1}{s} I_{n-2}, 0\right) R^{-1}$ for an appropriate orthogonal matrix $R$. From condition $\sqrt{5.1}$ it follows that $F\left(D^{2} d(y+s p), p\right)=0$ and so $-p \cdot \frac{\partial \psi}{\partial t}(x, t) \geqslant 0$. Since the same holds if $p$ is replaced by $-p$ we conclude that

$$
p \cdot \frac{\partial \psi}{\partial t}(x, t)=0 \quad \text { for all } p \in N_{y} \Gamma_{t} \text { such that } n \cdot p=0 .
$$

If $k=0$ this completes the proof, since $v(0, T)=0$. So we can assume that $k \neq 0$. We know by now that $\pi_{N} \frac{\partial \psi}{\partial t}(x, t)=\tilde{v} n$ for some real number $\tilde{v}$. From 5.4 it now follows that for all $p \in N_{y} \Gamma_{t}$,

$$
-\tilde{v} p \cdot n+F\left(D^{2} d(y+s p), p\right) \geqslant 0 .
$$

Using (3.4), dividing by $n \cdot p$ and taking the infimum and the limit yields

$$
\inf _{p \in t^{\perp} \cap n^{+}} \lim _{s \rightarrow 0^{+}} \frac{V\left(R \operatorname{diag}\left(-\frac{k n \cdot p}{1-s k n \cdot p}, \frac{1}{s} I_{n-2}, 0\right) R^{-1}, p\right)}{n \cdot p} \geqslant \tilde{v} .
$$

Assumption $(\star \star)$ implies $v(k, n, t) \geqslant \tilde{v}$. On the other hand, dividing by $-n \cdot p$ and repeating the procedure yields $-v(k, n, t) \geqslant-\tilde{v}$. Therefore $\pi_{N} \frac{\partial \psi}{\partial t}(x, t)=\tilde{v} n=v(k, n, t) n$.

We are now about to prove that $(\star \star)$ is, in a sense, almost a necessary condition for $V$ to give a distance representation to $v$. This time we require that every circle can evolve for short time. More precisely, we require that for every circle $\gamma:[0,1] \rightarrow \mathbb{R}^{n}$ there exists $T>0$ and $\psi \in C^{2}\left([0,1] \times[0, T], \mathbb{R}^{n}\right)$ that is a $C^{2}$ embedding for every $t \in[0, T]$ such that $\psi(\cdot, 0)=\gamma$, and such that the curves $\Gamma_{t}:=\psi([0,1], t)$ move with velocity $v$ in the classical sense. Under that assumption the following theorem gives almost the converse of Theorem 5.3

THEOREM 5.4 Let $v$ be a velocity by which every circle in $\mathbb{R}^{n}$ can evolve for some time, and $V$ be a velocity. If $V$ gives a distance representation to $v$ then

$1^{\circ} \bar{v}[V] \geqslant v \geqslant \underline{v}[V]$.

$2^{\circ} v$ is a nonnegative function, nondecreasing in $k$.

$3^{\circ}$ For all $\varepsilon>0, k_{0} \geqslant 0$, all mutually orthogonal unit vectors $n_{0}, t_{0}$, and every open neighborhood $U$ of $\left(k_{0}, n_{0}, t_{0}\right)$ there exists $(\bar{k}, \bar{n}, \bar{t}) \in U \cap\left(\mathbb{R}^{+} \times \operatorname{span}\left\{n_{0}, t_{0}\right\}\right)$ such that

$$
\bar{v}[V](\bar{k}, \bar{n}, \bar{t}) \leqslant v(\bar{k}, \bar{n}, \bar{t})+\varepsilon
$$

and there exists $(\underline{k}, \underline{n}, \underline{t}) \in U \cap\left(\mathbb{R}^{+} \times \operatorname{span}\left\{n_{0}, t_{0}\right\}\right)$ such that

$$
\underline{v}[V](\underline{k}, \underline{n}, \underline{t}) \geqslant v(\underline{k}, \underline{n}, \underline{t})-\varepsilon .
$$


COROLlaRY 5.5 Let $v$ be a velocity by which every circle in $\mathbb{R}^{n}$ can evolve for short time. Let $V$ be a velocity that satisfies condition (5.1) such that $\underline{v}[V]$ and $\bar{v}[V]$ are continuous functions. Then $V$ gives a distance representation to $v$ if and only if condition $(\star \star)$ holds.

Proof of Theorem 5.4. Let $v$ be a velocity for which every circle has a short time evolution and $V$ a velocity that gives a distance representation to $v$.

Claim $1^{\circ}$. From the proof of Lemma 4.11 we see that condition $(\diamond)$ holds. By combining $(\diamond)$ and the definitions of $\bar{v}[V]$ and $\underline{v}[V]$ one obtains

$$
\begin{aligned}
& \bar{v}[V](k, n, t) \geqslant \inf _{p \in t^{\perp} \cap n^{+}} \frac{v(k, n, t) n \cdot p}{n \cdot p}=v(k, n, t), \\
& \underline{v}[V](k, n, t) \leqslant \sup _{p \in t^{\perp} \cap n^{-}} \frac{v(k, n, t) n \cdot p}{n \cdot p}=v(k, n, t) .
\end{aligned}
$$

Claim $2^{\circ}$. Assume $v(k, n, t)$ is not a nondecreasing function of $k$. Then there exists an open set $U$ such that for all $(k, n, t) \in U, \frac{\partial v}{\partial k}(k, n, t)<0$. Therefore, since $\bar{v}[V]$ is nondecreasing with respect to $k$ and $\bar{v}[V] \geqslant v$, there exists an open subset $U^{\prime}$ of $U$ and $\varepsilon>0$ such that $\bar{v}[V]>v+\varepsilon$ on $U^{\prime}$.

For $\left(k_{0}, n_{0}, t_{0}\right) \in U^{\prime}$, by assumption on the velocities considered, there exists a circle $\gamma$ : $[-L, L] \rightarrow \mathbb{R}^{n}$, parameterized by arc length, such that $\gamma^{\prime}(0)=t_{0}$ and $\gamma^{\prime \prime}(0)=k_{0} n_{0}$, and there exists $\psi \in C^{2}\left([-L, L] \times\left[0, T_{1}\right], \mathbb{R}^{n}\right)$, the motion of the curve $\gamma$ for some time $T_{1}$. Let $\Gamma_{t}:=$ $\psi([-L, L] \times\{t\})$. We now construct a perturbation of $\psi$ such that condition (b) of the definition of distance representation holds, while condition (a) fails.

Since the evolution of a planar curve by a velocity that has the direction of the curvature vector remains in the same plane, we can assume that the image of $\psi$ lies in $\mathbb{R}^{2} \times\{0\}$. Since in the argument that follows all the vectors lie in that plane, we only write their first two coordinates. In a neighborhood of $(0,0)$ one can represent the motion as the graph of a $C^{2}$ function $f:\left[-3 C_{1}, 3 C_{1}\right] \times\left[0, T_{2}\right] \rightarrow \mathbb{R}$ for some $C_{1}>0$ and $T_{2}<T_{1}$. We can assume that $f(0,0)=0, f_{x}(0,0)=0$. The curvature and the normal vector to $\Gamma_{t}$ at $(x, f(x))$ are

$$
k=\frac{\left|f_{x x}\right|}{\left(1+f_{x}^{2}\right)^{3 / 2}}, \quad p=\frac{\left(-f_{x}, 1\right)}{\sqrt{1+f_{x}^{2}}} .
$$

Near $\left(k_{0},(0,1),(1,0)\right)$, for normal vectors in the plane, the function $v(k, n, t)$ can be written as a function of only $k$ and $n_{1}$, where $n_{1}$ is the first component of $n$. More precisely let $\tilde{v}\left(k, n_{1}\right):=$ $v\left(k,\left(n_{1}, \sqrt{1-n_{1}^{2}}\right),\left(\sqrt{1-n_{1}^{2}},-n_{1}\right)\right)$. Since $f$ represents the motion by velocity $v$,

$$
f_{t}=\sqrt{1+f_{x}^{2}} \tilde{v}\left(\frac{\left|f_{x x}\right|}{\left(1+f_{x}^{2}\right)^{3 / 2}}, \frac{-f_{x}}{\sqrt{1+f_{x}^{2}}}\right) .
$$

By assumption on $v$ there exists $\delta_{1}>0$ such that if $\left(k, n_{1}\right) \in B\left(\left(k_{0}, 0\right), \delta_{1}\right)$ then $\frac{\partial \tilde{v}}{\partial k}\left(k, n_{1}\right) \leqslant 0$ and $\bar{v}[V](k, \tilde{n}, \tilde{t})>\tilde{v}\left(k, n_{1}\right)+\varepsilon$, where $\tilde{n}=\left(n_{1}, n_{2}\right)$ is a unit vector with $n_{2}>0$, and $\tilde{t}$ is a unit vector orthogonal to $\tilde{n}$. By making $C_{1}$ and $T_{2}$ smaller if necessary we can assume that on $\left[-3 C_{1}, 3 C_{1}\right] \times\left[0, T_{2}\right]$

$$
f_{x x}>k_{0} / 2,\left|f_{x}\right|<1, \quad\left(k, n_{1}\right) \in B\left(\left(k_{0}, 0\right), \delta_{1} / 2\right) \text { and } x f_{x}>0 \text { if }|x| \in\left[C_{1}, 3 C_{1}\right] .
$$


By rescaling

$$
f_{\text {new }}(x, t)=\frac{1}{C_{1}} f\left(C_{1} x, t\right), \quad \tilde{v}_{\text {new }}\left(k, n_{1}\right)=\frac{1}{C_{1}} \tilde{v}\left(\frac{k}{C_{1}}, n_{1}\right)
$$

we can also assume that $C_{1}=1$ and $f_{x x}<1$ on $[-3,3] \times\left[0, T_{2}\right]$.

To construct the perturbation we use an even cut-off function $\eta \in C^{\infty}(\mathbb{R})$ that has the following properties: $\eta(x)=1$ for $x \in[-1,1], \eta(x)=0$ if $|x|>2$ and $x \eta^{\prime}(x) \leqslant 0$ on $\mathbb{R}$. Furthermore $\|\eta\|_{C^{2}}<10$ and $\lim _{x \rightarrow 2^{-}} \eta^{\prime \prime}(x) /\left(-\eta^{\prime}(x)\right)=\infty$. Let $c:=4\left(\max _{B\left(\left(k_{0}, 1\right), \delta_{1}\right)}(|\tilde{v}|+|D \tilde{v}|)\right)$. Consider now the perturbation (with $\varepsilon_{1}>0$ to be determined)

$$
g(x, t):=f(x, t)+\varepsilon_{1} t \eta\left(\frac{x}{1+c t}\right) .
$$

Then

$$
\begin{aligned}
g_{x} & =f_{x}+\varepsilon_{1} t \eta^{\prime}\left(\frac{x}{1+c t}\right) \frac{1}{1+c t}, \\
g_{t} & =f_{t}+\varepsilon_{1} \eta\left(\frac{x}{1+c t}\right)-\varepsilon_{1} t \eta^{\prime}\left(\frac{x}{1+c t}\right) \frac{c x}{(1+c t)^{2}}, \\
\frac{g_{x x}}{\left(1+g_{x}^{2}\right)^{3 / 2}} & =\frac{f_{x x}+\frac{\varepsilon_{1} t}{(1+c t)^{2}} \eta^{\prime \prime}\left(\frac{x}{1+c t}\right)}{\left(1+f_{x}^{2}+\frac{\varepsilon_{1} t}{1+c t} \eta^{\prime}\left(\frac{x}{1+c t}\right)\left(2 f_{x}+\frac{\varepsilon_{1} t}{1+c t} \eta^{\prime}\left(\frac{x}{1+c t}\right)\right)\right)^{3 / 2}} .
\end{aligned}
$$

By continuity, using 5.6 we can now choose $\varepsilon_{1} \in(0,1)$ so that for all $(x, t) \in[-3,3] \times\left[0, T_{2}\right]$,

$$
\begin{gathered}
\left(\frac{g_{x x}}{\left(1+g_{x}^{2}\right)^{3 / 2}}, \frac{-g_{x}}{\sqrt{1+g_{x}^{2}}}\right) \in B\left(\left(k_{0}, 0\right), \delta_{1}\right), \\
\tilde{v}\left(\frac{g_{x x}}{\left(1+g_{x}^{2}\right)^{3 / 2}}, \frac{-g_{x}}{\sqrt{1+g_{x}^{2}}}\right)+\varepsilon>\frac{g_{t}}{\sqrt{1+g_{x}^{2}}} .
\end{gathered}
$$

Using the fact that $\lim _{x \rightarrow 2^{-}} \eta^{\prime \prime}(x) /\left(-\eta^{\prime}(x)\right)=\infty$ we now choose $\delta_{2} \in(0,1 / 2)$ small enough so that if $x /(1+c t) \in\left[2-2 \delta_{2}, 2\right]$ then

$$
\frac{g_{x x}}{\left(1+g_{x}^{2}\right)^{3 / 2}} \geqslant \frac{f_{x x}}{\left(1+f_{x}^{2}\right)^{3 / 2}} .
$$

It follows that for $t \in\left[0, T_{3}\right]$, where $T_{3}:=\min \left\{\delta_{2} / c, T_{2}\right\}$, the inequality 5.10 holds for all $x \in\left[-3,-2+\delta_{2}\right] \cup\left[2-\delta_{2}, 3\right]$. Note that for $t=0$ and $x \in\left[-2+\delta_{2}, 2-\delta_{2}\right]$,

$$
g_{t}-\sqrt{1+g_{x}^{2}} \tilde{v}\left(\frac{g_{x x}}{\left(1+g_{x}^{2}\right)^{3 / 2}}, \frac{-g_{x}}{\sqrt{1+g_{x}^{2}}}\right)>0 .
$$

By continuity there exists $T_{4} \in\left(0, T_{3}\right)$ such that the above holds for all $t \in\left[0, T_{4}\right]$. For $x \in$ $\left[-3,-2+\delta_{2}\right] \cup\left[2-\delta_{2}, 3\right]$, using 5.10 , we get

$$
\begin{aligned}
& g_{t}-\sqrt{1+g_{x}^{2}} \tilde{v}\left(\frac{g_{x x}}{\left(1+g_{x}^{2}\right)^{3 / 2}}, \frac{-g_{x}}{\sqrt{1+g_{x}^{2}}}\right) \\
& \quad \geqslant g_{t}-\sqrt{1+f_{x}^{2}} \tilde{v}\left(\frac{f_{x x}}{\left(1+f_{x}^{2}\right)^{3 / 2}}, \frac{-g_{x}}{\sqrt{1+g_{x}^{2}}}\right)-\frac{c}{4}\left|\sqrt{1+f_{x}^{2}}-\sqrt{1+g_{x}^{2}}\right|
\end{aligned}
$$


which by subtracting $(5.5)$ and using the definition of $c$ is

$$
\begin{aligned}
\geqslant & -\varepsilon_{1} t \eta^{\prime}\left(\frac{x}{1+c t}\right) \frac{c x}{(1+c t)^{2}}-\frac{c \sqrt{1+f_{x}^{2}}}{4}\left(\frac{-g_{x}}{\sqrt{1+g_{x}^{2}}}-\frac{-f_{x}}{\sqrt{1+f_{x}^{2}}}\right) \\
& -\frac{c}{2}\left|f_{x}-g_{x}\right| \\
\geqslant & \varepsilon_{1} t c\left|\eta^{\prime}\left(\frac{x}{1+c t}\right)\right|-c\left|f_{x}-g_{x}\right| \geqslant 0 .
\end{aligned}
$$

Combining this with (5.8) we get the following bounds on the normal velocity of the graph of $g$ :

$$
\tilde{v}\left(\frac{g_{x x}}{\left(1+g_{x}^{2}\right)^{3 / 2}}, \frac{-g_{x}}{\sqrt{1+g_{x}^{2}}}\right)+\varepsilon>\frac{g_{t}}{\sqrt{1+g_{x}^{2}}} \geqslant \tilde{v}\left(\frac{g_{x x}}{\left(1+g_{x}^{2}\right)^{3 / 2}}, \frac{-g_{x}}{\sqrt{1+g_{x}^{2}}}\right) .
$$

Let $\tilde{\psi}:[-L, L] \times\left[0, T_{4}\right] \rightarrow \mathbb{R}^{n}$ be the perturbation of $\psi$ in which the part of $\psi$ given by the graph of $\left.f\right|_{[-3,3]}$ is replaced by the graph of $g$. Let $\tilde{\Gamma}_{t}:=\tilde{\psi}([-L, L] \times\{t\})$. The normal velocity of the curves $\tilde{\Gamma}_{t}$ is equal to $v$ outside of the region given by the graph of $g$. In the region given by the graph of $g$ the curvature and tangent line are by $(5.8)$ in the region where $\bar{v}[V]>v+\varepsilon$. That, in addition to 5.12 , after a calculation like the one in the proof of Theorem 4.10, yields for some small $\tilde{\sigma}$,

$$
d_{t}(z, t)+F\left(D^{2} d(z, t), D d(z, t)\right) \geqslant 0 \quad \text { if } 0<d(z, t)<\tilde{\sigma}, t \in\left[0, T_{4}\right] .
$$

Here $d(z, t)=\operatorname{dist}\left(z, \tilde{\Gamma}_{t}\right)$. But from 5.11 it follows that the evolution of $\tilde{\Gamma}_{t}$ is not with velocity $v$; therefore $V$ does not give a distance representation to $v$.

If there is a point at which $v$ is negative, then $v$ is not nondecreasing in $k$, since $v(0, \cdot, \cdot)=0$. But we have just shown that $v$ must be nondecreasing in $k$.

Claim $3^{\circ}$. Assume that claim $3^{\circ}$ does not hold. There are two subcases with analogous proofs. Assume that there exist $\varepsilon>0, k_{0}>0$, and mutually orthogonal unit vectors $n_{0}$ and $t_{0}$ and a neighborhood $U$ of $\left(k_{0}, n_{0}, t_{0}\right)$ such that for all $(k, n, t) \in U \cap\left(\mathbb{R}^{+} \times \operatorname{span}\left\{n_{0}, t_{0}\right\}\right)$,

$$
\bar{v}[V](k, n, t)>v(k, n, t)+\varepsilon .
$$

Subcase $(i)$. There exists $(k, n, t) \in U$ such that $\frac{\partial v}{\partial k}(k, n, t)>0$. Without loss of generality, we can assume that $\frac{\partial v}{\partial k}\left(k_{0}, n_{0}, t_{0}\right)>\varepsilon_{1}>0$ and that $\frac{\partial v}{\partial k}>\varepsilon_{1} / 2$ on $U$. Let $\gamma:[-L, L] \rightarrow \mathbb{R}^{n}$ be a circle, parameterized by arc length, such that $\gamma^{\prime}(0)=t_{0}$ and $\gamma^{\prime \prime}(0)=k_{0} n_{0}$. Let $\eta(k, n, t)$ be a smooth, nonnegative function supported in $U^{\prime} \subseteq U$ such that $\eta_{k}<\varepsilon_{1} / 4$. Let $\tilde{v}=v+\eta$. Note that $\tilde{v}$ is smooth and nondecreasing in $k$ and that if we choose $U^{\prime}$ small enough then $\bar{v}[V](k, n, t)>\tilde{v}(k, n, t)+\varepsilon / 2$ for $(k, n, t) \in U \cap\left(\mathbb{R}^{+} \times \operatorname{span}\left\{n_{0}, t_{0}\right\}\right)$. By Lemma 2.4 there exist $\left\{\tilde{\Gamma}_{t}\right\}_{t \in\left[0, T_{1}\right]}$, evolution of $\gamma$ with velocity $\tilde{v}$ for some time $T_{1}>0$. Again, by a calculation like the one in [4.1), one shows that condition (b) is satisfied for $\left\{\tilde{\Gamma}_{t}\right\}_{t \in\left[0, T_{1}\right]}$, although the curve does not move with velocity $v$.

Subcase (ii). Assume that $\frac{\partial v}{\partial k}(k, n, t)=0$ for all $(k, n, t) \in U$. Then the construction of the proof of claim $2^{\circ}$ yields the desired result.

\subsection{Finding a distance representation for given velocity $v$}

The task is now to find for what velocities $v(k, n, t)$ there exists a codimension 1 velocity that gives them a distance representation. So far we have shown that if $v(k, n, t)$ has a distance representation, and all circles have a short time evolution by $v$, then 
- $v(0, n, t)=0, v$ is nonnegative and nondecreasing in $k$.

- There exists a constant $C$ such that $v(k, n, t) \leqslant C k$ for $k \geqslant 1$.

Let us call velocities $v$ that satisfy these two conditions admissible. Note that if $v$ is admissible then given a circle, if we think of $v$ as a velocity in the plane of the circle, it satisfies the conditions of Theorem 2.4. Thus every circle has a short time evolution by velocity $v$.

Recall that if $V$ gives a distance representation to $v$ then $V$ is also a barrier. If there exists a minimal barrier for velocity $v$, then, in the light of Lemma 4.9, it would be the best candidate for giving a distance representation. For a velocity $V$ to be a barrier (for velocity $v$ ) it is necessary that for every point on an arbitrary smooth hypersurface $\Upsilon$, the velocity $V$ is greater than the projections on the outward normal vector of the velocities of all the curves, contained in the interior of $\Upsilon$, that touch $\Upsilon$ at the given point. Hope that this condition is sufficient for $V$ to give a distance representation to $v$ motivates the following definition.

Given a nonnegative velocity $v$, let us define a candidate for the minimal barrier. Let $X$ be a symmetric matrix, and $p$ a unit vector. Then the matrix $(I-p \otimes p) X(I-p \otimes p)$ can be written in the form $R \operatorname{diag}\left(k_{1}, \ldots, k_{n-1}, 0\right) R^{-1}$, where $k_{1}, \ldots, k_{n-1}, 0$ are the eigenvalues and $R=\left[l_{1}, \ldots, l_{n-1}, p\right]$ is an orthogonal matrix. We then define:

- If $k_{i} \geqslant 0$ for all $i=1, \ldots, n-1$ then

$$
V[v](X, p):=\sup _{\substack{n \in p^{-} \\ t \in n^{\perp} \cap p^{\perp} \\ K \geqslant \bar{k}}} n \cdot p v\left(\frac{-K}{n \cdot p}, n, t\right) .
$$

- If $k_{i}<0$ for some $i$ then

$$
V[v](X, p):=\sup _{\substack{n \in p^{+} \\ t \in n^{\perp} \cap p^{\perp} \\ k \leqslant K \leqslant 0}} n \cdot p v\left(\frac{-K}{n \cdot p}, n, t\right),
$$

where $\bar{k}:=t^{T}(I-p \otimes p) X(I-p \otimes p) t=\sum_{i=1}^{n-1} k_{i}\left(l_{i} \cdot t\right)^{2}$.

Note that $V[v]$ is a lower semicontinuous function with values in $(-\infty, \infty]$. Also note that $V[v]$ is nonincreasing in the first argument and satisfies the condition 5.1). Let us also point out that if $v$ is nondecreasing in $k$ then it is enough to take $K=\bar{k}$ (provided that $\bar{k} \leqslant 0$ in the second equation).

LEMMA 5.6 Let $V$ be a velocity and let $v(k, n, t)$ be a nonnegative velocity such that every smooth curve in every two-dimensional plane in $\mathbb{R}^{n}$ has a short time classical evolution by velocity $v$. The following statements are equivalent:

1. $V$ is a barrier for $v$.

2. $V \geqslant V[v]$.

3. Condition $(\diamond)$ holds.

Note that if $v(k, n, t)$ is a velocity nondecreasing in $k$, then the required short time classical evolutions exist by Lemma 2.4

Proof. $(1 \Rightarrow 2) \quad$ As explained before the definition of $V[v]$, this implication is very intuitive. Let $V$ be a barrier for $v$. Assume that $V \ngtr V[v]$. Since the two cases that can occur are similar we only 
consider the first one. So we assume that there are numbers $k_{i}$ for $i=1, \ldots, n-1$, an orthogonal matrix $R=\left[l_{1}, \ldots, l_{n-1}, p\right]$, unit vectors $n$ and $t$ such that $n \cdot p<0, t \cdot n=0, t \cdot p=0$, and $K \geqslant \bar{k}=\sum_{i=1}^{n-1} k_{i}\left(l_{i} \cdot t\right)^{2}$ such that

$$
V\left(R \operatorname{diag}\left(k_{1}, \ldots, k_{n-1}, 0\right) R^{-1}, p\right)<n \cdot p v\left(\frac{-K}{n \cdot p}, n, t\right) .
$$

Let $\Upsilon_{0}$ be a smooth compact hypersurface such that $0 \in \Upsilon_{0}, p$ is the outward normal vector to $\Upsilon_{0}$ at 0 and $S=R \operatorname{diag}\left(k_{1}, \ldots, k_{n-1}, 0\right) R^{-1}$ is the matrix of the shape operator at 0 . Let $\gamma_{0}$ be a smooth curve in the plane $\operatorname{span}\{n, t\}$ such that near $0, \gamma_{0}$ is the intersection of the plane $\operatorname{span}\{n, t\}$ and $\Upsilon_{0}$. Note that the tangent vector to $\gamma_{0}$ at 0 is $t$ and that the curvature of $\gamma_{0}$ at 0 is $\frac{-1}{n \cdot p} t \cdot S t=\frac{-\bar{k}}{n \cdot p}$. Therefore there exists a curve $\gamma \in C^{\infty}\left([0, L], \mathbb{R}^{n}\right)$ in the same plane, and inside the curve $\gamma_{0}$, such that $\gamma(0)=0$ and with tangent $t$ and curvature $K$ at 0 .

Let $M:=2 \max _{\left\{x \in Y_{0}\right\}}\left\|S_{p}(x)\right\|$. By convolving $V$ with a smooth cut-off function with small support, and adding a small constant, it is easy to construct a function $\tilde{V} \in C\left(\operatorname{Sym}(n) \times S^{n-1}, \mathbb{R}\right)$ that is smooth, degenerate elliptic and if the norm of the first entry is less than $M$ then $\tilde{V}>V$, and

$$
\tilde{V}\left(R \operatorname{diag}\left(k_{1}, \ldots, k_{n-1}, 0\right) R^{-1}, p\right)<n \cdot p v\left(\frac{-K}{n \cdot p}, n, t\right) .
$$

By Lemma 2.4 there exists a classical short time evolution, $\left\{\left(\tilde{\Omega}_{t}, \tilde{\Upsilon}_{t}\right)\right\}_{t}$, of $\Upsilon_{0}$ by velocity $\tilde{V}$. Let $\left\{\left(\Omega_{t}, \Upsilon_{t}\right)\right\}_{t}$ be the generalized evolution of $\Upsilon_{0}$ by velocity $V$. Then, since $\tilde{V}>V$ on $\Upsilon_{0}$, for at least a short time $\Omega_{t} \subseteq \tilde{\Omega}_{t}$. By assumption there exists a short time classical evolution, $\left\{\Gamma_{t}\right\}_{t}$, of the curve $\gamma$. Since $V$ is a barrier for $v, \Gamma_{t} \subset \Omega_{t}$. Therefore $\Gamma_{t} \subset \tilde{\Omega}_{t}$. But then

$$
\tilde{V}\left(R \operatorname{diag}\left(k_{1}, \ldots, k_{n-1}, 0\right) R^{-1}, p\right) \geqslant n \cdot p v\left(\frac{-K}{n \cdot p}, n, t\right) .
$$

Contradiction.

$(2 \Rightarrow 3)$ Assume that $V \geqslant V[v]$. Since $k=1$ condition $(\diamond)$ now becomes

$$
\lim _{s \rightarrow 0^{+}} V\left(R \operatorname{diag}\left(\frac{-k p \cdot n}{1-s k p \cdot n}, \frac{1}{s} I_{n-2}, 0\right) R^{-1}, p\right) \geqslant v(k, n, t) n \cdot p,
$$

where $R=\left[t, l_{2}, \ldots, l_{n-1}, p\right]$. Let us consider the case $n \cdot p<0$. Since $V \geqslant V[v]$,

$$
\begin{aligned}
\lim _{s \rightarrow 0^{+}} V\left(R \operatorname{diag}\left(\frac{-k p \cdot n}{1-s k p \cdot n}, \frac{1}{s} I_{n-2}, 0\right) R^{-1}, p\right) & \\
& \geqslant \lim _{s \rightarrow 0^{+}} \sup _{\substack{\tilde{n} \in p^{-} \\
\tilde{t} \in \tilde{n}^{\perp} \cap p^{\perp}}} \tilde{n} \cdot p v\left(\frac{-\bar{k}}{\tilde{n} \cdot p}, \tilde{n}, \tilde{t}\right),
\end{aligned}
$$

where $\bar{k}=\frac{-k p \cdot n}{1-s k p \cdot n}(t \cdot \tilde{t})^{2}+\frac{1}{s}\left(1-(t \cdot \tilde{t})^{2}\right)$, and the above is

$$
\geqslant \lim _{s \rightarrow 0^{+}} n \cdot p v\left(\frac{k}{1-s k p \cdot n}, n, t\right)=v(k, n, t) n \cdot p .
$$

The case $n \cdot p>0$ is analogous.

$(3 \Rightarrow 1) \quad$ This implication was proven in Theorem 4.10 
LEMMA 5.7 Let $v$ be an admissible velocity. If $v$ depends only on $k$ or if $v(k, n, t) / k$ is a uniformly continuous function for $k \geqslant 1$ then $V[v]$ is continuous.

The proof of this lemma is straightforward, but somewhat lengthy, so we omit it. The conditions for continuity of $V[v]$ given in the lemma are not optimal, but as the next examples show, the condition given cannot be significantly improved in general.

EXAMPLES. 1. Let $\eta \in C^{\infty}(\mathbb{R})$ be a nonincreasing function such that $\eta(x)=1$ if $x \leqslant-1$ and $\eta(x)=0$ if $x \geqslant 0$. Let $v$ be a velocity in $\mathbb{R}^{3}$ given by

$$
v(k, n)=k \eta\left(k e_{3} \cdot n\right) .
$$

Then $V[v]\left(\operatorname{diag}(1,1,0), e_{3}\right)=-1$, while $V[v]\left(R \operatorname{diag}(1,1,0) R^{-1}, p\right)=0$ for $p$ near $e_{3}$ (but $\left.p \neq e_{3}\right)$ and $R$ an orthogonal matrix close to $I$ such that $R e_{3}=p$. So $V[v]$ is discontinuous at $\left(\operatorname{diag}(1,1,0), e_{3}\right)$.

2. Let $v(k, t)=k \eta\left(k e_{3} \cdot t\right)$. Then $V[v]$ is discontinuous at $\left(\operatorname{diag}(-1,-1,0), e_{3}\right)$.

THEOREM 5.8 Let $v$ be an admissible velocity.

$1^{\circ}$ If $V[v]$ is continuous and $v=\bar{v}[V[v]]$, that is,

$$
v(k, n, t)=\inf _{p \in t^{\perp} \cap n^{+}} \lim _{s \rightarrow 0^{+}} \sup _{\substack{\tilde{n} \in p^{+} \\ \tilde{t} \in \tilde{n}^{\perp} \cap p^{\perp} \\ \bar{k} \leqslant 0}} \frac{\tilde{n} \cdot p}{n \cdot p} v\left(\frac{-\bar{k}}{\tilde{n} \cdot p}, \tilde{n}, \tilde{t}\right)
$$

and $v=\underline{v}[V[v]]$, that is,

$$
v(k, n, t)=\sup _{p \in t^{\perp} \cap n^{-}} \lim _{s \rightarrow 0^{+}} \inf _{\substack{\tilde{n} \in p^{-} \\ \tilde{\tilde{n}} \in \tilde{n}^{\perp} \cap p^{\perp}}} \frac{\tilde{n} \cdot p}{n \cdot p} v\left(\frac{-\bar{k}}{\tilde{n} \cdot p}, \tilde{n}, \tilde{t}\right)
$$

where $\bar{k}=-\frac{k n \cdot p}{1-s k n \cdot p}(t \cdot \tilde{t})^{2}+\frac{1}{s}\left(1-(t \cdot \tilde{t})^{2}\right)$, then $V[v]$ gives a distance representation to $v$.

$2^{\circ}$ Regardless of whether $V[v]$ is continuous, if there exist $k_{0}, t_{0}, n_{0}, \varepsilon>0$, and a neighborhood $U$ of $\left(k_{0}, n_{0}, t_{0}\right)$ such that either

$$
\begin{array}{lll}
\bar{v}[V[v]]>v+\varepsilon & \text { on } U \cap\left(\mathbb{R}^{+} \times \operatorname{span}\left\{n_{0}, t_{0}\right\}\right) & \text { or } \\
\underline{v}[V[v]]<v-\varepsilon & \text { on } U \cap\left(\mathbb{R}^{+} \times \operatorname{span}\left\{n_{0}, t_{0}\right\}\right), &
\end{array}
$$

then there is no velocity that gives a distance representation to $v$.

Note that for any nonnegative $v, \bar{v}[V[v]] \geqslant v \geqslant \underline{v}[V[v]]$.

Proof. $1^{\circ}$ Since $V[v]$ satisfies condition $(\star \star)$, by Theorem 5.3, $V[v]$ gives a distance representation to $v$.

$2^{\circ}$ Assume that there is a velocity $V$ that gives a distance representation to $v$. Then $V$ is a barrier for $v$, and therefore by Lemma 5.6. $V \geqslant V[v]$. Therefore $\bar{v}[V] \geqslant \bar{v}[V[v]]$ and $\underline{v}[V] \leqslant \underline{v}[V[v]]$. Hence, either $\bar{v}[V]>v+\varepsilon$ on $U \cap\left(\mathbb{R}^{+} \times \operatorname{span}\left\{n_{0}, t_{0}\right\}\right)$ or $\underline{v}[V]<v-\varepsilon$ on $U \cap\left(\mathbb{R}^{+} \times \operatorname{span}\left\{n_{0}, t_{0}\right\}\right)$. Theorem 5.4 now implies that $V$ does not give a distance representation to $v$. Contradiction. 


\subsection{Classification of velocities that have a distance representation}

The obvious question is what velocities $v$ satisfy the conditions of the theorem. We present a partial answer to that question. But let us first introduce some useful objects.

Definition 5.9 Let $f:[0, \infty) \rightarrow[0, \infty)$ be a continuous function such that $f(0)=0$ and for which there exists $C$ such that if $x \geqslant 1$ then $f(x) \leqslant C x$. For $x \in[0, \infty)$ let

$$
\underline{L} f(x):=x \inf _{\alpha \in(0, x)} \frac{f(\alpha)}{\alpha}, \quad \bar{L} f(x):=x \inf _{\alpha \in(x, \infty)} \frac{f(\alpha)}{\alpha}, \quad \bar{U} f(x):=x \sup _{\alpha \in(x, \infty)} \frac{f(\alpha)}{\alpha},
$$

and for functions $f$ for which $f(x) \leqslant C x$ for all $x \geqslant 0$ let

$$
\underline{U} f(x):=x \sup _{\alpha \in(0, x)} \frac{f(\alpha)}{\alpha} .
$$

We now list some properties of the mappings $\underline{L}, \bar{L}, \bar{U}$ and $\underline{U}$. Their proofs are elementary.

LEMMA 5.10 Let $f$ be a function as in the definition above.

1. The functions $\underline{L} f, \bar{L} f, \bar{U} f$ and $\underline{U} f$ are continuous.

2. $\underline{L} f \leqslant f, \bar{L} f \leqslant f, \bar{U} f \geqslant f$ and $\underline{U} f \geqslant f$.

3. The functions $\underline{L} f(x) / x$ and $\bar{U} f(x) / x$ are nonincreasing (for $x>0$ ), while the functions $\bar{L} f(x) / x$ and $\underline{U} f(x) / x$ are nondecreasing.

4. The statements that $\underline{L} f=f$ and $\bar{U} f=f$ are both equivalent to the statement that $f(x) / x$ is a nonincreasing function. Likewise, the statements that $\underline{U} f=f$ and $\bar{L} f=f$ are both equivalent to the statement that $f(x) / x$ is a nondecreasing function.

5. $\underline{L} \bar{U} f=\bar{U} f$ and $\underline{U} \bar{L} f=\bar{L} f$ (for all $f$ in the domain of $\bar{L}$ ).

THEOREM 5.11 Velocity $v(k)$ has a distance representation if and only if $v(k)=C k$ for some nonnegative constant $C$.

Proof. Assume that $v(k)$ has a distance representation. Since $v$ is isotropic every circle has a short time classical evolution. Therefore $v$ has to be admissible. In particular there exists $C_{1}>0$ such that $v(k)<C_{1} k$ for $k \geqslant 1$. Let us compute:

$$
\begin{aligned}
\bar{v}[V[v]](k, n, t) & =\inf _{p \in t^{\perp} \cap n^{+}} \lim _{s \rightarrow 0^{+}} \sup _{\substack{\tilde{n} \in p^{+} \\
\tilde{t} \in \tilde{n}^{\perp} \cap p^{\perp} \\
\bar{k} \leqslant 0}} \frac{\tilde{n} \cdot p}{n \cdot p} v\left(\frac{k n \cdot p(\tilde{t} \cdot t)^{2}}{\tilde{n} \cdot p(1-s k n \cdot p)}-\frac{1-(\tilde{t} \cdot t)^{2}}{s \tilde{n} \cdot p}\right) \\
& =\inf _{p \in t^{\perp} \cap n^{+}} \lim _{s \rightarrow 0^{+}} \sup _{\tilde{n} \in p^{+}} \frac{\tilde{n} \cdot p}{n \cdot p} v\left(\frac{k n \cdot p}{\tilde{n} \cdot p} \frac{1}{1-s k n \cdot p}\right) \\
& =\inf _{\beta \in(0,1)} \lim _{s \rightarrow 0^{+}} \sup _{\alpha \in\left(\frac{k \beta}{1-s k \beta}, \infty\right)} \frac{k}{\alpha(1-s k \beta)} v(\alpha) \\
& =\inf _{\beta \in(0,1)} \sup _{\alpha \in(k \beta, \infty)} \frac{k}{\alpha} v(\alpha)=\underline{L} \bar{U} v(k)=\bar{U} v(k) .
\end{aligned}
$$

In a similar fashion one computes that $\underline{v}[V[v]](k, n, t)=\bar{L} v(k)$. Note that $V[v]$ is a continuous function, by Lemma 5.7. Since, by Lemma 5.10 $\bar{v}[V[v]]$ and $\underline{v}[V[v]]$ are continuous, for $v$ to have a 
distance representation it is necessary that $\bar{v}[V[v]]=v=v[V[v]]$. Therefore $\bar{L} v(k)=v=\bar{U} v(k)$. Lemma 5.10 now implies that $v(k) / k$ is both nondecreasing and nonincreasing in $k$. Therefore $v(k)=C k$ for some $C$.

On the other hand, if $v(k)=C k$ then the above computations yield $\bar{v}[V[v]]=v=\underline{v}[V[v]]$. Since $V[v]$ is continuous, Theorem 5.8 implies that $v$ has a distance representation. We should remark that a distance representation $(V[v]$ in fact) for $v(k)=C k$ was obtained first by Ambrosio and Soner [1].

To describe which velocities $v(k, t)$ have a distance representation, we introduce a couple of notions. For $x \in S^{n-1}$ let $\operatorname{dual}(x):=\left\{y \in S^{n-1}: x \cdot y=0\right\}$. For a set $A \subseteq S^{n-1}$ let $\operatorname{dual}(A):=$ $\bigcup_{x \in A} \operatorname{dual}(x)$ and $\mathcal{S}(A):=\left\{x \in S^{n-1}: \operatorname{dual}(x) \subseteq \operatorname{dual}(A)\right\}$. Note that $A \subseteq \mathcal{S}(A)$ and if $A \subseteq B$ then $\mathcal{S}(A) \subseteq \mathcal{S}(B)$. Also note that if $A$ is symmetric with respect to the origin and (or) closed, then so are dual $(A)$ and $\mathcal{S}(A)$.

Here are some examples that illustrate what the mapping $\mathcal{S}$ does. If $A$ is countable then $\mathcal{S}(A)=$ $A$. If $A$ is a circle of radius 1 then $\mathcal{S}(A)=S^{n-1}$. For $e \in S^{n-1}$ and $\alpha \in(0,1)$, if $A=\left\{x \in S^{n-1}\right.$ : $|x \cdot e|=\alpha\}$ then $\mathcal{S}(A)=\left\{x \in S^{n-1}:|x \cdot e| \geqslant \alpha\right\}$, and if $A=\left\{x \in S^{n-1}:|x \cdot e| \geqslant \alpha\right\}$ then $\mathcal{S}(A)=A$. Noting that $\mathcal{S}(A)=S^{n-1} \backslash \bigcup_{y \notin \operatorname{dual}(A)} \operatorname{dual}(y)$ also offers some insight.

On $S^{2}$ the mapping $\mathcal{S}$ is closely related to taking the convex envelope. A subset of a manifold is said to be convex if it contains all the shortest geodesics connecting any two of its points. For $A \subset S^{2}$ denote by $\mathcal{C}(A)$ its convex envelope. Let $e$ be again an arbitrary unit vector, $\tilde{A}$ a subset of $S^{2} \cap e^{+}$, and $A:=\tilde{A} \cup-\tilde{A}$. Let us prove that if $\tilde{A}$ is pathwise connected and closed then $\mathcal{S}(A)=\mathcal{C}(\tilde{A}) \cup \mathcal{C}(-\tilde{A})$.

We first show that $\mathcal{C}(\tilde{A}) \subseteq \mathcal{S}(\tilde{A})(=\mathcal{S}(A))$. Let $a, b \in \tilde{A}$ and let $c$ be on the shortest geodesic (arc of a circle) between them. It is enough to show that dual $(c) \subset \operatorname{dual}(\tilde{A})$. So let $x \in \operatorname{dual}(c)$. We can assume that $x \notin \operatorname{dual}(a) \cup \operatorname{dual}(b)$. Since $c \in \operatorname{dual}(x)$, the points $a$ and $b$ are in different components of $S^{2} \backslash \operatorname{dual}(x)$. Since $\tilde{A}$ is connected there exists $y \in \tilde{A} \cap \operatorname{dual}(x)$. Therefore $x \in$ $\operatorname{dual}(y) \subset \operatorname{dual}(\tilde{A})$.

To show the equality it suffices to show that $\mathcal{S}(\tilde{A}) \cap e^{+} \subseteq \mathcal{C}(\tilde{A})$. Assume that $x \in \mathcal{S}(\tilde{A}) \cap e^{+}$. Note that $\mathcal{S}(A)=\left\{x \in S^{2}:(\forall y \in \operatorname{dual}(x)) \operatorname{span}\{x, y\} \cap A \neq \emptyset\right\}$. We can also assume that $x \notin \tilde{A}$. Let $H=\{y \in \operatorname{dual}(x):(\exists a \in \tilde{A}) a \in \operatorname{span}\{x, y\}$ and $a \cdot y>0\}$. Since $\tilde{A}$ is closed and $x \notin \tilde{A}, H$ is closed, and since $x \in \mathcal{S}(\tilde{A}), H \cup-H=\operatorname{dual}(x)$. Therefore there exists $h \in H \cap-H$. Hence there exist $a, b \in \tilde{A} \cap \operatorname{span}\{x, h\}$ such that $a \cdot h>0$ and $b \cdot h<0$. Since $a, b$, and $x$ are all in $e^{+}$, the above implies that $x$ lies on the shortest geodesic connecting $a$ and $b$, and hence in $\mathcal{C}(\tilde{A})$.

One should note however that even if $A \subset S^{2}$ is symmetric with respect to the origin and closed, $\mathcal{S}(A)$ is not always the union of the convex envelopes of its components. To illustrate that, consider spherical coordinates on $S^{2}$ (we take latitude in $[-\pi / 2, \pi / 2]$ and longitude in $[0,2 \pi)$ ). Let $A$ be the union of the geodesics connecting $(\pi / 8,0)$ to $(\pi / 4,2 \pi / 3),(\pi / 8,2 \pi / 3)$ to $(\pi / 4,4 \pi / 3)$, and $(\pi / 8,4 \pi / 3)$ to $(\pi / 4,0)$. Then the north pole, $(\pi / 2,0)$, is in $\mathcal{S}(A)$, but not in the union of the convex envelopes of the components of $A$ (which is equal to $A$ ).

THEOREM 5.12 Let $v(k, t)$ be an admissible velocity such that $v(k, t) / k$ is uniformly continuous for $k \geqslant 1$. If velocity $v$ has a distance representation then $v(k, t)=k f(t)$ for some even function $f \in C^{\infty}\left(S^{n-1},[0, \infty)\right)$ such that the set $\mathcal{S}(\{t: f(t)=0\}) \backslash\{t: f(t)=0\}$ has empty interior. On the other hand if $v(k, t)=k f(t)$ for some smooth nonnegative even function $f$ such that $\mathcal{S}(\{t: f(t)=0\})=\{t: f(t)=0\}$ then $v$ has a distance representation, and it is given by $V[v]$.

Before we prove the theorem, let us present some examples: 
1. For any even $f \in C^{\infty}\left(S^{n-1},(0, \infty)\right), v(k, t)=k f(t)$ has a distance representation.

2. The velocity $v(k, t):=k\left(1-\left(e_{1} \cdot t\right)^{2}\right)$ has a distance representation. Here $A=\left\{e_{1},-e_{1}\right\}$.

3. The velocity $v(k, t):=k\left(e_{1} \cdot t\right)^{2}$ does not have a distance representation. For this velocity $A=\operatorname{dual}\left(e_{1}\right)$, while $\mathcal{S}(A)=S^{n-1}$.

4. Let $v(k, t):=k \eta\left(e_{1} \cdot t\right)$, where $\eta \in C^{\infty}(\mathbb{R})$ is any even, nonnegative function such that $\eta(0)>0, \eta(1)=0$, and if $b$ is the smallest positive zero of $\eta$, then $\eta(y)=0$ for all $y>b$. Note that $\mathcal{S}(A)=A=\left\{t \in S^{n-1}:\left|e_{1} \cdot t\right| \geqslant b\right\}$, so $v$ has a distance representation.

REMARK. The condition given in the theorem can be improved a bit, using the remark given before Theorem 5.3. For example, if $v(k, t)=k f(t)$ for some even smooth function $f$ such that $\mathcal{S}(\{t: f(t)=0\}) \backslash\{t: f(t)=0\}$ is nonempty, but finite, then $v$ still has a distance representation.

Proof. Let $v(k, t)$ be an admissible velocity such that $v(k, t) / k$ is uniformly continuous for $k \geqslant 1$. Assume that $v$ has a distance representation. Let us compute

$$
\bar{v}[V[v]](k, n, t)=\inf _{p \in t^{\perp} \cap n^{+}} \lim _{s \rightarrow 0^{+}} \sup _{\substack{\tilde{n} \in p^{+} \\ \tilde{t} \in \tilde{n}^{\perp} \cap p^{\perp} \\ \bar{k} \leqslant 0}} \frac{\tilde{n} \cdot p}{n \cdot p} v\left(\frac{k n \cdot p(\tilde{t} \cdot t)^{2}}{\tilde{n} \cdot p(1-s k n \cdot p)}-\frac{1-(\tilde{t} \cdot t)^{2}}{s \tilde{n} \cdot p}, \tilde{t}\right) .
$$

To do this let us show first that for fixed unit vectors $n, p, t$ such that $p \cdot t=0, n \cdot t=0$, and $n \cdot p>0$,

$$
\begin{aligned}
\lim _{s \rightarrow 0^{+}} & \sup _{\substack{\tilde{n} \in p^{+} \\
\tilde{t} \in \tilde{n}^{\perp} \cap p^{\perp} \\
k \leqslant 0}} \frac{\tilde{n} \cdot p}{n \cdot p} v\left(\frac{k n \cdot p(\tilde{t} \cdot t)^{2}}{\tilde{n} \cdot p(1-s k n \cdot p)}-\frac{1-(\tilde{t} \cdot t)^{2}}{s \tilde{n} \cdot p}, \tilde{t}\right) \\
& =\sup _{\tilde{n} \in p^{+}} \frac{\tilde{n} \cdot p}{n \cdot p} v\left(\frac{k n \cdot p}{\tilde{n} \cdot p}, t\right) .
\end{aligned}
$$

That LHS $\geqslant$ RHS follows, by taking $\tilde{t}=t$, from monotonicity of $v$. To show that LHS $\leqslant$ RHS choose a sequence $s_{i} \searrow 0$ and sequences of mutually orthogonal unit vectors $\tilde{t}_{i}$ and $\tilde{n}_{i}$ such that

$$
\text { LHS }=\lim _{i \rightarrow \infty} \frac{\tilde{n}_{i} \cdot p}{n \cdot p} v\left(\frac{k n \cdot p\left(\tilde{t}_{i} \cdot t\right)^{2}}{\tilde{n}_{i} \cdot p\left(1-s_{i} k n \cdot p\right)}-\frac{1-\left(\tilde{t}_{i} \cdot t\right)^{2}}{s_{i} \tilde{n}_{i} \cdot p}, \tilde{t}_{i}\right) .
$$

Since $v$ is even in the $t$ variable, we can assume that $\tilde{t}_{i} \cdot t \geqslant 0$ for all $i$. Note that $\left(\tilde{t}_{i} \cdot t\right)^{2} \rightarrow 1$ and hence $\tilde{t}_{i} \rightarrow t$ as $i \rightarrow \infty$. That combined with uniform continuity of $v(k, t) / k$ yields

$$
\begin{aligned}
\text { LHS } & \leqslant \lim _{i \rightarrow \infty} \frac{\tilde{n}_{i} \cdot p}{n \cdot p} v\left(\frac{k n \cdot p}{\tilde{n}_{i} \cdot p\left(1-s_{i} k n \cdot p\right)}, \tilde{t}_{i}\right) \\
& =\lim _{i \rightarrow \infty} \frac{\tilde{n}_{i} \cdot p}{n \cdot p} v\left(\frac{k n \cdot p}{\tilde{n}_{i} \cdot p}, \tilde{t}_{i}\right) \leqslant \text { RHS. }
\end{aligned}
$$

Therefore

$$
\begin{aligned}
\bar{v}[V[v]](k, n, t) & =\inf _{p \in t^{\perp} \cap n^{+}} \sup _{\tilde{n} \in p^{+}} \frac{\tilde{n} \cdot p}{n \cdot p} v\left(\frac{k n \cdot p}{\tilde{n} \cdot p}, t\right) \\
& =\inf _{\beta \in(0,1)} \sup _{\alpha \in(k \beta, \infty)} \frac{k}{\alpha} v(\alpha, t)=\underline{L} \bar{U} v(k, t)=\bar{U} v(k, t) .
\end{aligned}
$$


Note that uniform continuity of $v(k, t) / k$ for $k \geqslant 1$ implies that $\bar{U} v(k, t)$ is continuous. Since $\bar{v}[V[v]]$ is continuous, Theorem 5.8 implies that $v=\bar{v}[V[v]]$.

Since $v=\bar{U} v$ the function $v(k, t) / k$ is nonincreasing in $k$ for every fixed $t$. That enables us to define $\bar{w}(t):=\lim _{k \rightarrow \infty} v(k, t) / k$. Uniform continuity of $v(k, t) / k$ implies that $\bar{w}$ is continuous.

Let $A:=\left\{t \in S^{n-1}: \bar{w}(t)=0\right\}$. We now compute $\underline{v}[V[v]](k, n, t)$ :

$$
\underline{v}[V[v]](k, n, t)=\sup _{p \in t^{\perp} \cap n^{-}} \lim _{s \rightarrow 0^{+}} \inf _{\substack{\tilde{n} \in p^{-} \\ \tilde{t} \in \tilde{n}^{\perp} \cap \tilde{p}}} \frac{\tilde{n} \cdot p}{n \cdot p} v\left(\frac{k n \cdot p(\tilde{t} \cdot t)^{2}}{\tilde{n} \cdot p(1+s k|n \cdot p|)}+\frac{1-(\tilde{t} \cdot t)^{2}}{s|\tilde{n} \cdot p|}, \tilde{t}\right) .
$$

For fixed $k, n, t$ and $p$ such that $n \cdot t=0, p \cdot t=0$ and $n \cdot p<0$ there are two distinct cases:

Case (i). Assume that $p \in \operatorname{dual}(A)$. Then there exists $\tilde{t}(p)$ orthogonal to $p$ such that $\bar{w}(\tilde{t}(p))=0$. Hence

$$
\begin{aligned}
\lim _{s \rightarrow 0^{+}} \inf _{\substack{\tilde{n} \in p^{-} \\
\tilde{t} \in \tilde{n}^{\perp} \cap p^{\perp}}} & \frac{\tilde{n} \cdot p}{n \cdot p} v\left(\frac{k n \cdot p(\tilde{t} \cdot t)^{2}}{\tilde{n} \cdot p(1+s k|n \cdot p|)}+\frac{1-(\tilde{t} \cdot t)^{2}}{s|\tilde{n} \cdot p|}, \tilde{t}\right) \\
\leqslant & \lim _{s \rightarrow 0^{+}} \inf _{\tilde{n} \in \tilde{t}(p)^{\perp} \cap p^{-}} \frac{|\tilde{n} \cdot p|}{|n \cdot p|} v\left(\frac{k|n \cdot p|(\tilde{t}(p) \cdot t)^{2}}{|\tilde{n} \cdot p|(1+s k|n \cdot p|)}+\frac{1-(\tilde{t}(p) \cdot t)^{2}}{s|\tilde{n} \cdot p|}, \tilde{t}(p)\right) \\
\leqslant & \lim _{s \rightarrow 0^{+}} \lim _{\alpha \rightarrow 0^{+}} \frac{\alpha}{|n \cdot p|} v\left(\left(\frac{k|n \cdot p|(\tilde{t}(p) \cdot t)^{2}}{(1+s k|n \cdot p|)}+\frac{1-(\tilde{t}(p) \cdot t)^{2}}{s}\right) \frac{1}{\alpha}, \tilde{t}(p)\right) \\
= & 0 .
\end{aligned}
$$

Case (ii). Assume that $p \notin \operatorname{dual}(A)$, which is equivalent to $\operatorname{dual}(p) \cap A=\emptyset$. Let $m(p):=$ $\min \{\bar{w}(x): x \in \operatorname{dual}(p)\}$. Note that $m(p)>0$, since $\bar{w}$ is continuous, dual $(p)$ compact, and $\operatorname{dual}(p) \cap A=\emptyset$. We claim that

$$
\lim _{s \rightarrow 0^{+}} \inf _{\substack{\tilde{n} \in p^{-} \\ \tilde{t} \in \tilde{n}^{\perp} \cap p^{\perp}}} \frac{\tilde{n} \cdot p}{n \cdot p} v\left(\frac{k n \cdot p(\tilde{t} \cdot t)^{2}}{\tilde{n} \cdot p(1+s k|n \cdot p|)}+\frac{1-(\tilde{t} \cdot t)^{2}}{s|\tilde{n} \cdot p|}, \tilde{t}\right)=\inf _{\tilde{n} \in t^{\perp} \cap p^{-}} \frac{|\tilde{n} \cdot p|}{|n \cdot p|} v\left(\frac{k|n \cdot p|}{|\tilde{n} \cdot p|}, t\right) .
$$

We argue as when proving 5.3. That LHS $\leqslant$ RHS is established by taking $\tilde{t}=t$. To show that LHS $\geqslant$ RHS we consider sequences $s_{i} \searrow 0, \tilde{n}_{i}$, and $\tilde{t}_{i}$ such that $\tilde{n}_{i} \cdot p<0, \tilde{n}_{i} \cdot \tilde{t}_{i}=0, \tilde{t}_{i} \cdot p=0$, and

$$
\text { LHS }=\lim _{i \rightarrow \infty} \frac{\tilde{n}_{i} \cdot p}{n \cdot p} v\left(\frac{k n \cdot p\left(\tilde{t}_{i} \cdot t\right)^{2}}{\tilde{n}_{i} \cdot p\left(1+s_{i} k|n \cdot p|\right)}+\frac{1-\left(\tilde{t}_{i} \cdot t\right)^{2}}{s_{i}\left|\tilde{n}_{i} \cdot p\right|}, \tilde{t}_{i}\right) .
$$

We can assume that $t \cdot \tilde{t}_{i} \geqslant 0$ for all $i$. Note that $m(p)>0$, and $v(k, t) / k$ nonincreasing in $k$, imply that for all $x$ in $\operatorname{dual}(p), v(k, x) / k>m(p)$ for all $k>0$. Therefore $\left(1-\left(\tilde{t}_{i} \cdot t\right)^{2}\right) / s_{i}$ must be bounded. Consequently, $\tilde{t}_{i} \rightarrow t$ as $i \rightarrow \infty$. The remainder of the argument is as in 5.3.

We now also split finding $\underline{v}[V[v]](k, n, t)$ into two cases:

Case $1^{\circ}$. Assume that $t \in \mathcal{S}(A)$. Then all unit vectors $p$ orthogonal to $t$ are in $\operatorname{dual}(A)$. Therefore case (i) implies that $\underline{v}[V[v]](k, n, t)=0$. 
Case $2^{\circ}$. Assume that $t \notin \mathcal{S}(A)$, which is equivalent to assuming that $\operatorname{dual}(A) \backslash \operatorname{dual}(t) \neq \emptyset$. Let $P:=\operatorname{dual}(A) \backslash \operatorname{dual}(t)$. Cases (i) and (ii) combined now yield

$$
\begin{aligned}
\underline{v}[V[v]](k, n, t) & =\sup _{p \in P \cap n^{-}} \inf _{\tilde{n} \in t^{\perp} \cap p^{-}} \frac{|\tilde{n} \cdot p|}{|n \cdot p|} v\left(\frac{k|n \cdot p|}{|\tilde{n} \cdot p|}, t\right) \\
& =\sup _{p \in P \cap n^{-}} \inf _{\alpha \in(k|n \cdot p|, \infty)} \frac{k}{\alpha} v(\alpha, t) \\
& \leqslant \sup _{p \in P \cap n^{-}} \inf _{\alpha \in(k, \infty)} \frac{k}{\alpha} v(\alpha, t)=\bar{L} v(k, t) .
\end{aligned}
$$

In both cases $\underline{v}[V[v]](k, n, t) \leqslant \bar{L} v(k, t) \leqslant v(k, t)$. Note that $\bar{L} v(k, t)$ is continuous. Therefore, if $v$ were greater than $\bar{L} v$ for some $(k, t)$, then $v$ would be greater than $\underline{v}[V[v]]+\varepsilon$, for some $\varepsilon>0$, in a neighborhood of $(k, n, t)$, where $n$ is any unit vector orthogonal to $t$. But that would contradict Theorem 5.8 .

Therefore $v=\bar{L} v$. Since we already know that $v=\bar{U} v$, we conclude, as in Theorem 5.11 that $v$ is linear in $k$ for every fixed $t$. That is, there exists a nonnegative function $f$ such that $v(k, t)=$ $k f(t)$. Since $v$ is smooth, $f$ must be smooth too.

Note that $A=\{t: f(t)=0\}$. Cases $1^{\circ}$ and $2^{\circ}$ now imply that

$$
\underline{v}[V[v]](k, t)= \begin{cases}0 & \text { if } t \in \mathcal{S}(A), \\ k f(t) & \text { otherwise. }\end{cases}
$$

Assume that $\mathcal{S}(A) \backslash A$ has nonempty interior. Let $t_{0}$ be in the interior. Then in a neighborhood of $\left(1, t_{0}\right), v$ is strictly greater than 0 , while $\underline{v}[V[v]]$ is equal to 0 . Theorem 5.8 then implies that $v$ does not have a distance representation, which contradicts the assumption we made. Therefore $\mathcal{S}(A) \backslash A$ must have empty interior.

If, on the other hand, $v(k, t)=k f(t)$ for some smooth nonnegative even function $f$ such that $\mathcal{S}(\{t: f(t)=0\})=\{t: f(t)=0\}$, then the calculations above show that $\bar{v}[V[v]]=v$ and $\underline{v}[V[v]]=v$. Hence, by Theorem 5.8, $V[v]$ gives a distance representation to $v$.

To compute $V[v]$ for the velocities of the form $v(k, t)=k f(t)$ one can use the definition of $V[v]$ that now becomes: for real numbers $k_{i}, i=1, \ldots, n-1$, and an orthogonal matrix $R=\left[l_{1}, \ldots, l_{n-1}, p\right]$,

$$
V[v]\left(R \operatorname{diag}\left(k_{1}, \ldots, k_{n-1}, 0\right) R^{-1}, p\right):=\max _{t \in p^{\perp}}-f(t) \sum_{i=1}^{n-1} k_{i}\left(l_{i} \cdot t\right)^{2} .
$$

For many velocities of the form $v(k, t)=k f(t)$ for which $V[v]$ gives a distance representation to $v$, it is an open problem to determine whether $V[v]$ surrounds $v$.

Proposition 5.13 Let $v$ be a velocity of the form $v(k, n)=k f(n)$ where $f \in C^{\infty}\left(S^{n-1}\right.$, $[0, \infty)$ ). Then $v$ has a distance representation if and only if $f$ is a constant.

Proof. Using the techniques of the previous two theorems one shows that

$$
\begin{aligned}
& \bar{v}[V[v]](k, n)=\inf _{p \in n^{+}} \sup _{\tilde{n} \in p^{+}} k f(\tilde{n}), \\
& \underline{v}[V[v]](k, n)=\sup _{p \in n^{-}} \inf _{\tilde{n} \in p^{-}} k f(\tilde{n}) .
\end{aligned}
$$


Assume that $v$ has a distance representation. Note that $\bar{v}[V[v]]$ and $\underline{v}[V[v]]$ are continuous. Theorem 5.8 then implies that $v=\bar{v}[V[v]]=\underline{v}[V[v]]$. Let $m$ be the global minimum of $f$, and $n_{m}$ a point for which $f\left(n_{m}\right)=m$, and let $M$ be the global maximum of $f$, and $n_{M}$ a point where it is reached.

Assume that $f$ is not a constant. Then there exists $n_{0}$ such that $m<f\left(n_{0}\right)<M$. There are two cases: If $f\left(-n_{0}\right)>m$, then

$$
v\left(1, n_{m}\right) \geqslant \inf _{p \in n_{m}^{+}} \min \left\{f\left(n_{0}\right), f\left(-n_{0}\right)\right\}>m,
$$

which contradicts $v\left(1, n_{m}\right)=m$. On the other hand, if $f\left(-n_{0}\right)<M$ then

$$
v\left(1, n_{M}\right) \leqslant \sup _{p \in n_{M}^{-}} \max \left\{f\left(n_{0}\right), f\left(-n_{0}\right)\right\}<M .
$$

Contradiction. Therefore $f$ must be a constant.

If $f$ is a constant, then $v$ has a distance representation by Theorem 5.11 .

\section{Some remarks on barriers of De Giorgi}

Barriers were introduced by De Giorgi [10] as an abstract way to define weak (generalized) evolution for a wide range of velocities. The notion of barrier that we have used in the previous sections is not the same, but is very much related to the barriers that we are now about to study. Following Bellettini and Novaga [5] (and references therein), let us introduce the barriers of De Giorgi and related objects. Let $\mathcal{F}$ be a family of mappings from closed intervals, with nonnegative endpoints, into subsets of $\mathbb{R}^{n}$. In the applications, given a smooth codimension $n-k>1$ velocity $v$, we denote by $\mathcal{F}_{v}$ the set of all classical evolutions. Given a lower semicontinuous codimension 1 velocity $V$ we denote by $\mathcal{F}_{V}^{\bar{V}}$ the set of all classical evolutions by velocity $V$ with their interiors, or to be more precise, if $\left\{\left(\Omega_{t}, \Upsilon_{t}\right)\right\}_{t \in[a, b]}$ is an evolution (flow) by velocity $V$, then the mapping $t \mapsto \Omega_{t} \cup \Upsilon_{t}$ is in $\mathcal{F}_{V}=$. By $\mathcal{F}_{V}^{\leqslant}$and $\mathcal{F}_{V}^{<}$we denote respectively the set of all smooth subflows and the set of all smooth strict subflows (meaning that in Definition 2.1 the equality is replaced by $\frac{\partial \psi}{\partial t} \cdot n<V(D n, n)$ ). A mapping $\phi:[0, \infty) \rightarrow \mathcal{P}\left(\mathbb{R}^{n}\right)$ is called a barrier for $\mathcal{F}$ if for all $f:[a, b] \rightarrow \mathcal{P}\left(\mathbb{R}^{n}\right)$ with $f \in \mathcal{F}$ the condition $f(a) \subseteq \phi(a)$ implies $f(s) \subseteq \phi(s)$ for all $s \in[a, b]$. We denote the set of all barriers for given $\mathcal{F}$ by $\mathcal{B}(\mathcal{F})$. For $E$ a subset of $\mathbb{R}^{n}$, we define the minimal barrier by

$$
\mathcal{M}(E, \mathcal{F})(t):=\bigcap\{\phi(t) \in \mathcal{B}(\mathcal{F}): E \subseteq \phi(0)\} .
$$

We also define the regularized minimal barrier by

$$
\mathcal{M}^{*}(E, \mathcal{F})(t):=\bigcap_{\rho>0} \mathcal{M}(E+\rho, \mathcal{F})(t) .
$$

For a barrier $\phi$ we define $\chi \phi(x, t):=1-\mathbb{1}_{\phi(t)}(x)$ and

$$
\underline{\phi}(t):=\bigcup_{\varepsilon>0} \bigcap_{s \in(0 \vee(t-\varepsilon), t+\varepsilon)}(\phi(s)-\varepsilon),
$$

where for a set $A$ the set $A-\varepsilon:=\left\{x \in \mathbb{R}^{n}: \operatorname{dist}\left(x, \mathbb{R}^{n} \backslash A\right)>\varepsilon\right\}$. Note that $\underline{\phi}(t) \subseteq \phi(t)$ for all $t$. We claim that

$$
(\chi \phi)^{*}=\chi \underline{\phi} .
$$


To prove that claim it is enough to show that $(\chi \phi)^{*}=0$ exactly when $\chi \phi=0$. By the definition of upper semicontinuous envelope, $(\chi \phi)^{*}(x, t)=0$ iff there exists an $\varepsilon>0$ such that $\chi \phi=0$ on $(0 \vee(t-\varepsilon), t+\varepsilon) \times B(x, \varepsilon)$. That is equivalent to saying that $x \in \bigcap_{s \in(0 \vee(t-\varepsilon), t+\varepsilon)}(\phi(s)-\varepsilon)$ for some $\varepsilon>0$, which is equivalent to $\chi \phi(x, t)=0$. A consequence of this claim is that $\phi(t)$ is open for all $t$.

For a set $A$ and vector $x$ denote by $\tau_{x}(A)$ the translation of $A$ by $x$. The following lemma seems to state the obvious; however, due to the fact that we have no estimates on the interval of existence of short time evolutions by the given velocity, its proof is not trivial.

LEMMA 6.1 Let $V(D n, n)$ be a smooth codimension 1 velocity that may not be nondecreasing in the $D n$ argument, but for which for every smooth compact hypersurface without boundary embedded in $\mathbb{R}^{n}$ there exists a short time classical evolution. Let $\phi$ be an open barrier in $\mathcal{B}\left(\mathcal{F}_{V}^{\bar{V}}\right)$. Then $\phi$ is in $\mathcal{B}\left(\mathcal{F}_{V}^{<}\right)$.

Proof. Let $\left\{\left(\Omega_{t}, \Upsilon_{t}\right)\right\}_{t \in[a, b]}$ be a smooth strict subflow by velocity $V$ such that $\Omega_{a} \cup \Upsilon_{a} \subset \phi(a)$. Let $d(t):=\operatorname{dist}\left(\Omega_{t} \cup \Upsilon_{t}, \partial \phi(t)\right)$. Note that $d(a)>0$. Therefore it suffices to prove that $d(t) \geqslant d(a)$ for all $t \in[a, b]$. Note that if $\left\{\left(\Omega_{t}, \Upsilon_{t}\right)\right\}_{t}$ were a classical evolution then it is an easy consequence of translation invariance of the evolution that $d(t)$ is nondecreasing.

Let $A:=\{t \in(a, b]: d(t)<d(a)\}$. Our goal is to show that $A$ is empty. Assume it is not. Then we can consider $t_{0}:=\inf A$. There are two cases:

Case $1^{\circ}$. Assume that $t_{0} \notin A$, that is, $d\left(t_{0}\right) \geqslant d(a)$. By assumptions on $V$ there exists $\varepsilon>0$ and a classical evolution $\left\{\left(\tilde{\Omega}_{t}, \tilde{\Upsilon}_{t}\right)\right\}_{t \in\left[t_{0}, t_{0}+\varepsilon\right]}$ of $\left(\Omega_{t_{0}}, \Upsilon_{t_{0}}\right)$ for $t \in\left[t_{0}, t_{0}+\varepsilon\right)$. By making $\varepsilon$ smaller if necessary, since $\left(\Omega_{t}, \Upsilon_{t}\right)$ is a strict subflow, we have $\Omega_{t} \cup \Upsilon_{t} \subset \tilde{\Omega}_{t} \cup \tilde{\Upsilon}_{t}$ for $t \in\left[t_{0}, t_{0}+\varepsilon\right)$. Therefore $d(t)>\operatorname{dist}\left(\tilde{\Omega}_{t} \cup \tilde{r}_{t}, \partial \phi(t)\right) \geqslant d\left(t_{0}\right) \geqslant d(a)$ for $t \in\left(t_{0}, t_{0}+\varepsilon\right)$. Therefore $\left[t_{0}, t_{0}+\varepsilon\right) \cap A=\emptyset$, which implies that $t_{0} \neq \inf A$. Contradiction.

Case $2^{\circ}$. Assume that $t_{0} \in A$. Then $d\left(t_{0}\right)<d(a)$. Since $\Upsilon_{t_{0}}$ is compact and $\partial \phi\left(t_{0}\right)$ is closed, there exist $x_{0} \in \Upsilon_{t_{0}}$ and $y_{0} \in \partial \phi\left(t_{0}\right)$ such that $d\left(t_{0}\right)=\operatorname{dist}\left(x_{0}, y_{0}\right)$. Since $\left\{\left(\Omega_{t}, \Upsilon_{t}\right)\right\}_{t}$ is a continuous evolution and $d(t) \geqslant d(a)$ for all $t \in\left[a, t_{0}\right)$, there exists $\delta>0$ such that $B\left(y_{0}, \delta\right) \subset \phi(t)$ for all $t \in\left(t_{0}-\delta, t_{0}\right)$. Let $\tilde{\Upsilon}_{0}$ be the sphere of radius $\delta / 3$ centered at $y_{0}$. By assumption on $V$ there exists $\varepsilon_{1}>0$ such that there exists a classical evolution $\left\{\left(\tilde{\Omega}_{t}, \tilde{\Upsilon}_{t}\right)\right\}_{t \in[0, \varepsilon)}$ of $\tilde{\Upsilon}_{0}$ by velocity $V$. By making $\varepsilon$ smaller if necessary we can assume that for all $t \in[0, \varepsilon), \tilde{\Omega}_{t} \cup \tilde{\Upsilon}_{t} \subset B\left(y_{0}, \delta / 2\right)$. Let

$$
U:=\bigcup\left\{\tau_{y}\left(\tilde{\Omega}_{\varepsilon / 2} \cup \tilde{\Upsilon}_{\varepsilon / 2}\right):|y| \leqslant \delta / 2\right\}
$$

Note that $U$ is nothing else than the union of all classical evolutions at time $t_{0}$ of spheres centered at $y_{0}+y$ of radius $\delta / 3$ beginning at time $t_{0}-\varepsilon / 2$. Since all these spheres are at time $t_{0}-\varepsilon / 2$ in $\phi\left(t_{0}-\varepsilon / 2\right)$, we have $U \subseteq \phi\left(t_{0}\right)$. But $y_{0} \in U$, which contradicts the fact that $y_{0} \in \partial \phi\left(t_{0}\right)$.

Therefore $A$ must be empty.

Note that one can use this proof to show that $d(t)$ is a nondecreasing function. Also recall that it was proven in Proposition 5.2 of [5] that if $V(D n, n)$ is a lower semicontinuous velocity nonincreasing in $D n$ and $\phi \in \mathcal{B}\left(\mathcal{F}_{V}^{<}\right)$then $\chi \phi$ is a viscosity subsolution of the level-set equation (that corresponds to $V$ ). The proof in [5] is carried out for equations for which the standard viscosity solutions are applicable. The extension of this result (and of several others that we will quote) to a larger class of equations, using the viscosity solutions of Ishii and Souganidis, is straightforward. 
LEMMA 6.2 Let $v(k, n, t)$ be a velocity by which every circle can evolve for a short time and $V$ a codimension 1 velocity. If $\phi$ is a barrier for $\mathcal{F}_{v}\left(\right.$ or $\left.\mathcal{F}_{V}^{<}\right)$then $\phi(0)$ is equal to the interior of $\phi(0)$, and $\underline{\phi}$ is a barrier for $\mathcal{F}_{v}$ (resp. $\mathcal{F}_{V}^{<}$).

Proof. This lemma follows from translation invariance of the evolutions by velocities $v$ and $V$. Let $x_{0}$ be a point in the interior of $\phi(0)$. There exists a circle $\Gamma_{0}$ such that $x_{0} \in \Gamma_{0} \subset \phi(0)$. There exists $\delta>0$ such that $\bigcup_{y \in B(0,2 \delta)} \tau_{y}\left(\Gamma_{0}\right)$ is a subset of the interior of $\phi(0)$. Let $\left\{\Gamma_{t}\right\}_{t \in[0, b]}$ be the evolution of the circle by velocity $v$ for some time $b>0$. Let $d(t):=\operatorname{dist}\left(x_{0}, \Gamma_{t}\right)$. Note that $d$ is continuous and $d(0)=0$. Therefore there exists $\varepsilon>0$ such that for all $t<\varepsilon, d(t)<\delta$. We can assume that $\varepsilon<\delta$. Then

$$
B\left(x_{0}, \varepsilon\right) \times[0, \varepsilon) \subseteq \bigcup_{y \in B(0,2 \delta), t \in[0, \varepsilon)} \tau_{y}\left(\Gamma_{t}\right) \times\{t\} \subset \bigcup_{t \in[0, \varepsilon)} \phi(t) \times\{t\} .
$$

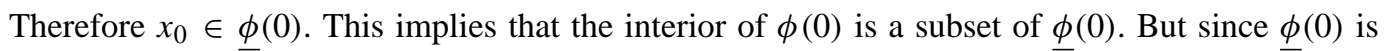
open and a subset of $\phi(0)$ we conclude that $\phi(0)=$ int $\phi(0)$.

To prove the second claim let $\left\{\Gamma_{t}\right\}_{t \in[a, b]}$ be a classical evolution by velocity $v$. Assume that $\Gamma_{a} \subseteq \underline{\phi}(a), s \in(a, b]$, and let $x_{0} \in \Gamma_{s}$. It is enough to show that for some $\varepsilon>0, B\left(x_{0}, \varepsilon\right) \times$ $(s-\varepsilon, \bar{s}+\varepsilon) \subset \bigcup_{t \in(s-\varepsilon, s+\varepsilon)} \phi(s) \times\{t\}$. The construction is very similar to the one above, so we omit it.

The claim for $V$ was proven in Proposition 4.1 of [5]. It can also be proven analogously to the proof above.

\subsection{Comparing minimal barriers for $v$ and $V[v]$}

LEMMA 6.3 Let $v(k, n, t)$ be a nonnegative velocity. Then for a bounded set $E$,

$$
\mathcal{M}^{*}\left(E, \mathcal{F}_{v}\right)(t) \subseteq \mathcal{M}^{*}\left(E, \mathcal{F}_{V[v]}^{\leqslant}\right)(t)
$$

Proof. For fixed $\rho>0$ let $\phi(t):=\mathcal{M}\left(E+\rho, \mathcal{F}_{V[v]}^{\leqslant}\right)(t)$. Note that $\phi(t)$ is open and $\phi(t)=\underline{\phi}(t)$. It is enough to show that $\phi(t)$ is a barrier for $\mathcal{F}_{v}$. Let now $\left\{\Gamma_{t}\right\}_{t \in[a, b]}$ be a classical evolution by velocity $v$ such that $\Gamma_{a} \subset \phi(a)$. Let $s \in(a, b]$. Since $\Gamma_{a}$ is closed while $\phi(a)$ is open, there exists $\delta>0$ such that $\Gamma_{a}+2 \delta \subset \phi(a)$. By Lemma 3.2 there exists $\sigma>0$ such that $d(x, s):=\operatorname{dist}\left(x, \Gamma_{t}\right)$ is a $C^{2}$ function on $A:=\{(x, t): d(x, t) \in(0, \sigma)\}$. We can assume that $2 \delta<\sigma$. Note that the function $V[v]$ (although possibly not continuous) satisfies condition $(\diamond)$. The calculation 4.1 then shows that

$$
d_{t}+V[v]\left(D^{2} d, D d\right) \geqslant 0 \quad \text { on } A .
$$

Let $\Omega_{s}:=\Gamma_{s}+\delta=\{d(\cdot, s)<\delta\}$ and $\Upsilon_{s}:=\partial \Omega_{s}=\{d(\cdot, s)=\delta\}$. Let $d_{ \pm}(\cdot, s)$ be the signed distance to $\Upsilon_{s}$. Note that $d_{ \pm}=d-\delta$, therefore for all $s \in[a, b]$,

$$
\frac{\partial d_{ \pm}}{\partial t}+V[v]\left(D^{2} d_{ \pm}, D d_{ \pm}\right) \geqslant 0 \quad \text { on } \Upsilon_{s}
$$

Consequently, $\Upsilon_{t}$ is a classical subflow by velocity $V[v]$. Since $\phi$ is an open barrier for $V[v]$, for $s \in(a, b]$ we have $\Omega_{s} \cup \Upsilon_{s} \subset \phi(s)$. Therefore $\Gamma_{s} \subset \phi(s)$.

The following corollary gives a criterion for when $\mathcal{M}^{*}\left(\Gamma_{0}, \mathcal{F}_{V[v]}^{\leqslant}\right)(t)$ does not capture the evolution by velocity $v$ : 
COROLlARY 6.4 Let $v(k, n, t)$ be a nonnegative velocity by which every circle can evolve for a short time. If $v$ is not nonincreasing in $k$ or there exist $k_{0}, t_{0}, n_{0}, \varepsilon>0$, and a neighborhood $U$ of $\left(k_{0}, n_{0}, t_{0}\right)$ such that either $\bar{v}[V[v]]>v+\varepsilon$ on $U \cap\left(\mathbb{R}^{+} \times \operatorname{span}\left\{n_{0}, t_{0}\right\}\right)$, or $\underline{v}[V[v]]<v-\varepsilon$ on $U \cap\left(\mathbb{R}^{+} \times \operatorname{span}\left\{n_{0}, t_{0}\right\}\right)$, then there exists a time $T>0$, a smooth curve $\Gamma_{0}$, and its short time classical evolution $\left\{\Gamma_{t}\right\}_{t \in[0, T]}$ such that for all $t \in(0, T]$,

$$
\Gamma_{t} \varsubsetneqq \mathcal{M}^{*}\left(\Gamma_{0}, \mathcal{F}_{V[v]}^{\leqslant}\right)(t) .
$$

Proof. Let $v$ be a velocity that satisfies the assumptions. If $v$ is not nondecreasing in $k$ then let $\Gamma_{0}$ be the circle $\gamma$ from case $2^{\circ}$ of the proof of Theorem 5.4, where $V=V[v]$. Let $\tilde{\Gamma}_{t}$ and $\tilde{\sigma}$ be from the same case. It was shown that for some $T>0$,

$$
d_{t}(z, t)+V[v]\left(D^{2} d(z, t), D d(z, t)\right) \geqslant 0 \quad \text { if } d(z, t) \in(0, \tilde{\sigma}) \text { and } t \in[0, T],
$$

where $d(z, t):=\operatorname{dist}\left(z, \tilde{\Gamma}_{t}\right)$. Note that $\tilde{\Gamma}_{t} \subset \mathcal{M}^{*}\left(\Gamma_{0}, \mathcal{F}_{V[v]}\right)(t)$. The proof is analogous to showing that $\Gamma_{s} \subset \phi(s)$ in the previous lemma.

If $v$ is nondecreasing in $k$ then let $\gamma$ and $\tilde{\Gamma}_{t}$ be as in case $3^{\circ}$ of the proof of Theorem 5.4. The rest of the argument is the same as above.

In both cases the fact that $\Gamma_{t} \subset \mathcal{M}^{*}\left(\Gamma_{0}, \mathcal{F}_{V[v]}\right)(t)$ follows from the lemma above.

The following theorem proves a generalization of De Giorgi's conjecture. If $V[v]$ is continuous we show that the minimal barrier for $v$ is the same as the minimal barrier for $V[v]$, which is the same as the zero-level set of the solution of the level-set equation. A consequence is that $V[v]$ is continuous and if there is no velocity that surrounds $v$ (which is the same as saying that $V[v]$ does not surround $v$ ) then the barriers do not capture the motion either. That is, there are curves whose classical evolution by the given velocity is a proper subset of either of the regularized minimal barriers.

THEOREM 6.5 Let $v$ be an admissible velocity and $E$ a bounded set. If $V[v]$ is continuous then

$$
\mathcal{M}^{*}\left(E, \mathcal{F}_{V[v]}^{\leqslant}\right)(t)=\mathcal{M}^{*}\left(E, \mathcal{F}_{v}\right)(t)=\{x: u(x, t)=0\},
$$

where $u$ is the unique viscosity solution of the level-set equation

$$
\begin{aligned}
& u_{t}+F\left(D^{2} u, D u\right)=0, \\
& u(x, 0)=\operatorname{dist}(x, E) .
\end{aligned}
$$

Proof. The equality $\mathcal{M}^{*}\left(E, \mathcal{F}_{V[v]}^{\leqslant}\right)(t)=\{x: u(x, t)=0\}$ follows from Corollary 6.1 of [5].

Lemma 6.3 implies that it is enough to prove that $\mathcal{M}^{*}\left(E, \mathcal{F}_{V[v]}^{\leqslant}\right)(t) \subseteq \mathcal{M}^{*}\left(E, \mathcal{F}_{v}\right)(t)$. Since $E+\rho$ is open for every $\rho>0$, it is enough to show that if $\phi$ is a barrier for $\mathcal{F}_{v}$ then $\phi(t)$ is a barrier for $\mathcal{F}_{V[v]}^{\leqslant}$. Since sublevel sets of subsolutions of the level-set equation are barriers (see Theorem 3.2 in [6], for example), it suffices to show that $\chi \phi(t)$ is a viscosity subsolution of the level-set equation. Note that, since $v$ is admissible, by definition of $V$ there exists $C>0$ such that $v(k, n, t) \leqslant C k$ if $k \geqslant 1$ and $V[v](X, p)<C(\|X\|+1)$. Therefore $F_{*}(0,0)=F^{*}(0,0)=0$. Let $\psi$ be an admissible test function such that $\chi \underline{\phi}-\psi$ has a maximum at $\left(x_{0}, t_{0}\right)$. There are four cases:

Case $1^{\circ}$. If $\chi \phi\left(x_{0}, t_{0}\right)=0$ then (since $\chi \phi$ is upper semicontinuous) $\chi \phi=0$ in a neighborhood of $\left(x_{0}, t_{0}\right)$. Therefore $D \psi\left(x_{0}, t_{0}\right)=0$, and $\bar{\psi}_{t}\left(x_{0}, t_{0}\right)=0$. 
Case $2^{\circ}$. Assume that $\chi \phi\left(x_{0}, t_{0}\right)=1, x_{0} \in \partial \phi\left(t_{0}\right)$, and $D \psi\left(x_{0}, t_{0}\right) \neq 0$. The key observation is the following: Since $\phi$ is a barrier for $\mathcal{F}_{v}$ and evolution of a planar curve with velocity $v$ remains in the same plane we find that for an arbitrary plane $P, \underline{\phi}$ is a barrier for $\mathcal{F}_{\left.v\right|_{P}}^{=}$, the set of motions by velocity $v$ in the plane $P$.

That is, we claim that $\varrho:=\underline{\phi} \cap P \in \mathcal{B}\left(\mathcal{F}_{\left.v\right|_{P}}\right)$. This requires a proof since $\mathcal{F}_{v}$ contains only evolutions of curves, while $\mathcal{F}_{v \mid P}^{=}$contains evolutions of curves together with their interiors. The constructions required are simple and so we leave the proof to the reader. Lemma 6.1 now implies that $\varrho \in \mathcal{B}\left(\mathcal{F}_{\left.v\right|_{P}}^{<}\right)$. Therefore Theorem 5.1 in [5] shows that $(\chi \varrho)^{*}$ is a subsolution of the level-set equation corresponding to evolution of curves in the plane $P$ by velocity $v$ :

$$
w_{t}+F_{v}\left(D^{2} w, D w\right)=0 \quad \text { on } P \times[0, \infty) .
$$

Before we say what $F_{v}$ is, for a given symmetric matrix $X$ such that if $y \perp P$ then $X y=0$, and a nonzero vector $q \in P$, we denote by $\lambda$ the eigenvalue of $\frac{1}{|q|}(I-\hat{q} \otimes \hat{q}) X(I-\hat{q} \otimes \hat{q})$ that corresponds to an eigenvector in $P$ orthogonal to $q$. Then $F_{v}(X, q)=-|q| \operatorname{sign}(\lambda) v(|\lambda|,-\operatorname{sign}(\lambda) \hat{q}, \tilde{t})$, where $\tilde{t}$ is a unit vector in the plane $P$ orthogonal to $q$. The function $F_{v}$ has this (unusually complicated) form, because $v$ was defined as the velocity along the normal vector to the curve, while when applying the level-set approach (in the plane $P$ ), the outward normal velocity is needed.

Note that we know that $\chi \varrho=(\chi \varrho)^{*}$. Let $p:=D \widehat{\psi\left(x_{0}, t_{0}\right)}$.

Subcase (i). Assume that all eigenvalues of $\frac{1}{\left|D \psi\left(x_{0}, t_{0}\right)\right|}(I-p \otimes p) D^{2} \psi\left(x_{0}, t_{0}\right)(I-p \otimes p)$ that correspond to eigenvectors orthogonal to $p$ are nonnegative. Denote the eigenvalues by $k_{1}, \ldots, k_{n-1}$ and let $n$ be a unit vector such that $n \cdot p<0$. Let $t$ be a unit vector orthogonal to both $n$ and $p$, and let $\bar{k}$ be as before, $\bar{k}:=\sum_{i=1}^{n-1} k_{i}\left(l_{i} \cdot t\right)^{2}$, where for $i=1, \ldots, n-1, l_{i}$ is a unit eigenvector corresponding to $k_{i}$. Let $P:=\operatorname{span}\{n, t\}$, and let $\varrho$ be as above. Let $\psi_{P}$ be the restriction of $\psi$ to $P$. Note that $\left(x_{0}, t_{0}\right) \in \partial \varrho\left(t_{0}\right)$ and

$$
\begin{gathered}
D \psi_{P}\left(x_{0}, t_{0}\right)=D \psi\left(x_{0}, t_{0}\right) \cdot n n=\left|D \psi\left(x_{0}, t_{0}\right)\right| p \cdot n n, \\
\lambda\left(D^{2} \psi_{P}\left(x_{0}, t_{0}\right), D \psi_{P}\left(x_{0}, t_{0}\right)\right)=\frac{\bar{k}}{|n \cdot p|} .
\end{gathered}
$$

Since $\chi \varrho$ is a subsolution of the level-set equation,

$$
\begin{gathered}
\psi_{t}\left(x_{0}, t_{0}\right)+F_{v}\left(D^{2} \psi_{P}\left(x_{0}, t_{0}\right), D \psi_{P}\left(x_{0}, t_{0}\right)\right) \leqslant 0, \\
\psi_{t}\left(x_{0}, t_{0}\right)+\left|D \psi\left(x_{0}, t_{0}\right)\right| p \cdot n v\left(-\frac{\bar{k}}{n \cdot p}, n, t\right) \leqslant 0 .
\end{gathered}
$$

Therefore

$$
\psi_{t}\left(x_{0}, t_{0}\right)+\left|D \psi\left(x_{0}, t_{0}\right)\right| \sup _{\substack{n \in p^{-} \\ t \in n^{\perp} \cap p^{\perp}}} n \cdot p v\left(\frac{-\bar{k}}{n \cdot p}, n, t\right) \leqslant 0 .
$$

Hence, by definition of $V[v]$,

$$
\begin{gathered}
\psi_{t}\left(x_{0}, t_{0}\right)+\left|D \psi\left(x_{0}, t_{0}\right)\right| V[v]\left(\frac{(I-p \otimes p) D^{2} \psi\left(x_{0}, t_{0}\right)(I-p \otimes p)}{\left|D \psi\left(x_{0}, t_{0}\right)\right|}, p\right) \leqslant 0, \\
\psi_{t}\left(x_{0}, t_{0}\right)+F\left(D^{2} \psi\left(x_{0}, t_{0}\right), D \psi\left(x_{0}, t_{0}\right) \leqslant 0 .\right.
\end{gathered}
$$


Subcase (ii). Assume that there exists a negative eigenvalue of $(I-p \otimes p) D^{2} \psi\left(x_{0}, t_{0}\right)(I-p \otimes p)$. Let $p$ be as above. Let $n$ be a unit vector such that $n \cdot p>0$, and $t$ and $P$ as above. If $n$ and $t$ were such that $\bar{k}<0$ (note that there exist such $n$ and $t$ ) then since $\chi \varrho$ is a subsolution of the level-set equation

$$
\psi_{t}\left(x_{0}, t_{0}\right)+\left|D \psi\left(x_{0}, t_{0}\right)\right| p \cdot n v\left(-\frac{\bar{k}}{n \cdot p}, n, t\right) \leqslant 0 .
$$

Therefore

$$
\psi_{t}\left(x_{0}, t_{0}\right)+\left|D \psi\left(x_{0}, t_{0}\right)\right| \sup _{\substack{n \in p^{-} \\ t \in n^{\perp} \cap p^{\perp} \\ k}} n \cdot p v\left(\frac{-\bar{k}}{n \cdot p}, n, t\right) \leqslant 0 .
$$

Hence

$$
\psi_{t}\left(x_{0}, t_{0}\right)+\left|D \psi\left(x_{0}, t_{0}\right)\right| F\left(D^{2} \psi\left(x_{0}, t_{0}\right), p\right) \leqslant 0 .
$$

Case $3^{\circ}$. Assume that $\chi \underline{\phi}\left(x_{0}, t_{0}\right)=1$ and $x_{0} \notin \partial \underline{\phi}\left(t_{0}\right)$. Then $D \psi\left(x_{0}, t_{0}\right)=0$. We can assume that $\psi\left(x_{0}, t_{0}\right)=0$. Since $\bar{\psi}$ is admissible, $D^{2} \psi\left(x_{0}, t_{0}\right)=0$. Suppose that $\psi_{t}\left(x_{0}, t_{0}\right)>0$. Consider $(x, t)$ such that $t<t_{0}$ and $\left|x-x_{0}\right|^{2}<4(C+1)\left|t-t_{0}\right|$. Then

$$
\psi(x, t)=\psi_{t}\left(x_{0}, t_{0}\right)\left(t-t_{0}\right)+o\left(\left|t-t_{0}\right|\right) .
$$

Therefore there exists $\delta>0$, small, such that if $t \in\left(t_{0}-\delta, t_{0}\right)$ and $\left|x-x_{0}\right|^{2}<4(C+1)\left|t-t_{0}\right|$ then $\psi(x, t)<0$, which implies that $\chi \phi(x, t)=0$. Let $\gamma$ be a circle of radius $\sqrt{2(C+1) \delta}$, centered at $x_{0}$, in an arbitrary plane, $P$, in $\mathbb{R}^{n}$. Since on the circle, $\left|x-x_{0}\right|^{2}=2(C+1) \delta, \gamma$ is in the set $\phi\left(t_{0}-\delta\right)$. Let $\left\{\Gamma_{t}\right\}_{t \in\left[t_{0}-\delta, t_{0}\right]}$ be the evolution of $\gamma$ by $C$ times the curvature, and $\Omega_{t}$ the open set $\Gamma_{t}$ encloses. Note that $\Gamma_{t_{0}}$ is the circle of radius $\sqrt{\delta}$ centered at $x_{0}$. As in case $2^{\circ}, \phi \cap P \in \mathcal{B}\left(\mathcal{F}_{\left.v\right|_{P}}^{<}\right)$. Therefore, since $\left\{\left(\Omega_{t}, \Gamma_{t}\right)\right\}_{t \in\left[t_{0}-\delta, t_{0}\right]}$ is a strict subflow by velocity $\left.v\right|_{P}, \Omega_{t_{0}} \cup \Gamma_{t_{0}} \bar{\subset} \underline{\phi}\left(t_{0}\right)$. Therefore $x_{0} \in \phi\left(t_{0}\right)$. Contradiction. Therefore $\psi_{t}\left(x_{0}, t_{0}\right) \leqslant 0$.

Case $4^{\circ}$. Assume that $\chi \phi\left(x_{0}, t_{0}\right)=1, x_{0} \in \partial \phi\left(t_{0}\right)$, and $D \psi\left(x_{0}, t_{0}\right)=0$. The argument is analogous to the one in case $3^{\circ}$.

If velocity $v$ is not admissible, $V[v]$ can be infinite, which prevents us from proving the statement of the previous theorem for such velocities. Instead we prove the following:

PROPOSITION 6.6 Let $v(k, n, t)$ be a nonnegative velocity such that for every smooth curve contained in a 2-dimensional plane in $\mathbb{R}^{n}$ there exists a short time classical evolution by velocity $v$, and $V$ be a (continuous) velocity such that $V \leqslant V[v]$. Then, for a bounded set $E$,

$$
\mathcal{M}^{*}\left(E, \mathcal{F}_{V}^{\leqslant}\right) \subseteq \mathcal{M}^{*}\left(E, \mathcal{F}_{v}\right)
$$

REMARK. Recall that by Lemma 6.3. $\mathcal{M}^{*}\left(E, \mathcal{F}_{v}\right) \subseteq \mathcal{M}^{*}\left(E, \mathcal{F}_{V[v]}^{\leqslant}\right)$. It is not known whether for the velocities above $\mathcal{M}^{*}\left(E, \mathcal{F}_{v}\right)=\mathcal{M}^{*}\left(E, \mathcal{F}_{V[v]}^{\leqslant}\right)$if $V[v]$ is not continuous.

Proof. The proof of this proposition is analogous to the proof of (the appropriate statement in) Theorem 6.5. However, since we have no information on growth of $v$ any more, there are some new difficulties. Let $\phi$ and $\psi$ be as in the proof of the theorem (with $V[v]$ replaced by $V$ ). Proofs of cases $1^{\circ}$ and $2^{\circ}$ are analogous to the ones in the theorem. Let us consider: 
Case $3^{\circ}$. Assume that $\chi \phi\left(x_{0}, t_{0}\right)=1$ and $x_{0} \notin \partial \phi\left(t_{0}\right)$. Then $D \psi\left(x_{0}, t_{0}\right)=0$. Let $M \in$ $C^{\infty}([0, \infty))$ be such that $\overline{M^{\prime}}>0$ and $M(k)>\max \{v(\tilde{k}, \bar{n}, t): \tilde{k} \in[0, k],|n|=|t|=1, n \cdot t=0\}$. Let

$$
t(r):=\int_{0}^{r} \frac{d s}{M(1 / s)}, \quad c(r):=(t(r))^{2} .
$$

Using Lemma 7.2 we can assume that $\psi \in \mathcal{A}_{c}(F)$ and $\psi\left(x_{0}, t_{0}\right)=0$. Therefore for some $f \in$ $\mathcal{G}_{c}(F)$ and $\omega \in C^{1}([0, \infty),[0, \infty))$ such that $\lim _{r \rightarrow 0^{+}} \omega(r) / r=0, \omega^{\prime}(0)=0$ and $\delta>0$, and for all $(x, t) \in B\left(\left(x_{0}, t_{0}\right), \delta\right)$,

$$
\left|\psi(x, t)-\psi_{t}\left(x_{0}, t_{0}\right)\left(t-t_{0}\right)\right| \leqslant f\left(\left|x-x_{0}\right|\right)+\omega\left(\left|t-t_{0}\right|\right) .
$$

Let $a:=\psi_{t}\left(x_{0}, t_{0}\right)>0$. Consider $(x, t)$ such that $t\left(\left|x-x_{0}\right|\right) \leqslant 3\left|t-t_{0}\right|$ and $t<t_{0}$. Then

$$
\begin{aligned}
\psi(x, t) & \leqslant a\left(t-t_{0}\right)+f\left(\left|x-x_{0}\right|\right)+\omega\left(\left|t-t_{0}\right|\right) \\
& \leqslant-a\left|t-t_{0}\right|+t\left(\left|x-x_{0}\right|\right)^{2}+o\left(t-t_{0}\right) \\
& \leqslant-a\left|t-t_{0}\right|+o\left(t-t_{0}\right) .
\end{aligned}
$$

Therefore there exists $\delta_{1}>0$ such that if $t \in\left(t_{0}-\delta_{1}, t_{0}\right)$ and $t\left(\left|x-x_{0}\right|\right) \leqslant 3\left|t-t_{0}\right|$ then $\psi(x, t)<0$,

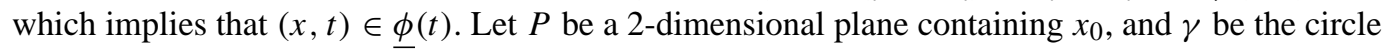
centered at $x_{0}$ with radius $t^{-1}\left(2 \delta_{1}\right)$. Consider the motion by velocity $v$ in the plane $P$. As in Theorem 6.5. one shows that $\underline{\phi} \cap P \in \mathcal{B}\left(\mathcal{F}_{\left.v\right|_{P}}^{<}\right)$. For $t \in\left[t_{0}-\delta_{1}, t_{0}+\delta_{1}\right)$ let $\Gamma_{t}$ be the circle in the same plane as $\gamma$, centered at $x_{0}$, with radius $r(t):=t^{-1}\left(t_{0}-t+\delta_{1}\right)$. Note that $r^{\prime}(t)=-M\left(\frac{1}{r\left(t_{0}-t\right)}\right)$. Therefore the outward normal velocity of $\Gamma_{t}$ is by definition of $M$ less than $-v$. Hence $\Gamma_{t}$ is a strict subflow by velocity $\left.v\right|_{P}$.

Since $\underline{\phi} \cap P \in \mathcal{B}\left(\mathcal{F}_{v \mid p}^{<}\right), \Gamma_{t} \cup \Omega_{t} \subset \underline{\phi}(t)$, where $\Omega_{t}$ is the interior of the circle $\Gamma_{t}$. Therefore $x_{0} \in \phi(t)$. Contradiction. Therefore $\psi_{t}\left(x_{0}, t_{0}\right) \leqslant 0$.

Proof of case $4^{\circ}$ is analogous to the proof of case $3^{\circ}$.

In Theorem 6.5 we have shown that for admissible velocities barriers are essentially equivalent to surrounding. The proposition above can be used to show that minimal barriers do not capture the motion by many of the velocities $v$ that are not admissible. Let us just illustrate how that can be done on an example:

EXAMPLE. Let $v(k, n, t)=k^{\alpha}$ with $\alpha>1$. Then

$$
V[v]\left(R \operatorname{diag}\left(k_{1}, \ldots, k_{n-1}, 0\right) R^{-1}, p\right)= \begin{cases}-\left(\min _{i=1, \ldots, n-1} k_{i}\right)^{\alpha} & \text { if } k_{i} \geqslant 0 \text { for all } i, \\ \infty & \text { otherwise. }\end{cases}
$$

Let $\Gamma_{0}$ be any circle of radius 1 in $\mathbb{R}^{n}$. Let $\Gamma_{t}$ be the evolution of the circle by constant velocity $v_{0}:=2$ for time $t<T:=\frac{1}{2}\left(1-2^{-1 / \alpha}\right)$. Let

$$
V\left(R \operatorname{diag}\left(k_{1}, \ldots, k_{n-1}, 0\right) R^{-1}, p\right):= \begin{cases}-\left(\min _{i=1, \ldots, n-1} k_{i}\right)^{\alpha} & \text { if } k_{i} \geqslant 0 \text { for all } i \\ -4\left(\min _{i=1, \ldots, n-1} k_{i}\right) & \text { otherwise. }\end{cases}
$$

Note that $V \leqslant V[v]$. We claim that $\Gamma_{t} \subset \mathcal{M}^{*}\left(\Gamma_{0}, \mathcal{F}_{v}\right)(t)$. Since $\left\{\Gamma_{t}\right\}_{t \in[0, T]}$ is not the evolution of $\Gamma_{0}$ with velocity $v$ this shows that the minimal barrier contains more than just the evolution of 
$\Gamma_{0}$ by velocity $v$. To prove the claim, by Proposition 6.6, it is enough to show that for any $\rho>0$ small enough, $\Gamma_{t} \subseteq \mathcal{M}\left(\Gamma_{0}+\rho, \mathcal{F}_{V}^{\leqslant}\right)(t)$. To see this, it is enough to show that if $\phi \in \mathcal{B}\left(\mathcal{F}_{V}^{\leqslant}\right)$and $\Gamma_{0}+\rho \subseteq \phi(0)$ then $\Gamma_{t} \subset \phi(t)$ for all $t \in(0, T)$.

So let $\sigma$ be as in Lemma3.2 $\rho \in(0, \sigma), \Omega_{0}:=\Gamma_{0}+\rho / 2$, and $\Upsilon_{0}:=\partial \Omega_{0}$. Let $\left\{\left(\Omega_{t}, \Upsilon_{t}\right)\right\}_{t \in[0, T]}$ be the generalized evolution of $\left(\Omega_{0}, \Upsilon_{0}\right)$ by velocity $V$. By Theorem 5.1 in [5], $\chi \phi$ is a supersolution of the level-set equation, and hence by comparison, $\Omega_{t} \cup \Upsilon_{t} \subset \phi(t)$ for all $t$. So it suffices to show that $\Gamma_{t} \subseteq \Omega_{t}$ for $t \in(0, T)$. Let $d(z, t)$ be the distance from $\bar{z}$ to $\Gamma_{t}$. Let $t \in[0, T)$, and $z$ such that $0<s:=d(z, t)<\sigma$. Let $x$ be the point on $\Gamma_{t}$ closest to $z, p:=\widehat{z-x}$, and $n$ the unit normal vector to $\Gamma_{t}$ at $x$. Note that at time $t, \Gamma_{t}$ is a circle of radius $1-2 t$ with curvature $k=1 /(1-2 t)$. Then 3.5 implies

$$
\begin{aligned}
d_{t}(z, t)+F\left(D^{2} d(z, t), D d(z, t)\right) & =-2 n \cdot p+ \begin{cases}-\frac{(k|n \cdot p|)^{\alpha}}{(1+s k|n \cdot p|)^{\alpha}} & \text { if } n \in p^{-}, \\
\frac{4 k n \cdot p}{1-s k n \cdot p} & \text { otherwise }\end{cases} \\
& \geqslant 0 .
\end{aligned}
$$

By comparison, as in Lemma 4.6 this inequality implies that $\Gamma_{t} \subset \Omega_{t}$ for $t \in[0, T)$. Therefore $\Gamma_{t} \subset \mathcal{M}^{*}\left(\Gamma_{0}, \mathcal{F}_{v}\right)(t)$.

\subsection{Simple proof of the original De Giorgi's conjecture}

The arguments above have dealt only with the motion of curves along the normal vector. To show that the techniques we used can be extended to higher dimensions let us now prove the De Giorgi's conjecture regarding the motion of $k$-dimensional manifolds in $\mathbb{R}^{n}$ by mean curvature. As mentioned in the introduction, the conjecture was originally proven by Bellettini and Novaga in [6].

Proposition 6.7 Let $v(S, T)$ be ( $k$ times) the mean curvature vector and $V(D n, n)$ be (minus) the sum of the $k$ smallest eigenvalues of $D n$ that correspond to eigenvectors that are orthogonal to $n$. Then, for a bounded set $E$,

$$
\mathcal{M}^{*}\left(E, \mathcal{F}_{v}\right)(t)=\mathcal{M}^{*}\left(E, \mathcal{F}_{V}^{\leqslant}\right)(t)=\{x: u(x, t)=0\},
$$

where $u$ is the unique viscosity solution of the level-set equation:

$$
\begin{aligned}
& u_{t}+F\left(D^{2} u, D u\right)=0, \\
& u(x, 0)=\operatorname{dist}_{ \pm}(x, E) .
\end{aligned}
$$

Proof. As before the second equality follows from Corollary 6.1 in [5].

To prove $\mathcal{M}^{*}\left(E, \mathcal{F}_{v}\right)(t) \subseteq \mathcal{M}^{*}\left(E, \mathcal{F}_{V}^{\leqslant}\right)(t)$, recall that $v$ and $V$ satisfy condition $(\diamond)$. The proof is then analogous to the proof of Lemma 6.3 .

Let us now prove the opposite inclusion: $\mathcal{M}^{*}\left(E, \mathcal{F}_{V}^{\lessgtr}\right)(t) \subseteq \mathcal{M}^{*}\left(E, \mathcal{F}_{v}\right)(t)$. As in Theorem 6.5 it is enough to prove that if $\phi(t)$ is a barrier for $\mathcal{F}_{v}$ then $\chi \phi(t)$ is a viscosity subsolution of the level-set equation $u_{t}+F\left(D^{2} u, D u\right)=0$. Note that $F_{*}(0,0)=F^{*}(0,0)=0$. Let $\psi$ be a smooth test function such that $\chi \underline{\varphi}-\psi$ has a maximum at $\left(x_{0}, t_{0}\right)$. Cases $1^{\circ}, 3^{\circ}$, and $4^{\circ}$ are the same as in the theorem. So let us consider

Case $2^{\circ}$. Assume that $\chi \phi\left(x_{0}, t_{0}\right)=1, x_{0} \in \partial \phi\left(t_{0}\right)$, and $D \psi\left(x_{0}, t_{0}\right) \neq 0$. Observe that if a $k$ dimensional manifold is contained in a $k+1$-dimensional plane $P$, then its evolution by mean 
curvature remains in the same plane. As in the proof of Theorem 6.5 one shows that $\varrho:=\phi \cap$ $P \in \mathcal{B}\left(\mathcal{F}_{v \mid p}^{<}\right)$. Let $p:=D \widehat{\psi\left(x_{0}, t_{0}\right)}$ and $\lambda_{1}, \ldots, \lambda_{k}$ be the $k$ smallest eigenvalues of $(I-\bar{p} \otimes$ p) $D^{2} \psi\left(x_{0}, t_{0}\right)(I-p \otimes p)$ with eigenvectors orthogonal to $p$. Let $l_{1}, \ldots, l_{k}$ be a set of corresponding unit eigenvectors and let $P:=\operatorname{span}\left\{l_{1}, \ldots, l_{k}, p\right\}$. Since $\varrho \in \mathcal{B}\left(\mathcal{F}_{\left.v\right|_{p}}^{<}\right),(\chi \varrho)^{*}$ is by Theorem 5.1 of [5] a subsolution of the level-set equation of the motion by mean curvature in $P, w_{t}-\operatorname{trace}(I-\widehat{D w} \otimes$ $\widehat{D w}) D^{2} w(I-\widehat{D w} \otimes \widehat{D w})=0$. Recall that $\chi \varrho=(\chi \varrho)^{*}$ and $\chi \varrho-\left.\psi\right|_{P}$ has a maximum at $\left(x_{0}, t_{0}\right)$. We also have $D \psi_{P}\left(x_{0}, t_{0}\right)=D \psi\left(x_{0}, t_{0}\right)$ and trace $(I-p \otimes p) D^{2} \psi_{P}\left(x_{0}, t_{0}\right)(I-p \otimes p)=\lambda_{1}+\cdots+\lambda_{k}$. Since $\chi \varrho$ is a subsolution,

$$
0 \geqslant \frac{\partial \psi_{P}}{\partial t}\left(x_{0}, t_{0}\right)-\operatorname{trace}(I-p \otimes p) D^{2} \psi_{P}\left(x_{0}, t_{0}\right)(I-p \otimes p)=\frac{\partial \psi}{\partial t}-\left(\lambda_{1}+\cdots+\lambda_{k}\right)
$$

\section{Appendix}

Consider the level-set equation

$$
u_{t}+F\left(D^{2} u, D u\right)=0 \quad \text { in } \mathbb{R}^{n} \times[0, T) .
$$

The functions $F$ that we consider have the following properties:

(F1) $F \in C\left(\operatorname{Sym}(n) \times\left(\mathbb{R}^{n} \backslash\{0\}\right)\right)$.

(F2) $F$ is degenerate elliptic, that is, for all symmetric matrices $X$ and $Y$ and all nonzero vectors $p$,

$$
\text { if } X \leqslant Y \text { then } F(X, p) \geqslant F(Y, p) .
$$

(F3) $F$ is geometric, that is, for all $\lambda>0, \mu \in \mathbb{R}$, all symmetric matrices $X$, and nonzero vectors $p$,

$$
F(\lambda X+\mu p \otimes p, \lambda p)=\lambda F(X, p) .
$$

To define a viscosity solution for the level-set equation in this generality, we follow Ishii and Souganidis. Given a function $F$ that satisfies $(\mathrm{F} 1)-(\mathrm{F} 3)$ we let $\mathcal{G}(F)$ be the set of all functions $g \in C^{2}([0, \infty),[0, \infty))$ such that $g(0)=g^{\prime}(0)=g^{\prime \prime}(0)=0, g^{\prime \prime}(r)>0$ if $r>0$, and

$$
\lim _{p \rightarrow 0} \frac{g^{\prime}(|p|)}{|p|} F(I, p)=\lim _{p \rightarrow 0} \frac{g^{\prime}(|p|)}{|p|} F(-I, p)=0 .
$$

It was shown in [14] that $\mathcal{G}(F) \neq \varnothing$.

We say that a function $\varphi \in C^{2}\left(\mathbb{R}^{n} \times(0, T)\right)$ is admissible if for all points $\left(x_{0}, t_{0}\right)$ such that $D \varphi\left(x_{0}, t_{0}\right)=0$, there exists a constant $\delta>0$ and functions $g \in \mathcal{G}(F)$ and $\omega \in C([0, \infty),[0, \infty))$ satisfying $\lim _{r \rightarrow 0^{+}} \omega(r) / r=0$ such that, for all $(x, t)$ in $B\left(\left(x_{0}, t_{0}\right), \delta\right)$,

$$
\left|\varphi(x, t)-\varphi\left(x_{0}, t_{0}\right)-\varphi_{t}\left(x_{0}, t_{0}\right)\left(t-t_{0}\right)\right| \leqslant g\left(\left|x-x_{0}\right|\right)+\omega\left(\left|t-t_{0}\right|\right) .
$$

Let $\mathcal{A}(F)$ be the set of all admissible functions.

Definition 7.1 An upper semicontinuous function $u: \mathbb{R}^{n} \times(0, T) \rightarrow[-\infty, \infty)$ is a viscosity subsolution of the equation 7.1 if for all $\varphi \in C^{2}\left(\mathbb{R}^{n} \times(0, T)\right)$ and all points $(x, t)$ such that $u-\varphi$ has a local maximum at $(x, t)$ :

- If $D \varphi(x, t) \neq 0$ then $\varphi_{t}(x, t)+F\left(D^{2} \varphi(x, t), D \varphi(x, t)\right) \leqslant 0$.

- If $D \varphi(x, t)=0$ and $\varphi \in \mathcal{A}(F)$ then $\varphi_{t}(x, t) \leqslant 0$.

Viscosity supersolutions are defined analogously. 
The following modification of the definition has been used in the section on barriers of De Giorgi. Given a function $c \in C^{2}\left(\mathbb{R}^{+}, \mathbb{R}^{+}\right)$we define $\mathcal{G}_{c}(F)$ to be the functions in $\mathcal{G}(F)$ that are less than or equal to $c$. Note that $\mathcal{G}_{c}(F) \neq \emptyset$. We define $\mathcal{A}_{c}(F)$ in the obvious way.

Following ideas from Proposition 2.2 in [2] and Proposition 1.3 in [14] we prove:

LEMMA 7.2 Let $u: \mathbb{R}^{n} \times(0, T) \rightarrow[-\infty, \infty)$ be an upper semicontinuous function. For $u$ to be a viscosity subsolution of the equation $(7.1)$ it is sufficient that for all $\varphi \in C^{2}\left(\mathbb{R}^{n} \times(0, T)\right)$ and all points $(x, t)$ such that $u-\varphi$ has a local maximum at $(x, t)$ :

- If $D \varphi(x, t) \neq 0$ then $\varphi_{t}(x, t)+F\left(D^{2} \varphi(x, t), D \varphi(x, t)\right) \leqslant 0$.

- If $D \varphi(x, t)=0$ and $\varphi \in \mathcal{A}_{c}(F)$ then $\varphi_{t}(x, t) \leqslant 0$.

An analogous claim holds for viscosity supersolutions.

Proof. Let $u$ be an upper semicontinuous function that satisfies the condition above. To show that $u$ is a viscosity subsolution of $(7.1)$ it is enough to prove that for a function $\varphi \in \mathcal{A}(F)$ and a point $\left(x_{0}, t_{0}\right)$ such that $u-\varphi$ has a local maximum at $\left(x_{0}, t_{0}\right)$ and $D \varphi\left(x_{0}, t_{0}\right)=0$ we have $\varphi_{t}\left(x_{0}, t_{0}\right) \leqslant 0$. So let $\varphi$ and $\left(x_{0}, t_{0}\right)$ be as described. Since $\varphi$ is admissible there exist $f \in \mathcal{G}(F), \omega$ with $\lim _{r \rightarrow 0^{+}} \omega(r) / r=0$, and $\delta>0$ such that, for all $(x, t)$ in $B\left(\left(x_{0}, t_{0}\right), \delta\right)$,

$$
\left|\varphi(x, t)-\varphi\left(x_{0}, t_{0}\right)-\varphi_{t}\left(x_{0}, t_{0}\right)\left(t-t_{0}\right)\right| \leqslant f\left(\left|x-x_{0}\right|\right)+\omega\left(\left|t-t_{0}\right|\right) .
$$

Without loss of generality we can assume that $\omega \in C^{1}(\mathbb{R}), \omega(0)=0, \omega$ is even, and $\omega(r)>0$ if $r>0$. Let $\bar{\varphi}(x, t):=\varphi_{t}\left(x_{0}, t_{0}\right)\left(t-t_{0}\right)+f\left(\left|x-x_{0}\right|\right)+\omega\left(t-t_{0}\right)$. Note that $u-\bar{\varphi}$ has a local maximum at $\left(x_{0}, t_{0}\right)$. By perturbing $f$ and $\omega$ if necessary, we can assume that the maximum is strict. Let $g \in \mathcal{G}_{c}(F)$. For $\varepsilon>0$, consider the function

$$
w_{\varepsilon}(x, y, t):=u(x, t)-\bar{\varphi}(y, t)-g(|x-y|) / \varepsilon .
$$

Since $\left(x_{0}, t_{0}\right)$ is a strict local maximum of $u-\bar{\varphi}$ there exists a sequence of local maxima $\left(x_{\varepsilon}, y_{\varepsilon}, t_{\varepsilon}\right)$ of $w_{\varepsilon}$ such that $x_{\varepsilon} \rightarrow x_{0}, y_{\varepsilon} \rightarrow x_{0}, t_{\varepsilon} \rightarrow t_{0}$, and $w_{\varepsilon} \rightarrow u\left(x_{0}, t_{0}\right)$ as $\varepsilon \rightarrow 0$. We need to consider two cases:

Case $1^{\circ}$. Assume that $D \bar{\varphi}\left(y_{\varepsilon}, t_{\varepsilon}\right)=0$ for some $\varepsilon$. Then $x_{\varepsilon}=y_{\varepsilon}=x_{0}$ and $t_{\varepsilon}=t_{0}$. Let $\psi(x, t):=$ $\bar{\varphi}\left(x_{0}, t\right)+g\left(\left|x-x_{0}\right|\right) / \varepsilon$. Note that $u-\psi$ has a local maximum at $\left(x_{0}, t_{0}\right)$ and $\psi \in \mathcal{A}_{c}(F)$. Therefore by assumption $\psi_{t}\left(x_{0}, t_{0}\right)=0$. But $\psi_{t}\left(x_{0}, t_{0}\right)=\varphi_{t}\left(x_{0}, t_{0}\right)$.

Case $2^{\circ}$. Assume that $D \bar{\varphi}\left(y_{\varepsilon}, t_{\varepsilon}\right) \neq 0$ for all $\varepsilon$. Let

$$
\psi_{\varepsilon}(x, t):=\bar{\varphi}\left(x-\left(x_{\varepsilon}-y_{\varepsilon}\right), t\right)+g\left(\left|x_{\varepsilon}-y_{\varepsilon}\right|\right) / \varepsilon .
$$

Note that $u-\psi_{\varepsilon}$ has a local maximum at $\left(x_{\varepsilon}, t_{\varepsilon}\right)$ and

$$
\begin{gathered}
\frac{\partial \psi_{\varepsilon}}{\partial t}\left(x_{\varepsilon}, t_{\varepsilon}\right)=\bar{\varphi}_{t}\left(y_{\varepsilon}, t_{\varepsilon}\right), \quad D \psi_{\varepsilon}\left(x_{\varepsilon}, t_{\varepsilon}\right)=f^{\prime}\left(\left|p_{\varepsilon}\right|\right) \frac{p_{\varepsilon}}{\left|p_{\varepsilon}\right|}, \\
D^{2} \psi_{\varepsilon}\left(x_{\varepsilon}, t_{\varepsilon}\right)=f^{\prime \prime}\left(\left|p_{\varepsilon}\right|\right)\left(\frac{p_{\varepsilon}}{\left|p_{\varepsilon}\right|} \otimes \frac{p_{\varepsilon}}{\left|p_{\varepsilon}\right|}\right)+\frac{f^{\prime}\left(\left|p_{\varepsilon}\right|\right)}{\left|p_{\varepsilon}\right|}\left(I-\frac{p_{\varepsilon}}{\left|p_{\varepsilon}\right|} \otimes \frac{p_{\varepsilon}}{\left|p_{\varepsilon}\right|}\right),
\end{gathered}
$$

where $p_{\varepsilon}:=y_{\varepsilon}-x_{0}$. Therefore

$$
\bar{\varphi}_{t}\left(y_{\varepsilon}, t_{\varepsilon}\right)+\omega^{\prime}\left(t_{\varepsilon}-t_{0}\right)+F\left(\frac{f^{\prime}\left(\left|p_{\varepsilon}\right|\right)}{\left|p_{\varepsilon}\right|} I, f^{\prime}\left(\left|p_{\varepsilon}\right|\right) \frac{p_{\varepsilon}}{\left|p_{\varepsilon}\right|}\right) \leqslant 0 .
$$

Taking the limit as $\varepsilon \rightarrow 0$, and using the geometricity of $F$ and the fact that $f \in \mathcal{G}(F)$, we obtain $\varphi_{t}\left(x_{0}, t_{0}\right) \leqslant 0$. 


\section{REFERENCES}

1. Ambrosio, L. \& Soner, H. M. Level-set approach to mean curvature flow in arbitrary codimension. J. Differential Geom. 43 (1996), 693-737. Zbl 0868.35046 MR 97k:58047

2. BARles, G. \& Georgelin, C. A simple proof of convergence for an approximation scheme for computing motions by mean curvature. SIAM J. Numer. Anal. 32 (1995), 484-500. Zbl 0831.65138 MR 96c:65140

3. Barles, G., Soner, H. M., \& Souganidis, P. E. Front propagation and phase field theory. SiAM J. Control Optim. 31 (1993), 439-469. Zbl 0785.35049 MR 94c:35005

4. Bellettini, G. \& Novaga, M. Minimal barriers for geometric evolutions. J. Differential Equations 139 (1997), 76-103. Zbl 0882.35028 MR 98j:35083

5. Bellettini, G. \& Novaga, M. Comparison results between minimal barriers and viscosity solutions for geometric evolutions. Ann. Scoula Norm. Sup. Pisa Cl. Sci. 26 (1998), 97-131. Zbl 0904.35041 MR 99i:35081

6. Bellettini, G. \& Novaga, M. A result on motion by mean curvature in arbitrary codimension. Diff. Geom. Appl. 11 (1999), 205-220. Zbl 0959.35085 MR 2001c:53092

7. DO CARMO, M. P. Riemannian Geometry. Birkhäuser (1992). Zbl 0752.53001 MR 92i:53001

8. Chen, Y. G., Giga, Y., \& Goto, S. Uniqueness and existence of viscosity solutions of generalized mean curvature flow equations. J. Differential Geom. 33 (1991), 749-786. Zbl 0696.35087 MR 93a:35093

9. Crandall, M. G., Ishit, H., \& LiOns, P. L. User's guide to viscosity solutions of second order partial differential equations. Bull. Amer. Math. Soc. 27 (1992), 1-67. Zbl 0755.35015 MR 92j:35050

10. De Giorgi, E. Barriers, boundaries, motion of manifolds. Conference held at Department of Mathematics, University of Pavia, March 18, 1994.

11. Evans, L. C. \& Spruck, J. Motion of level sets by mean curvature I. J. Differential Geom. 33 (1991), 635-681. Zbl 0726.53029 MR 92h:35097

12. Giga, Y. \& Goto, S. Geometric evolution of phase-boundaries. On the Evolution of Phase Boundaries. IMA Vol. Math. Appl. 43, Springer (1992), 51-65. Zbl 0771.35027 MR 94g:35226

13. HiRsch, M. Differential Topology. Springer (1976). Zbl 0356.57001 MR 96c:57001

14. IshiI, H. \& Souganidis, P. E. Generalized motion on noncompact hypersurfaces with velocities having arbitrary growth on the curvature tensor. Tohoku Math. J. 47 (1995), 227-250. Zbl 0837.35066 MR 96e:35069

15. Lions, P. L. \& Souganidis, P. E. Fully non-linear second-order degenerate elliptic equations with large zeroth-order coefficients. Indiana Univ. Math. J. 42 (1993), 1525-1543. Zbl 0791.35040 MR 95c:35085

16. Osher, S. \& Sethian, J. A. Fronts propagating with curvature dependent speed: algorithms based on Hamilton-Jacobi equations. J. Comput. Phys. 79 (1988), 12-49. Zbl 0659.65132 MR 89h:80012

17. SONER, H. M. Motion of a set by the curvature of its boundary. J. Differential Equations 101 (1993), 313-372. Zbl 0769.35070 MR 94b:5803 